\title{
Efficiency of shear-induced agglomeration of particulate suspensions subjected to bridging flocculation
}

\author{
Sushant Agarwal \\ West Virginia University
}

Follow this and additional works at: https://researchrepository.wvu.edu/etd

\section{Recommended Citation}

Agarwal, Sushant, "Efficiency of shear-induced agglomeration of particulate suspensions subjected to bridging flocculation" (2002). Graduate Theses, Dissertations, and Problem Reports. 2406.

https://researchrepository.wvu.edu/etd/2406

This Dissertation is protected by copyright and/or related rights. It has been brought to you by the The Research Repository @ WVU with permission from the rights-holder(s). You are free to use this Dissertation in any way that is permitted by the copyright and related rights legislation that applies to your use. For other uses you must obtain permission from the rights-holder(s) directly, unless additional rights are indicated by a Creative Commons license in the record and/ or on the work itself. This Dissertation has been accepted for inclusion in WVU Graduate Theses, Dissertations, and Problem Reports collection by an authorized administrator of The Research Repository @ WVU.

For more information, please contact researchrepository@mail.wvu.edu. 


\title{
Efficiency of Shear-Induced Agglomeration of Particulate
}

\author{
Suspensions Subjected to Bridging Flocculation
}

\author{
Sushant Agarwal
}

Dissertation submitted to College of Engineering and Mineral Resources at West Virginia University in partial fulfillment of the requirements of Doctor of Philosophy in

Chemical Engineering

\author{
Dr. Rakesh K. Gupta, Ph. D., Chair \\ Dr. John W. Zondlo, Ph. D. \\ Dr. Ray Y. K. Yang, Ph. D. \\ Dr. Donald D. Gray, Ph. D. \\ Dr. Ramesh M. Pisipati, Ph. D.
}

Department of Chemical Engineering

Morgantown, West Virginia

2002

Keywords: Orthokinetic Agglomeration, Flocculation, Polymer Bridging, Collision Efficiency, Agglomerate size 


\section{ABSTRACT \\ Efficiency of Shear-Induced Agglomeration of Particulate Suspensions Subjected to Bridging Flocculation}

\section{Sushant Agarwal}

This study examines the problem of shear-induced agglomeration of particles such as fillers and pigments in polymer solutions, where polymer molecules adsorb on the surface of particles and form a bridge between them causing agglomeration. The rate of agglomeration is usually obtained by multiplying the rate of collision of particles by the collision efficiency. In a laminar flow field, the collision frequency is readily given by Smoluchowski's expression. If agglomeration takes place by coagulation, the collision efficiency is well studied and the influences of hydrodynamic and colloidal forces are well understood. In case of polymer bridging, the collision efficiency is determined by probabilistic models based on fractional surface coverage of particles by the adsorbing polymer, neglecting the influence of hydrodynamic forces. A new model is required to determine the collision efficiency in case of shear-induced agglomeration of non-colloidal size particles by polymer bridging.

In this work, a new model is developed in terms of dimensionless groups and the fractional surface coverage. The dimensionless groups represent the relative magnitudes of the colloidal, hydrodynamic and steric forces. An expression for the optimum surface coverage needed to achieve the maximum collision efficiency is also obtained. In particular, a model is presented for the case where surface of the particles is completely covered by a polymer layer.

To validate this model, experimental collision efficiencies at various agglomeration conditions are determined by shearing a model suspension in a cone-and-plate device. The model suspension consisted of $4.9 \mu \mathrm{m}$ diameter spherical hollow glass beads dispersed in an aqueous glycerin solution; polyethylene oxide was used as the flocculant. Using experimental collision efficiencies and non-linear regression analysis, the parameters in the collision efficiency model were determined.

It was found that increasing the shear rate decreases the collision efficiency and it can be expressed as a power law. Increasing the molecular weight and concentration of flocculant also gives higher collision efficiency. Agglomerate growth and the equilibrium agglomerate size were also monitored under different flocculation conditions. It was found that the size to which agglomerates grow depends on the shear rate and this can be expressed as a power law as $\mathrm{d}_{\mathrm{f}, 95 \%} \propto \dot{\gamma}^{-0.258}$. In addition, agglomerate breakage studies show that the agglomerate breakage occurs by floc rupture mechanism rather than by the surface erosion of primary particles. 
Dedicated to my parents

(late) Mr. Shiv Kumar Gupta and

Mrs. Usha Agarwal 


\section{Acknowledgements}

I would like to use this opportunity to thank everybody without whose help this work would have not been possible.

First of all, I would like to express my deep gratitude to my adviser Dr. Rakesh K. Gupta. His advice, support and encouragement were invaluable and indispensable many a times. I am also thankful to him for exposing me to other aspects of polymer science and rheology. I continue to benefit from his advice and hopefully in the future as well.

I am also thankful to my committee members- Dr. John W. Zondlo, Dr. Ray Y. K. Yang, Dr. Donald D. Gray and Dr. Ramesh M. Pisipati for their help and advice.

I also acknowledge the help of Dr. Deepak Doraiswamy at Dupont for his advice all

through this project. I thank Dupont for providing financial support for the part of this research.

A special note of thanks to technician Mr. Jim Hall and office manager Ms. Linda Rogers for all their help on so many occasions.

Finally, my sincere thank to my family for all their support and encouragement over the years, without which none of this would be possible. I also thank all the friends that I have made here over the years for their friendship and support. 


\section{Table of Contents}

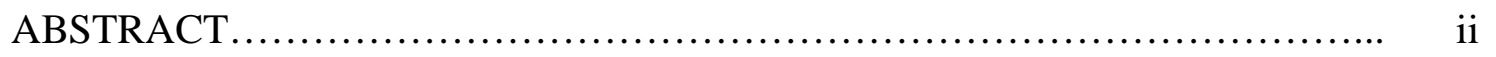

ACKNOWLEDGEMENTS ................................................ iv

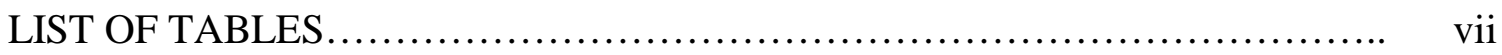

LIST OF FIGURES....................................................... viii

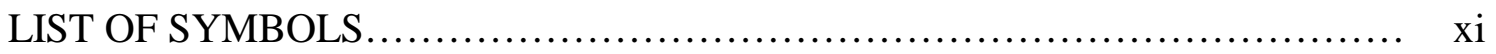



2. BACKGROUND OF AGGLOMERATION PROCESSES ........................ 5

2.1 Perikinetic Collision Frequency........................................ 5

2.2 Differential Sedimentation Frequency .................................... 7

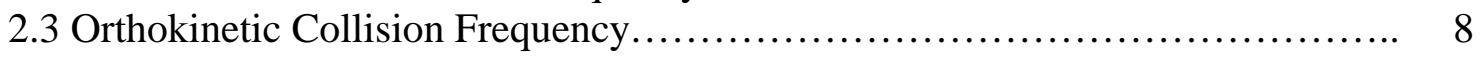

2.4 Perikinetic Collision Efficiency............................................ 11

2.5 Orthokinetic Collision Efficiency......................................... 15

2.6 Orthokinetic Rate of Agglomeration..................................... 21

3. REVIEW OF POLYMER BRIDGING FLOCCULATION ....................... 23

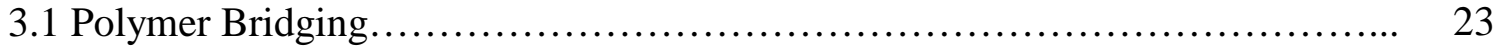

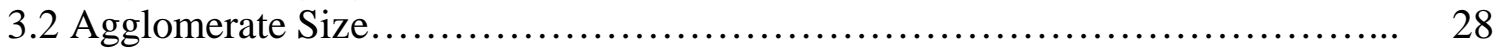

3.3 Parameters Governing Collision Efficiency and Agglomerate Size.............. 34

3.3.1 Effect of Solvent............................................... 34

3.3.2 Effect of Molecular Weight of Polymer............................... 36

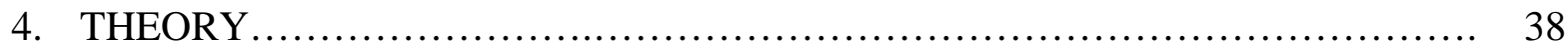

4.1 Development of Collision Efficiency Model................................ 38

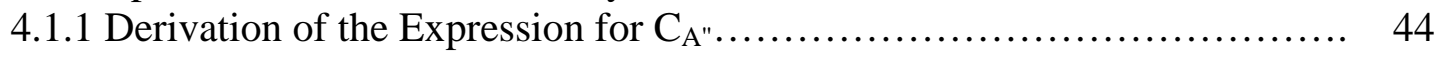

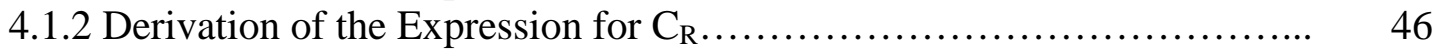

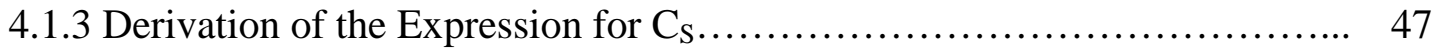

5. EXPERIMENTAL DETAILS................................................. 49

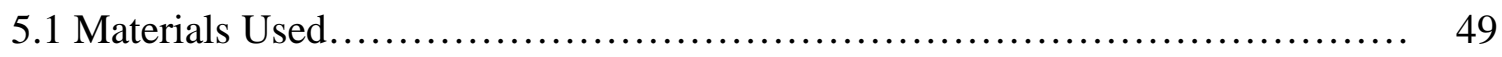

5.1.1 Solid Particles................................................... 49

5.1.2 Dispersion Medium................................................ 49

5.1 .3 Polymer Flocculant................................................. 51

5.2 Suspension Preparation.................................................. 52

5.3 Shearing of the Suspension.............................................. 53

5.4 Image Analysis........................................................ 55

5.5 Estimation of Flory-Huggins Parameter and Polymer Molecule Size............. $\quad 56$

5.6 Adsorption of PEO .................................................... 61

5.7 Estimation of Thickness of Adsorbed Polymer Layer.......................... 65 
6. RESULTS AND DISCUSSION_............................................. 67

6.1 Experimental Determination of Collision Efficiency......................... 68

6.2 Determination of Exponents Appearing in the Collision Efficiency Model........ 76

6.3 Effect of Polymer Concentration on Collision Efficiency......................... 84

6.4 Effect of Shear Rate on Collision Efficiency ................................ 87

6.5Effect of Molecular Weight of PEO on Collision Efficiency..................... 90

6.6Effect of Shear Rate on Agglomerate Size.................................. 90

6.7 Effect of Molecular Weight of PEO on Agglomerate Size...................... 97

6.8 Agglomerate Breakage ..................................................... 99

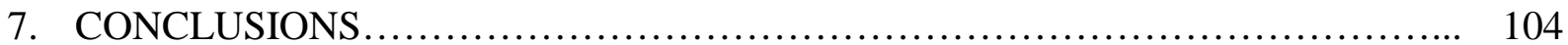

7.1 Collision Efficiency................................................... 104

7.2 Agglomerate Size................................................... 106

8. RECOMMENDATIONS FOR FUTURE WORK ............................. 108

REFERENCES................................................................ 111

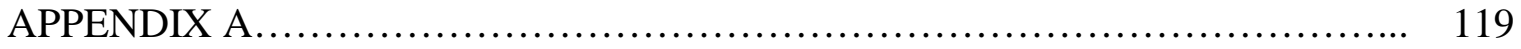



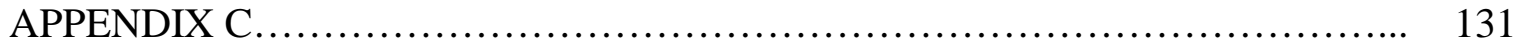




\section{List of Tables}

Table 5.1 Comparison of sizes of standard spherical particles 56

Table 5.2 Measured intrinsic viscosity at $\Theta$-conditions and the expansion factor $\quad 58$

Table 5.3 Size of PEO molecules in the solvent $\quad 60$

Table 5.4 Estimation of Flory-Huggins parameter 61

Table 5.5 Calculated values of $\mathrm{c}$ and $\delta \quad 66$

Table 6.1 Experimental values of collision efficiency 74

Table 6.2 Calculated values of $\mathrm{C}_{\mathrm{A}^{\prime \prime}} \quad 78$

Table 6.3 Calculated values of $\mathrm{C}_{\mathrm{R}^{\prime}} \quad 79$

Table 6.4 Calculated values of $\mathrm{C}_{\mathrm{S}} \quad 81$

Table 6.5 Data used for non-linear regression $\quad 82$

Table 6.6 Results of Regression Analysis $\quad 83$

Table 6.7 Comparison of predicted and Experimental values of collision

efficiency $\quad 84$ 


\section{List of Figures}

Figure 2.1. Schematic of movement of particles in different flow fields.

Figure 2.2. Potential energy curve for colloidal interaction.

Figure 2.3. Schematic of difference between rectilinear and curvilinear trajectories during orthokinetic agglomeration.

Figure 2.4. Collision efficiency as a function of shear rate and particle size.

Figure 4.1. Possible interactions between the surfaces of partially covered solid particles

Figure 4.2. Overlapping of adsorbed polymer layer as particles approach each other

Figure 5.1. Particle size distributions for original and sieved hollow spherical glass beads.

Figure 5.3. Reduced viscosity versus concentration of PEO to determine the intrinsic viscosity.

Figure 5.4. Adsorption isotherms for PEO on polystyrene. Absorbed amount ( $\Gamma)$ vs. equilibrium polymer concentration $\left(c_{\mathrm{p}}\right)$. x PEO 1500, o PEO 20,000, • PEO 300,000; (a) from water at $25^{\circ} \mathrm{C}$; (b) from $0.26 \mathrm{~mol} / 1 \mathrm{Mg} \mathrm{SO} 4$ solution at $25^{\circ} \mathrm{C}$ and $40^{\circ} \mathrm{C}$ (from Ref. [48]).

Figure 5.5 Turbidimetric calibration curve for 400,000 MW in glycerin-water solution

Figure 6.1. Image of particles in a freshly prepared suspension. Solid content $=0.2$ $\% \mathrm{v} / \mathrm{v}$

Figure 6.2. Image of agglomerates formed after 4 minutes of shearing at $80 \mathrm{~s}^{-1}$.

Solid content $=0.2 \% \mathrm{v} / \mathrm{v}$, PEO conc. $=100 \mathrm{mg} / \mathrm{l}, \mathrm{MW}=1 \times 10^{6}$. 
Figure 6.3. Agglomerate growth with respect to time at (a) $20 \mathrm{~s}^{-1}$, (b) $80 \mathrm{~s}^{-1}$ and (c) $190 \mathrm{~s}^{-1}$. Solid content $=0.2 \% \mathrm{v} / \mathrm{v}$, PEO MW $=1 \times 10^{6}$, PEO concentration $=$ $100 \mathrm{mg} / \mathrm{l}$.

Figure 6.4. Relative count of particles versus time of shearing at shear rate $=80 \mathrm{~s}^{-1}$. PEO concentration $20 \mathrm{mg} / \mathrm{l}$, solid content $=0.2 \% \mathrm{v} / \mathrm{v}, \mathrm{MW}=1 \times 10^{6}$.

Figure 6.5. Effect of polymer concentration on the collision efficiency. The suspension contained $0.2 \% \mathrm{v} / \mathrm{v}$ solid particles and MW of PEO was $1 \times 10^{6}$.

Figure 6.6. Effect of shear rate on the collision efficiency at different PEO

concentrations. PEO MW $=1 \times 10^{6}$ and solid content $=0.2 \% \mathrm{v} / \mathrm{v}$.

Figure 6.7. Effect of MW of PEO on the collision efficiency ( PEO conc. $=100 \mathrm{mg} / \mathrm{l}$, solid content $=0.2 \% \mathrm{v} / \mathrm{v})$.

Figure 6.8. Images of agglomerates at equilibrium formed at (a) 20 (b) 80 and (c) $190 \mathrm{~s}^{-1}$. Solid content $=0.2 \% \mathrm{v} / \mathrm{v}$, PEO conc. $=100 \mathrm{mg} / 1, \mathrm{MW}=1 \times 10^{6}$.

Figure 6.9. Effect of shear rate on maximum agglomerate size. Solid content $=$ $0.2 \% \mathrm{v} / \mathrm{v}, \mathrm{PEO}$ conc. $=100 \mathrm{mg} / \mathrm{l}, \mathrm{MW}=1 \times 10^{6}$.

Figure 6.10. Agglomerate growth when using different molecular weight PEO.

Shear rate $=80 \mathrm{~s}^{-1}$, solid content $=0.2 \% \mathrm{v} / \mathrm{v}$, PEO concentration $=100 \mathrm{mg} / \mathrm{l}$.

Figure 6.11. Effect of MW of PEO on maximum agglomerate size.

Figure 6.12. Reduction in the size of agglomerates formed at $20 \mathrm{~s}^{-1}$ after step up in shear rate.

Figure 6.13. Reduction in the size of agglomerates formed at $80 \mathrm{~s}^{-1}$ after step up in shear rate.

Figure 6.14. Agglomerate size distribution for the agglomerates produced at $20 \mathrm{~s}^{-1}$ 
and then after breakage at $190 \mathrm{~s}^{-1}$. 


\title{
List of Symbols
}

\author{
Latin \\ A \\ $A^{*} \quad$ Numerical function of interparticle distance (-) \\ $\mathrm{b} \quad$ Radius of solid particle (m) \\ $\mathrm{B}^{*} \quad$ Numerical function of interparticle distance (-) \\ $\mathrm{B}_{\mathrm{ij}} \quad$ Breakage fraction (-) \\ c Concentration $\left(\mathrm{kg} / \mathrm{m}^{3}\right)$ \\ $\mathrm{C}_{\mathrm{A}} \quad$ Attractive dimensionless parameter(-) \\ $\mathrm{C}_{\mathrm{R}} \quad$ Repulsive dimensionless parameter(-) \\ $\mathrm{C}_{\mathrm{S}} \quad$ Steric dimensionless parameter(-) \\ $\mathrm{C}^{*} \quad$ Numerical function of interparticle distance (-) \\ D Diffusion coefficient $\left(\mathrm{m}^{2} / \mathrm{s}\right)$ \\ $\mathrm{d}_{\mathrm{a}} \quad$ Diameter of agglomerate $(\mathrm{m})$ \\ E Rate of deformation tensor $\left(\mathrm{s}^{-1}\right)$ \\ $\mathrm{E}_{\text {turbulent }} \quad$ Average energy dissipation in turbulent flow $\left(\mathrm{J} / \mathrm{m}^{3}\right)$ \\ $\mathrm{F} \quad$ Force $(\mathrm{N})$ \\ $\Delta \mathrm{G} \quad$ Gibb's free energy (J) \\ $\mathrm{h}_{1}, \mathrm{~h}_{2} \quad$ Functions of relative size of particles (-) \\ $\Delta \mathrm{H} \quad$ Enthalpy of mixing $(\mathrm{J})$ \\ $\mathrm{J} \quad$ Collision frequency $\left(\mathrm{s}^{-1}\right)$ \\ $\mathrm{k}_{\mathrm{B}} \quad$ Boltzmann's constant $(\mathrm{J} / \mathrm{K})$ \\ $\mathrm{K}_{\mathrm{A}} \quad$ Constant in Argaman-Kaufman equation $\left(\mathrm{s}^{-1}\right)$ \\ $\mathrm{K}_{\mathrm{B}} \quad$ Constant in Argaman-Kaufman equation (-) \\ $1_{1}, l_{2}, l_{3} \quad$ Exponents in equation (3.15) (-) \\ $\mathrm{M}_{\mathrm{w}} \quad$ Molecular weight of polymer ( $\left.\mathrm{gm} / \mathrm{mole}\right)$ \\ n Number of sites (-) \\ n $\quad$ Unit vector (-) \\ $\mathrm{n}_{1} \quad$ Number of molecules of solvent (-) \\ $\mathrm{n}_{2} \quad$ Number of molecules of polymer (-) \\ $\mathrm{N} \quad$ Number concentration of particles $\left(\mathrm{m}^{-3}\right)$ \\ $\mathrm{N}_{\mathrm{A}} \quad$ Avagadro's number $\left(\mathrm{mol}^{-1}\right)$ \\ $\mathrm{P}_{0} \quad$ Amount of polymer added $(\mathrm{kg})$ \\ $\mathrm{r} \quad$ Distance $(\mathrm{m})$ \\ $\mathrm{r}^{*} \quad$ Dimensionless distance (-) \\ $<\mathrm{r}>\quad$ Mean end-to-end distance of polymer molecule (m) \\ $\mathrm{R}_{\mathrm{g}} \quad$ Radius of gyration $(\mathrm{m})$ \\ $\mathrm{s} \quad$ Distance between the surfaces of particles (m) \\ S Cross-sectional area $\left(\mathrm{m}^{2}\right)$ \\ $\mathrm{S}_{\mathrm{i}} \quad$ Splitting frequency $\left(\mathrm{s}^{-1}\right)$ \\ $\Delta \mathrm{S} \quad$ Entropy $(\mathrm{J} / \mathrm{K})$ \\ $\mathrm{t} \quad$ Time (s) \\ $\mathrm{t}^{*} \quad$ Dimensionless time (-) \\ T Temperature (K) \\ $\mathrm{u} \quad$ Relative velocity of particles $(\mathrm{m} / \mathrm{s})$
}




$\begin{array}{ll}\mathrm{v} & \text { Velocity of particles }(\mathrm{m} / \mathrm{s}) \\ \mathrm{v}_{1} & \text { Volume fraction of solvent }(-) \\ \mathrm{v}_{2} & \text { Volume fraction of polymer }(-) \\ \mathrm{V} & \text { Potential energy }(\mathrm{J}) \\ \mathrm{V}_{1} & \text { Molecular volume of solvent }\left(\mathrm{m}^{3}\right) \\ \mathrm{W} & \text { Exponent in collision efficiency model (-) } \\ \mathrm{x}_{1}, \mathrm{x}_{2}, \mathrm{x}_{3} & \text { Exponents in collision efficiency model (-) } \\ \mathrm{y}_{1}, \mathrm{y}_{2}, \mathrm{y}_{3} & \text { Exponents in collision efficiency model (-) } \\ \mathrm{z} & \text { Axial distance }(\mathrm{m}) \\ \mathrm{z} & \text { Co-ordination number }(-) \\ \mathrm{Z}_{\mathrm{P}} & \text { Fitting parameter in Deason's model (-) }\end{array}$

\section{Greek}

$\begin{array}{ll}\alpha & \text { Collision Efficiency }(-) \\ \beta & \text { Collision frequency }\left(\mathrm{s}^{-1}\right) \\ \dot{\gamma} & \text { Shear rate }\left(\mathrm{s}^{-1}\right) \\ \dot{\gamma}_{\text {extensional }} & \text { Strain rate }\left(\mathrm{s}^{-1}\right) \\ \Gamma & \text { Amount of polymer adsorbed }\left(\mathrm{gm} / \mathrm{m}^{2}\right) \\ \delta & \text { Thickness of polymer layer }(\mathrm{m}) \\ \Delta & \text { Constant in Berry's equation }\left([\mathrm{d} 1 / \mathrm{gm}]^{1.5} /[\mathrm{gm} / \mathrm{mol}]^{1.25}\right) \\ \varepsilon & \text { Porosity }(-) \\ \varepsilon_{\mathrm{r}} & \text { Relative dielectric permittivity }(-) \\ \varepsilon_{0} & \text { Dielectric permittivity of vacuum }\left(\mathrm{C}^{2} / \mathrm{Nm}^{2}\right) \\ \theta & \text { Fractional surface coverage }(-) \\ \theta^{*} & \text { Dimensionless spherical co-ordinate }(-) \\ \Theta & \text { Theta temperature }(\mathrm{K}) \\ \kappa & \left.\text { Debye-Huckel parameter (m }{ }^{-1}\right) \\ \Lambda & \text { Relative size of particles }(-) \\ \eta & \text { Shear Viscosity (kg/ms) } \\ \xi & \text { Expansion factor }(-) \\ \rho & \left.\text { Density (kg/m }{ }^{3}\right) \\ \sigma_{\mathrm{s}} & \text { Tensile strength }\left(\mathrm{N} / \mathrm{m}^{2}\right) \\ \psi_{0} & \text { Surface potential }(\text { Volt }) \\ \phi & \text { Volume fraction }(-) \\ \phi_{\mathrm{T}} & \text { Total volume of the particles }\left(\mathrm{m}^{3}\right) \\ \phi^{*} & \text { Dimensionless spherical co-ordinate }(-) \\ \varphi & \text { Fraction of active sites }(-) \\ \chi & \text { Flory-Huggin's parameter (-) }\end{array}$




\section{Chapter 1. Introduction}

A variety of chemical processes involve and many products consist of fine solid particles dispersed in a liquid medium in the form of a suspension. During processing, handling and transportation, a suspension often undergoes shear flow, which causes the dispersed particles to collide with each other. If a binding mechanism is present between the particles, they stick to each other to form agglomerates or flocs. This shear induced agglomeration of particles is also known as orthokinetic agglomeration. The stability of a suspension with respect to agglomeration during shear flow is an industrially significant phenomenon of particulate science, because whether particles remain well-dispersed as individual entities or agglomerate to form large aggregates, greatly influences the properties of the final product. For example, dispersions of solid particles of pigments and fillers in paint formulations or polymer solutions undergo shear flow during handling (coating), processing (injection molding) and transportation (flow in pipelines). To ensure a uniform quality product, it is necessary that a suspension be stable and not agglomerate. On the other hand, in solid-liquid separation operations such as waste-water treatment, a suspension is intentionally destabilized and subjected to shear flow through mechanical agitation so that small-size solid particles can agglomerate to form large flocs which are easily separated.

The nature and magnitude of various forces acting on the particles determine the stability of the suspension. Generally, in case of a solid dispersion in an aqueous medium, the forces acting on a particle include van der Waals forces, electrostatic forces and hydrodynamic forces. Of these, the van der Waals forces are attractive in nature and favor agglomeration. Electrostatic forces exist due to the presence of an electric double-layer 
around the particles and cause repulsion between the particles, and this has the effect of opposing agglomeration. Both these forces are similar in magnitude and act over comparable distances from the particle surface. If the electric double-layer repulsion dominates, the suspension remains stable and does not agglomerate. However, a suspension can be destabilized by suppressing the electric double-layer by adding an electrolyte such as $\mathrm{NaCl}$. This makes the van der Waals attractive forces dominant and the suspension undergoes agglomeration. This kind of agglomeration is known as "coagulation".

However, if a polymer is present in the suspension, additional effects can influence the stability of the suspension. If long chain polymer molecules dissolved in the suspending medium preferentially adsorb onto the solid surface of the particles, their loops and tails protrude into the liquid medium and depending on the nature of interaction between the polymer molecules, resulting effects may range from bridging flocculation to steric stabilization. When two particles approach each other, the polymer may form a bridge between them, holding them together. This mechanism of agglomerate formation is known as "polymer bridging" and this kind of agglomeration is also referred to as "flocculation". The efficiency of flocculation depends on the extent of coverage of the particle surface by the polymer and the quality of polymer-solvent interaction. Quite often suspensions encountered in practice are aqueous in nature where water is a good solvent for the dissolved polymer. In this case, the flocculation process is more effective when surfaces of the colliding particles are only partially covered with the polymer. When the particle surface is totally covered with the polymer, colliding particles do not stick as the polymer molecules repel each other and the suspension remains stable. This is 
known as steric stabilization. If the suspension in question is such that the dispersing medium is a not a good solvent for the adsorbed polymer, polymer layers attract each other and flocculation is possible.

In some cases added polymer does not adsorb on the particles, but flocculation can still take place. When two particles approach each other at distances shorter than the size of the polymer molecule, the polymer is excluded from the region between the particles. This results in a net attraction due to the lower osmotic pressure in the interstitial region, and this leads to "depletion flocculation". The present research is only concerned with coagulation and bridging flocculation phenomena, and depletion flocculation will not be considered.

Suspensions containing polymers are widely encountered in many industrial products and processes where flocculation and steric stabilization are found to be important. For example, polymers are used as stabilizers in paints, pharmaceuticals and cosmetic creams where agglomeration of dispersed solid particles must be prevented. On the other hand, flocculation by polymers is exploited where size-enlargement is required such as in water purification, mineral processing and paper manufacturing [1].

Here it must be recognized that when particles collide with each other, not all collisions taking place successfully result in the formation of agglomerates; rather, only a fraction of the total collisions lead to the formation of agglomerates, and this fraction is known as the collision efficiency and it determines the overall rate of agglomeration.

When a suspension is sheared, the particulate size increases as agglomeration takes place. However, stresses developed at the same time due to fluid shearing tend to break up the agglomerates to a smaller size. Hence, during the flow of a suspension, 
particle enlargement and breakage take place simultaneously. Consequently, agglomerates do not continue to grow indefinitely in size during prolonged shearing; instead they attain an equilibrium size.

Clearly, two parameters of importance in designing an agglomeration process arethe collision efficiency and the agglomerate equilibrium size. Both of these parameters depend on the physicochemical and hydrodynamic characteristics of the system, and these include the particle size (colloidal or non-colloidal), mechanism of agglomeration (coagulation or polymer bridging) and type of flow (laminar or turbulent).

This project was motivated by the need to understand the process of agglomeration of pigments and fillers dispersed in a polymer solution when the suspension is subject to shear flow during transportation through a pipeline. In this case, one is dealing with a suspension of non-colloidal particles subject to laminar shear flow where the agglomeration takes place due to polymer bridging. In this dissertation, a literature review is presented that describes the current understanding of the polymer bridging process in terms of collision efficiency and maximum agglomerate size. This is followed by the development of a new theoretical model for collision efficiency. Experimental results are then presented using a model suspension system to validate the model and to show the effects of various parameters on the collision efficiency and the floc size. it is emphasized that apart from agglomeration of particles in a polymer solution, the results obtained in this work can also applied to other particulate systems where adsorbed polymer affects agglomeration behavior. 


\section{Chapter 2. Background of Agglomeration Processes}

For agglomeration of particles to take place, two processes must occur- at first, particles must come in close proximity of each other or "collide" with each other, and second, under the influence of colloidal forces they must stick to each other to form an agglomerate. Therefore, the general form of rate of agglomeration is given as:

$-\frac{d N}{d t}=\{$ collision efficiency $\}$ $\{$ collision frequency $\}=\alpha \times \mathrm{J}$

In equation (2.1) $\mathrm{N}$ is the number concentration of particles or agglomerates at time $\mathrm{t}$. Also, $\mathrm{J}$ is the collision frequency and it represents the number of collisions taking place per unit time; this depends on the mode by which particle displacement with respect to each other is taking place, which largely depends on the physical characteristics of the system. Finally, the collision efficiency $\alpha$, reflects the fraction of total number of collisions that successfully result in the formation of agglomerates and it depends on the relative magnitudes of physical and colloidal forces. In the following sections both factors determining the rate of agglomeration are discussed in detail.

\subsection{Perikinetic Collision Frequency}

If in a suspension, particle-particle collisions take place solely due to Brownian motion of the particles, the phenomenon is known as perikinetic coagulation. Brownian motion occurs due to thermal energy and it becomes increasingly important when particles are very small- of the order of a micron or smaller. Smoluchowski derived an expression for collision frequency in this case by considering the diffusive flux of the particles towards a stationary particle. Using Fick's first law for the number of particles J' going through a unit area toward a reference particle per unit time [2]: 


$$
J^{\prime}=-\mathrm{D} \frac{d N}{d r}
$$

where $\mathrm{D}$ is the diffusion coefficient of particles, $\mathrm{N}$ is number concentration and $\mathrm{r}$ is the radial coordinate. The number of particles going through a sphere of radius $r$ in unit time is:

$$
J^{\prime \prime}=-\left(4 \pi r^{2}\right) \mathrm{D} \frac{d N}{d r}
$$

The left hand side of the above equation is constant under steady state conditions. Therefore equation 2.3 can be integrated easily to relate $\mathrm{N}$ to $\mathrm{r}$ under the boundary conditions that $\mathrm{N}=\mathrm{N}_{0}$ at $\mathrm{r}=\infty$ and $\mathrm{N}=0$ at $\mathrm{r}=2 \mathrm{~b}$ where $\mathrm{b}$ is the radius of particles. Clearly, any particle whose center lies within the distance $2 b$ of the target particle is captured. Integration of equation (2.3) leads to:

$$
J^{\prime \prime}=-8 \pi b \mathrm{D} N_{0}
$$

So far it has been assumed that the target particle was stationary, but it also undergoes diffusion resulting in a relative diffusion coefficient of $2 \mathrm{D}$. Now for all $\mathrm{N}$ particles present, the rate of agglomeration is given by:

$$
J=-8 \pi b \mathrm{D} N_{0}^{2}
$$

where a factor of $1 / 2$ was included in above expression to account for the fact that each collision is counted twice when calculating the total flux. Now the diffusion coefficient of the particles is given by the well-known Stokes-Einstein expression [2]:

$\mathrm{D}=\frac{k_{B} T}{6 \pi \eta b}$

where, $\mathrm{k}_{\mathrm{B}}$ is Boltzmann's constant, $\mathrm{T}$ is temperature and $\eta$ is the viscosity of the dispersing medium. Introducing equation (2.6) in equation (2.5) gives the expression for 
perikinetic collision frequency $\mathrm{J}_{\mathrm{Br}}[3]$, where subscript $\mathrm{Br}$ represents the movement of particles due to Brownian motion:

$$
J_{B r}=-\frac{4 k_{b} T}{3 \eta} N_{0}{ }^{2}
$$

The above equation shows that increasing the temperature causes the perikinetic collision frequency to increase whereas increasing the viscosity of the medium reduces the collision frequency.

Since the collision frequency is second order in terms of number concentration, it falls off rapidly with $\mathrm{N}$ as particles agglomerate to form doublets and triplets. Also as the agglomerate size increases, their diffusivity, as given by the Stokes-Einstein equation, decreases. These factors limit the size to which agglomerates grow by the process of perikinetic agglomeration.

\subsection{Differential Sedimentation Collision Frequency}

If the densities of the particles and the suspending medium are dissimilar, particles may float or settle. Particles of different diameters settle at different velocities causing the faster moving particles to collide with slower moving particles leading to agglomeration. By balancing the forces of gravity, buoyancy and drag, the sedimentation velocity of a particle of radius $b_{i}$ and density $\rho_{i}$ in a medium of density $\rho$ is given by Stokes' equation [4]:

$v_{i}=\frac{2 g}{9} \frac{\left(\rho_{i}-\rho\right) b_{i}^{2}}{\eta}$

The relative velocity between two particles of diameters $b_{i}$ and $b_{j}$ would be $u=v_{i}-v_{j}$. The rate of $N_{i}$ particles through a cylindrical cross section of $\left(b_{i}+b_{j}\right)$ is given by [5]: 
$\frac{d N_{i}}{d t}=N_{i} \pi\left(b_{i}+b_{j}\right)^{2}\left(v_{i}-v_{j}\right)$

Using equations (2.8) and (2.9) the sedimentation collision frequency $\mathrm{J}_{\text {sedimentation }}$ is expressed as [5]:

$J_{\text {se dimentation }}=-\frac{2 \pi g}{9 \eta}\left(b_{i}+b_{j}\right)^{3}\left|b_{i}-b_{j}\right|\left(\rho_{s}-\rho\right) N_{i} N_{j}$

where $\mathrm{N}_{\mathrm{i}}$ and $\mathrm{N}_{\mathrm{j}}$ are the number concentrations of particles of radii $b_{i}$ and $b_{j}$ respectively. It must be pointed out that the differential sedimentation is significant in solid-liquid separation processes such as mineral or ore suspension where solid particles have a significantly larger density than the aqueous medium.

\subsection{Orthokinetic Collision Frequency}

If the particle motion takes place solely due to the presence of a velocity gradient within the fluid, it is known as orthokinetic flocculation. In this case the collision frequency depends on the nature of the flow. In a steady laminar shear flow, a constant velocity gradient exists and the particles flowing along the different streamlines move at different velocities and collide with each other as shown schematically in Figure (2.1a). Here, it is assumed that the path of each particle remains rectilinear and streamlines are not disturbed by the presence of particles. Smoluchowski was the first to calculate the collision frequency in shear flow. If the shear rate is $\dot{\gamma}$, then any particle at a distance $\mathrm{z}$ away from the center of the target particle would have the velocity $\dot{\gamma} \mathrm{z}$. The particle flow through the segment dz as shown in Figure (2.1a) is [5]:

$d\left(\frac{d N}{d t}\right)=2 \dot{L} N\left(4 b^{2}-z^{2}\right)^{1 / 2} z d z$ 


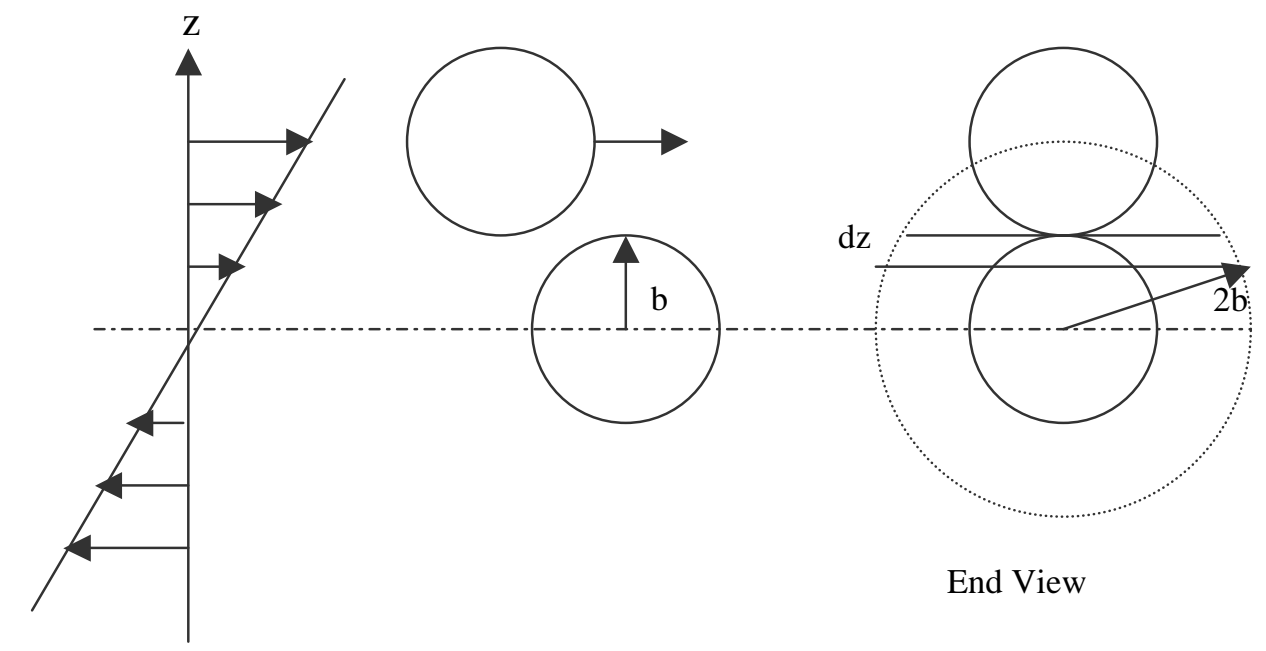

(a) Movement of particles in shear flow

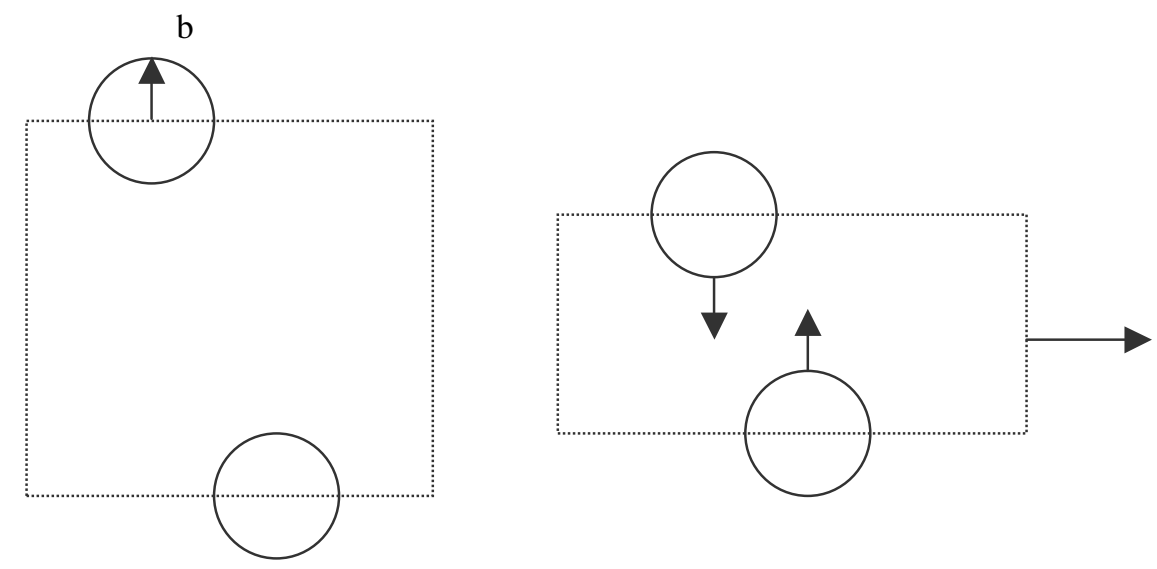

(b) Movement of particles in extensional flow

Figure 2.1. Schematic of the movement of particles in different flow fields. 
Considering that any particle whose center lies within the distance $2 \mathrm{~b}$ from the center of the target particle will be captured and that flow is taking place in both the upper and lower hemispheres, the above equation can be integrated from $\mathrm{z}=0$ to $\mathrm{z}=2 \mathrm{~b}$ to obtain [5]:

$$
\begin{gathered}
\frac{d N}{d t}=-4 \dot{\mu N} \int_{0}^{2 b} z\left(4 b^{2}-z^{2}\right)^{1 / 2} d z \\
=-\frac{32}{3} \dot{N} b^{3}
\end{gathered}
$$

This is the collision frequency experienced by one particle. Since there are N such particles in the suspension, the net orthokinetic collision frequency $\mathrm{J}_{\text {shear }}$ is given by:

$$
J_{\text {Shear }}=-\frac{16}{3} N^{2} \dot{b b}^{3}
$$

The other major flow field of interest is extensional flow and Figure (2.1b) shows the movement of the particles in pure elongational flow which results in the collisions between the particles. In this case the collision frequency is given by [6]:

$$
J_{\text {Shear }}=-\frac{16 \pi}{3} \dot{\gamma}_{e x t} b^{3} N^{2}
$$

where, $\dot{\gamma}_{e x t}$ is the strain-rate.

Usually, it is assumed that the three mechanisms of interparticle collisions are independent and when they operate simultaneously the agglomeration rates are additive [7]:

$$
J_{\text {total }}=J_{B r}+J_{\text {sedimentation }}+J_{\text {Shear }}
$$


The relative magnitudes of each contribution depend on the characteristics of the suspension and the flow conditions. If the densities of the particles and the dispersing medium are nearly the same, contribution due to sedimentation can be neglected. Other factors that can limit the effect of sedimentation are high viscosity of the dispersing medium and the relatively small size of the particles.

Next, one must compare the collision frequencies due to shear flow that with due to Brownian motion. The ratio of their magnitude is characterized by Peclet number which is obtained by dividing equation (2.13) by (2.7) [8]:

$$
P e=\frac{4 \eta b^{3}}{k_{B} T}
$$

If $\mathrm{Pe}>>1$, shear flow dominates the flocculation, whereas for $\mathrm{Pe}<<1$, Brownian motion will dominate.

\subsection{Perikinetic Collision Efficiency}

Brownian motion of colloidal particles causes them to collide with each other. If no resistance to collisions is present, every collision leads to agglomeration and this is known as rapid coagulation. In this case, collision efficiency $\alpha=1$, and the suspension is termed completely unstable. The stability of dispersions of fine particles in a liquid medium depends on the short-range interparticle forces. The net force is the sum of van der Waals attractive forces and electric double-layer repulsive forces. Total interaction between them can be described in terms of a potential energy function. The van der Waals attraction potential energy between two spheres of equal size is given by [9]:

$$
V_{A}=-\frac{A}{6}\left[\frac{2 b^{2}}{r^{2}-4 b^{2}}+\frac{2 b^{2}}{r^{2}}+\ln \left(\frac{r^{2}-4 b^{2}}{r^{2}}\right)\right]
$$


where $r$ is the center to center distance between the particles, $b$ is the radius of each particle and A is the Hamaker constant. The negative sign indicates that the potential is attractive in nature. The potential energy due to the electric double-layer can be expressed as [10]:

$$
V_{R}=2 \pi \varepsilon b \psi_{0}^{2} \ln \left\{1+\exp \left[-\kappa b\left(\frac{r}{b}-2\right)\right]\right\}
$$

where $\varepsilon$ is the dielectric constant, $\psi_{0}$ is surface potential and $\kappa$ is the Debye-Huckel parameter. Clearly, the total potential energy of interaction $V_{T}=V_{A}+V_{R}$ is a function of separation distance between the particles. A schematic diagram of general shape of the total potential energy with respect to the separation distance is shown in Figure (2.2). If a particle can overcome the energy barrier it coagulates in the primary minimum. In this case attraction is very strong and redispersion of the particles is almost impossible. However, coagulation in the secondary minimum is also possible and it results in the formation of weak aggregates. If repulsive forces are large enough to reduce the secondary minimum, the suspension can remain well dispersed. Note that coagulation can be induced by adding an electrolyte (also known as the coagulant) which suppresses the electric double layer repulsion, thus reducing the energy barrier and destabilizing the suspension.

The potential energy barrier slows down the flux of particles and appears as a resistance term in equation (2.3):

$$
J^{\prime \prime}=-\left(4 \pi r^{2}\right) \mathrm{D} \frac{d N}{d r}+\text { resistance term }
$$



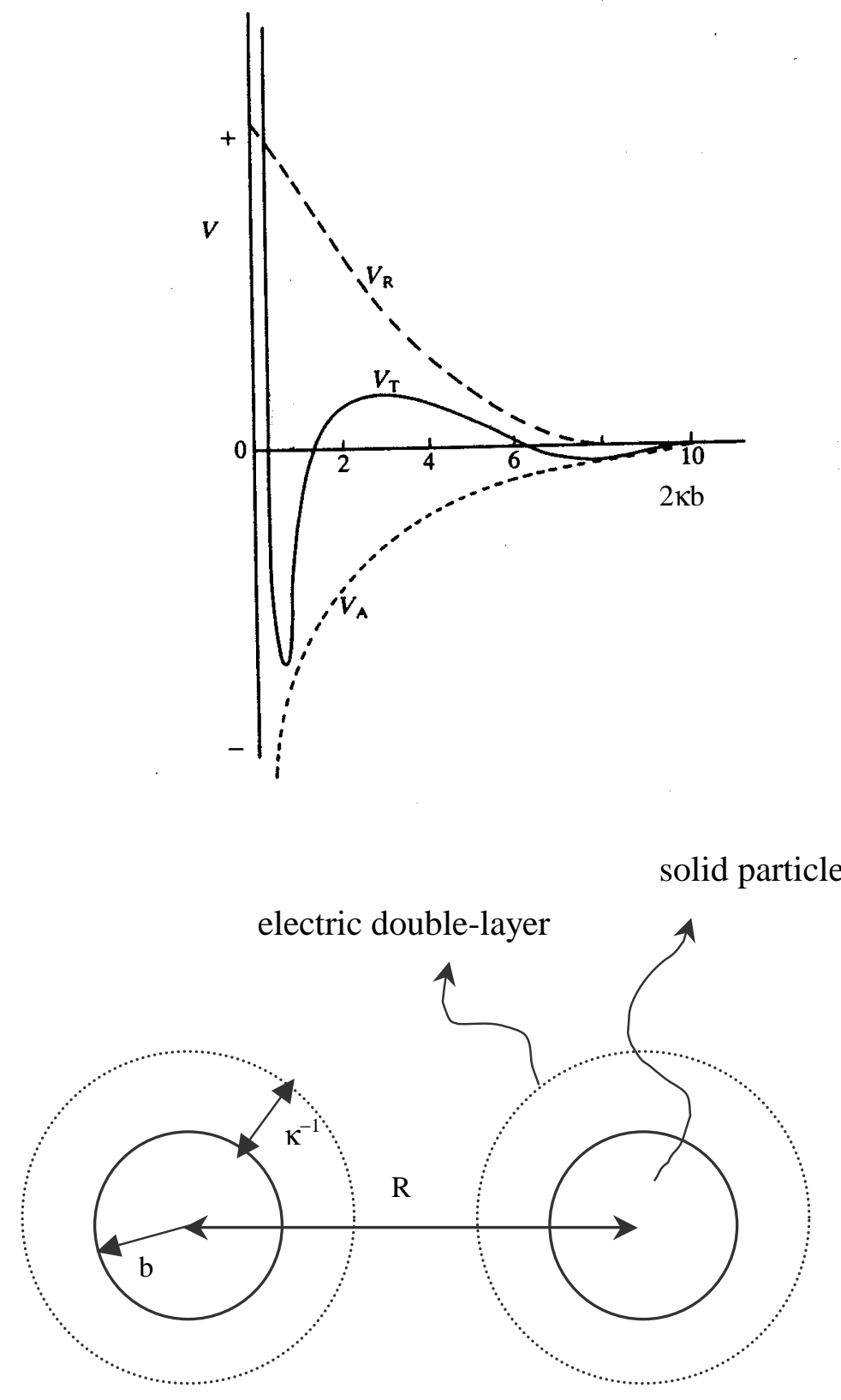

Figure 2.2. Potential energy curve for colloidal interaction. 
The force of resistance is obtained by differentiating the potential energy function with respect to interparticle distance. The force of resistance divided by the friction factor gives the velocity of particles, which multiplied by the area of spherical shell of radius $r$ yields [2]:

$J^{\prime \prime}=-4 \pi r^{2}\left(\mathrm{D} \frac{d N}{d r}+\frac{N D}{k_{B} T} \frac{d V_{T}}{d r}\right)$

The above differential equation can be solved under appropriate boundary conditions to obtain the rate of agglomeration as [2]:

$$
J_{B r}^{\prime}=\frac{-8 \pi D N^{2}}{\int_{2 b}^{\infty} \exp \left(\frac{V_{T}}{k_{B} T}\right) \frac{d r}{r^{2}}}
$$

Comparing equation (2.7) with equation (2.21) and using equation (2.6) for the expression of $\mathrm{D}$, the collision efficiency, $\alpha$, can be expressed as [5]:

$$
\frac{1}{\alpha}=2 \int_{2}^{\infty} \exp \left(\frac{V_{T}}{k_{B} T}\right) \frac{d(R / b)}{(R / b)^{2}}
$$

In some of the literature on colloid science (see for example [2], [8]), the term (1/ $\alpha$ ) is referred to as the stability ratio and it ranges from unity for a completely destabilized suspension to infinity for a completely stable suspension. 


\subsection{Orthokinetic Collision Efficiency}

Smoluchowski's treatment assumes that particles travel in straight trajectories along streamlines, i.e., streamlines are not disturbed by the presence of particles. However, due to van der Waals, electric double-layer and hydrodynamic interactions, particle trajectories deviate from a straight line as particles approach each other. Schematically, the difference between the rectilinear and curvilinear trajectories is shown in Figure (2.3).

If trajectories are straight, any particle whose center lies within a distance $2 b$ from the center of the target particle is captured and the trajectory is called closed. Any particle farther than $2 b$ is not captured, and the trajectory is considered open. Thus, the capture cross-section is of the order of $\pi \mathrm{b}^{2}$. If trajectories become curved in nature due to various forces, not all particles lying within $2 \mathrm{~b}$ are captured, and the capture cross-section can be much less than $\pi \mathrm{b}^{2}$. This capture cross-section is determined by a trial and error method by calculating the trajectories which are closed. By comparing the capture cross-section in the curvilinear case with that in the rectilinear case, the collision efficiency can be determined for doublet formation.

Batchelor and Green [11] theoretically considered only the effect of hydrodynamics on the trajectories of the particles in both shear and elongational flow. It was found that hydrodynamic forces retard the encounter between the particles because the liquid layer between the particles has to flow out and this imposes a viscous resistance causing the streamlines to become curved near the particle surface. 


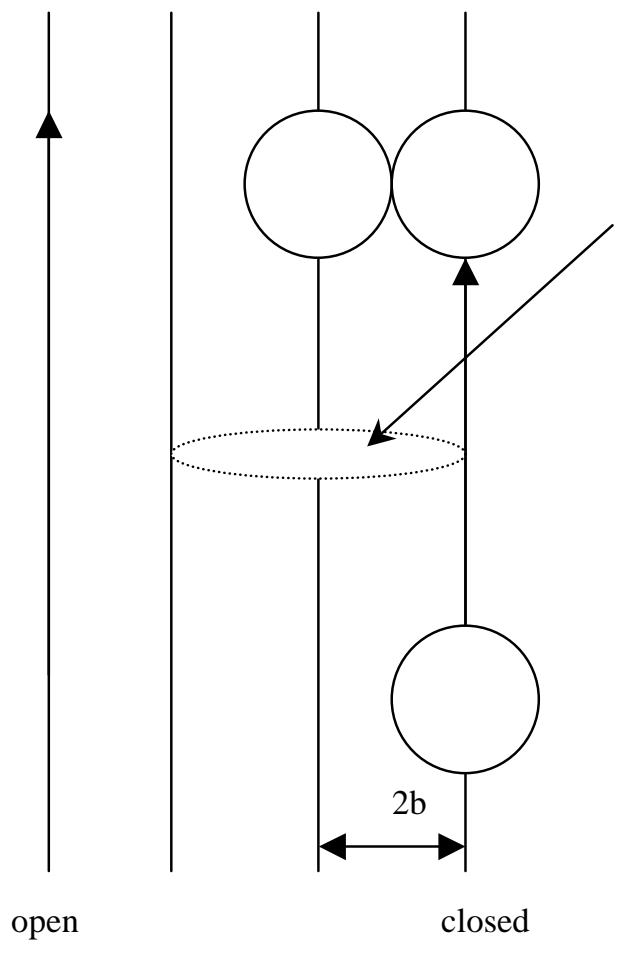

Rectilinear Trajectories



Curvilinear Trajectories

Figure 2.3. Schematic of the difference between rectilinear and curvilinear trajectories during orthokinetic agglomeration. 
Van de Ven and Mason [12] and Zeichner and Schowalter [8] incorporated the effects of van der Waals attraction and electric double-layer repulsion forces on the trajectories of the particles. The net velocity of a particle is given as the sum of velocity fields which are independent and superposable [8]:

$\mathrm{u}=\mathrm{u}_{\mathrm{flow}}+\mathrm{u}_{\mathrm{c}}$

The velocity field $\mathrm{u}_{\text {flow }}$ is due to the hydrodynamic flow while $\mathrm{u}_{\mathrm{c}}$ is due to the presence of colloidal forces. In a spherical coordinate system, the trajectory of a particle approaching a target particle is given by [12]:

$$
\begin{aligned}
& \frac{d r^{*}}{d t^{*}}=A^{*}\left(r^{*}\right) \sin ^{2} \theta^{*} \sin 2 \phi^{*}+C^{*}\left(r^{*}\right)\left[\frac{F_{\text {int }}(r)}{3 \pi \eta \dot{\gamma b}^{2}}\right] \\
& \frac{d \theta^{*}}{d t^{*}}=\frac{B^{*}\left(r^{*}\right)}{4} \sin 2 \theta^{*} \sin 2 \phi^{*} \\
& \frac{d \phi^{*}}{d t^{*}}=\frac{1}{2}\left\{1+B^{*}\left(r^{*}\right) \cos 2 \phi^{*}\right\}
\end{aligned}
$$

here, $\mathrm{A}^{*}\left(\mathrm{r}^{*}\right), \mathrm{B}^{*}\left(\mathrm{r}^{*}\right)$ and $\mathrm{C}\left(\mathrm{r}^{*}\right)$ are the monotonically increasing functions of distance between the centers of the particles $\left(\mathrm{r}^{*}\right)$. $\mathrm{F}_{\mathrm{int}}(\mathrm{r})$ is the total interaction force between the particles which is obtained by differentiating the total interaction energy between the particles:

$$
F_{\text {int }}=-\frac{\partial V_{\text {int }}}{\partial r}
$$

The total interaction energy is the sum of the van der Waals attraction and electric double-layer repulsion as given by equations (2.17) and (2.18). If one differentiates these equations with respect to interparticle distance and makes the result dimensionless (as shown in a later section), two dimensionless parameters are obtained. Repulsion parameter 
$C_{R}=\frac{2 \varepsilon_{r} \varepsilon_{0} \psi_{0}^{2}}{3 \eta \dot{\gamma b}}$

\section{Attraction parameter}

$$
C_{A}=\frac{A}{36 \pi \eta \dot{\gamma} b^{3}}
$$

$C_{R}$ and $C_{A}$ represent the relative importance of repulsion and attraction forces. A high value of $C_{R}$ inhibits coagulation whereas a high value of $C_{A}$ promotes coagulation. To obtain the collision efficiency, the differential equation given in equation (2.24) is solved as one particle moves from infinity towards the target particle. By trial and error, a crosssection area is found for which any particle passing through it ends up adhering to the target particle. This cross-section divided by the flow cross-section in the case of straight trajectory gives the collision efficiency. If the electric double-layer repulsion is completely suppressed by adding an electrolyte, numerical calculations give an easy expression for the collision efficiency [13]:

$\alpha=\mathrm{K}\left(\frac{A}{36 \pi \eta \dot{j^{3}}}\right)^{0.18}$

where $\mathrm{K}$ is a constant whose value is close to unity. This equation applicable only when $C_{R}=0$ and $10^{-5}<C_{A}<10^{-1}$. This equation has been found to be in good agreement with the experimental results $[13,14]$.

Besides shear flow, trajectory analysis has been carried out for uniaxial extension flow also [8]. It was found that the extensional flow is more efficient in causing flocculation as compared to shear flow. The reduction in collision efficiency in shear flow was attributed to the vorticity of the shear flow which reduces the time during which hydrodynamic forces and colloidal forces interact with each other. Greene et al. [15] 
incorporated a general function in trajectory analysis which accounts for all hydrodynamic flows ranging from purely rotational to extensional flow and showed its effect on the collision efficiency.

It should be noted that trajectory analysis is valid for calculating the collision efficiency of doublet formation resulting from two colliding primary particles. So far, no theoretical treatment is available to calculate the collision efficiency if agglomerates grow further from doublets to triplets and larger agglomerates because of the complexity of the hydrodynamics involved. Adler [16] used trajectory analysis to numerically calculate the collision efficiency when colliding particles are unequal in size. Coagulation between unequal size particles is known as heterocoagulation as opposed to homocoagulation between two equal size particles. The main conclusion of this study was that in most cases, homocoagulation is favored over heterocoagulation and larger the size difference between the colliding particles, the less likely they are to form an agglomerate. De Boer et al. [17] used Adler's [16] method to calculate the efficiencies among spheres of 1 and 2, 4 or $8 \mu \mathrm{m}$ diameter particles. The collision efficiency as a function of shear rate is shown in Figure (2.4), which shows that the likelihood of agglomerate formation between two particles that are significantly different in size is much less and falls of rapidly as the shear rate is increased. The implication of this is that during agglomeration, agglomerate growth at a later stage is most likely to take place due to collisions between agglomerates rather than between an agglomerate and a single particle. Brakalov [18] applied trajectory equations to the collisions between agglomerates assuming them to be spherical and impenetrable. It was suggested that the 


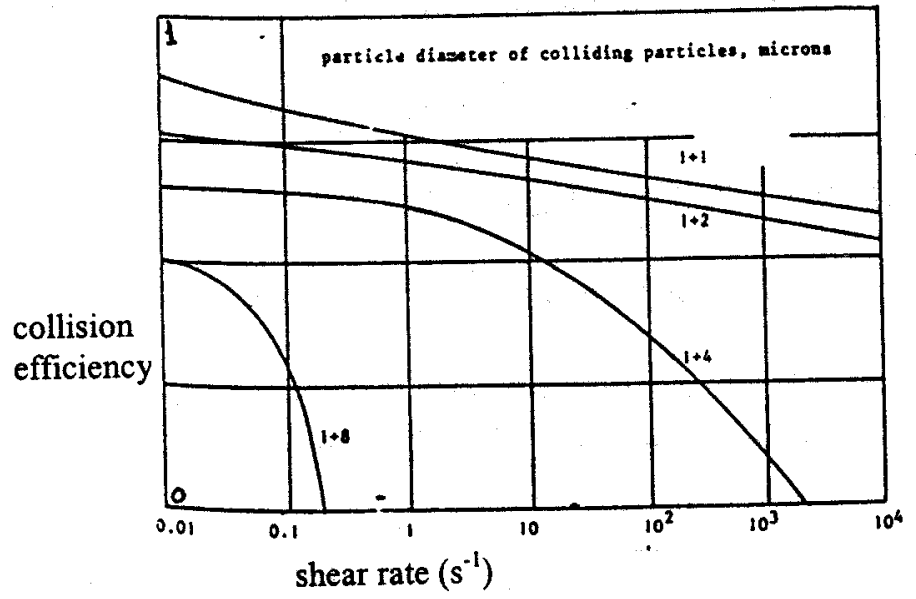

Figure 2.4 . Collision efficiency as a function of shear rate and particle size (from Ref. [17]). 
colloidal forces between the agglomerates are determined by a couple of primary particles whereas hydrodynamic forces are equivalent to forces one would expect between two particles of the size of complete agglomerates. Thus as the agglomerate size increases, hydrodynamic forces increase much more rapidly than colloidal forces resulting in a much lower agglomerate collision efficiency than primary particle collision efficiency. Potanin [19] suggested the following expression for the agglomeration collision efficiency:

$$
\alpha_{a} \approx \frac{2.1}{\left(\ln \left(d_{a} / 2 b\right)\right)^{0.29}}\left(\left(d_{a} / 2 b\right)^{0.075}-0.2\right)^{\frac{3}{2}}
$$

where $d_{a}$ is the diameter of the agglomerate. This expression shows that collision efficiency decreases with increasing $d_{a}$ with respect to primary particle size $2 b$.

\subsection{Orthokinetic Rate of Agglomeration}

From equations (2.1) and (2.13), the orthokinetic rate of agglomeration becomes:

$\frac{d N}{d t}=-\frac{16}{3} \alpha N^{2} \dot{\gamma b}$

If the volume fraction of particles $\phi$ is assumed to remain constant then at any instant, the number concentration of particles can be related to the particle size by $\phi=$ $(4 / 3) \pi \mathrm{b}^{3} \mathrm{~N}$. Now equation (2.27) can be integrated to obtain:

$$
\ln \frac{N}{N_{0}}=-\frac{4 \alpha \dot{\gamma} \phi}{\pi} t
$$

where $\mathrm{N}_{0}$ is the number concentration at time $\mathrm{t}=0$ and $\mathrm{N}$ is the number concentration at any time t. This equation forms the basis for experimentally determining the collision efficiency. By following the number concentration with respect to time, equation (2.28) 
gives a straight-line during the early stage of agglomeration. From the slope of this line experimental value of the collision efficiency, $\alpha$, can be determined.

From the information presented in the preceding sections, it can be seen that for the case of orthokinetic agglomeration caused by coagulation, detailed calculations can be made to determine the rate of agglomeration. However, the present work is concerned with orthokinetic agglomeration where particles bind together due to polymer bridging. In the following chapter a literature review is presented which lays out the current understanding of polymer bridging flocculation. It also shows how the methods to determine collision efficiency in the case of polymer bridging differ from coagulation. 


\section{Chapter 3. Review of Polymer Bridging Flocculation}

\subsection{Polymer Bridging}

The presence of long chain polymer molecules in a suspension has a profound effect on the stability of the dispersion. This is because adsorption of polymer molecules at the solid-liquid interface determines stability. Indeed, in general, fractional surface coverage by the polymer promotes flocculation whereas complete surface coverage stabilizes the dispersion. When a long chain polymer adsorbs on the surface, it assumes a conformation in the form of loops and tails which extend into the liquid medium. As the particles collide with each other during shear-flow, polymer segments on the surface of one particle become attached to the bare surface of the other particle, thus forming a bridge which holds them together.

When a polymer solution is added to a suspension that is being sheared, several processes may take place [20]:

1. Mixing of polymer molecules in the liquid phase surrounding the solid particles.

2. Transport of polymer to the solid-liquid interface followed by adsorption of the polymer on the solid surface.

3. Rearrangement of adsorbed chains on the solid surface.

4. Collision between the coated particles which may result in the formation of agglomerates.

5. Break up of flocs.

These processes do not occur in a sequential manner; rather they occur simultaneously, which makes the analysis of the overall process difficult. However in order to simplify the analysis, it is assumed that steps 1-2 occur quite rapidly with respect 
to the time scale of agglomeration. In fact, in the case of orthokinetic agglomeration at shear rates as low as $50 \mathrm{~s}^{-1}$, steps 1 and 2 may occur within a few seconds of adding the polymer flocculant and stirring [21]. Thus, it is reasonable to assume that the surface coverage of the solid particles by the flocculating polymer does not change with time during the agglomeration process.

Unlike the rigorous deterministic methods of efficiency calculation for the electrolytic coagulation process, collision efficiency calculations in polymer bridging have been based on probabilistic considerations derived from the fractional surface coverage of the particles by the polymer. In this methodology, it is proposed that bridging between two colliding particles can occur only when the covered surface of one particle comes in contact with the bare surface of another particle. If $\theta$ is the fraction of the solid surface that is covered by the adsorbed polymer then $(1-\theta)$ is the fraction of uncovered surface. In the simplest formulation, Smellie and La Mer [22] proposed that the collision efficiency is given by:

$$
\alpha=\theta(1-\theta)
$$

which is the probability of a covered surface coming in contact with a bare surface. This equation adequately describes the general features of the flocculation process; for example, it shows that both at zero coverage $(\theta=0)$ and at full coverage $(\theta=1)$, collision efficiency should be zero. However, this equation also predicts that the maximum efficiency occurs at $\theta=1 / 2$ and has a value of $1 / 4$, which is contrary to experimental evidence that shows that flocculation is more or less optimum over a range of surface coverage and can have values more than 0.25 [23]. Hogg [23] suggested that a factor of 2 should be introduced into the equation because if a covered area adheres to the bare 
surface of another particle then the reverse is also true. Therefore, the efficiency should be given by $2 \theta(1-\theta)$. To further improve this model, the concept of active sites was introduced which meant that polymer adsorption takes place only at few sites on the particle and that the interacting particles have complete freedom to reorient themselves into a configuration favorable for adhesion. Now the collision efficiency becomes [23]:

$$
\alpha=1-\theta^{2 \mathrm{n}}-(1-\theta)^{2 \mathrm{n}}
$$

where, $\mathrm{n}$ is the number of active sites on the particle surface which can be calculated by dividing particle surface area by the projected area of a polymer molecule:

$$
\mathrm{n}=4 \pi \mathrm{b}^{2} / \pi \mathrm{R}_{\mathrm{g}}{ }^{2}
$$

in which $\mathrm{R}_{\mathrm{g}}$ is the radius of gyration of polymer molecule. Usually a polymer molecule is much smaller than the solid particle. Therefore $\mathrm{n} \gg>1$, and it can be seen that $\alpha$ approaches unity over a range of $\theta$. Clearly, equation (3.2) overestimates the collision efficiency. It must be noted that for $\mathrm{n}=1$ this turns into the Smellie and La Mer model.

Moudgil et al. [24] introduced the concept of active and inactive sites into the formulation for collision efficiency. If it is assumed that only a fraction of total sites is active towards adsorption and interaction with polymer molecules, then the collision efficiency is given by:

$$
\alpha=2 \varphi^{2} \theta(1-\theta)
$$

where $\varphi$ is the fraction of active sites.

Several criticisms can be leveled against these methods of calculating collision efficiency ([25], [26]). First of all, they do not account for any hydrodynamic and electrostatic effects which are associated with the shear flow of suspensions. Furthermore, enhancement in the collision efficiency may occur due to increase in the 
effective radius of the particles resulting from the polymer loops and tails dangling from the surface. On the other hand, due to the electrostatic repulsion and hydrodynamic resistance, particles cannot come arbitrarily close to each other to form bridges. A few attempts have been made to incorporate these effects into probabilistic formulations of collision efficiency, and these are discussed below.

Trajectory analysis shows that the colliding particles do not come arbitrarily close to each other but that they approach a minimum distance $d_{\min }$ to each other. If $d_{\min }$ is such that the attractive forces dominate, flocculation takes place. For unequal size particles $d_{\min }$ is larger than for equal size particles. Van de Ven [27] suggested that increased flocculation efficiency in case of polymer bridging is due to enlargement in particle size because of the adsorbed polymer layer. The thickness of the polymer layer can be several nanometers in magnitude and if this is greater than $d_{\min }$, it would be able to cause flocculation.

Deason [28] suggested that the number of interacting sites between two approaching particles is determined by the size of the polymer molecule and $d_{\min }$. The number of interacting sites for two equal sized particles is obtained by calculating the area of polymer molecule accessible for bridging when particle surfaces are $d_{\min }$ apart. This is given by

$$
n=\frac{b\left(R_{g}-d_{\min }\right)}{R_{g}^{2}}
$$

where $\mathrm{R}_{\mathrm{g}}$ is polymer extension into the solution. Furthermore, only $\mathrm{m}$ sites out of $\mathrm{n}$ form the bridges and probability of that occurring is given by a binomial probability distribution: 


$$
P(m, n)=\frac{n !}{m !(n-m) !}[2 \theta(1-\theta)]^{m}[1-2 \theta(1-\theta)]^{n-m}
$$

The bridging will occur in all cases except $\mathrm{m}=0$, the bridging efficiency is given by:

$$
\alpha=1-P(0, n)=1-[1-2 \theta(1-\theta)]^{n}
$$

As can be seen this model takes into account the effect of polymer size and $d_{\text {min }}$ incorporates hydrodynamic effects. This model predicts collision efficiencies that are larger than that predicted by the Smellie and La Mer model but smaller than Hogg's model. But in the case of non-colloidal suspensions, solid particles are much larger than the polymer molecules and this makes $n>>1$ and the calculated collision efficiency approaches close to unity. This is usually the case with all the models discussed so far. They are more suited for colloidal suspensions where solid particles and the polymer molecules are comparable in size. For non-colloidal suspensions, a different model is needed.

Aunins [26] studied the flocculation of animal cells of average diameter of $7.8 \mu \mathrm{m}$ with the help of a cationic polyelectrolyte poly-L-histidine in the laminar flow regime. To calculate the collision efficiency the following model was used:

$$
\left.\alpha=k\left(\frac{4 \pi \varepsilon_{0} \varepsilon_{r} \psi_{0}^{2} b}{A}\right)\right)^{w}\left(\frac{A}{6 \pi \eta b^{3} \dot{\gamma}}\right)^{x} \sum_{m=n /\left(1-z_{p}\right)}^{n} \frac{n !}{m !(n-m) !}\left[1-\left(1-\left(1-z_{p}\right) \theta\right)^{-2 w}\right]^{m}\left[\left(1-\left(1-z_{p}\right) \theta\right)^{-2 w}\right]^{n-m}
$$

where $\mathrm{z}_{\mathrm{p}}$ is a fitting parameter which was used to obtain fractional surface coverage from the surface charge measurements. This model was specifically developed for noncolloidal size particles and takes fluid mechanical and polymer adsorption parameters into account. The first term in parenthesis on right hand side shows the relative 
magnitude of double layer repulsion to van der Waals attraction. The second term signifies the effect of hydrodynamic forces. The summation term accounts for the surface coverage and is based on Deason's model. Unlike other models, this model also addresses the shear rate dependence of collision efficiency. One significant aspect of this work was that it studied the effect of both surface coverage and shear rate dependence of collision efficiency. As shown in the previous section, in case of shear-induced coagulation, dependency of collision efficiency is shear rate to the -0.18 power (see equation 2.28 ). Aunins' work showed that this dependency changes with the surface coverage. At high surface coverage, collision efficiency is much less dependent on shear rate than at low surface coverage [29]. From this it can be concluded that as the surface coverage changes so does the mechanism of agglomeration-from coagulation dominated at low surface coverage to bridging dominated at high surface coverage resulting in different shear rate dependence. In equation (3.8) exponent x governs the shear rate dependency, however, it does not show how it should change with the changing surface coverage.

\subsection{Agglomerate Size}

When a suspension is sheared, hydrodynamic forces that are responsible for bringing particles together to form agglomerates are also responsible for breaking up of the flocs. As the agglomerate size grows, agglomerate break up becomes increasingly significant and ultimately an equilibrium exists between agglomerate formation and agglomerate breakage, leading to the attainment of an equilibrium size.

Floc break up may occur by two mechanisms- surface erosion or floc-splitting. In the surface erosion mechanism, primary particles get removed from the outer surface of 
the agglomerate whereas in the floc-splitting mechanism, the agglomerate ruptures into several smaller size fragments.

An agglomerate has a porous structure composed of randomly packed spherical particles. The tensile strength of such an agglomerate is given by [30]:

$\sigma_{z}=\frac{9}{8}\left(\frac{1-\varepsilon_{A}}{\varepsilon_{A}}\right) \frac{F_{A}}{d^{2}}$

where, $\varepsilon_{\mathrm{A}}$ is the porosity of the agglomerate, $\mathrm{d}$ is agglomerate size and $\mathrm{F}_{\mathrm{A}}$ is the net attractive force binding particles together. In a flow field, agglomerates experience tensile as well as compressive hydrodynamic forces. For an agglomerate consisting of two spherical particles of size $\mathrm{b}$ and $\mathrm{b} \Lambda(0<\Lambda \leq 1)$, adhered together, the hydrodynamic force on the larger sphere is given by [31]:

$F_{h}=\pi \eta b \dot{\gamma}\left[h_{1}(\Lambda) \underline{E} \cdot \underline{n}+h_{2}(\Lambda) \underline{n} \underline{n} \cdot \underline{E} \cdot \underline{n}\right]$

where $\underline{E}$ is the rate of deformation tensor, $\underline{n}$ is the unit vector normal to plane of rupture. Also, $h_{1}(\Lambda)$ and $h_{2}(\Lambda)$ are functions of relative size of spheres. $h_{1}(\Lambda)+h_{2}(\Lambda)$ is maximum when both spheres have the same size. This implies that an agglomerate is most likely to rupture in two equal halves. The adhesive force at the rupture plane of area $\mathrm{S}$ is:

$$
F_{c}=\sigma S \underline{n}
$$

and the rupture of an agglomerate will occur when the hydrodynamic tension exceeds the force of adhesion, i.e.:

$\frac{F_{h} \cdot \underline{n}}{F_{c} \cdot \underline{n}} \geq 1$

In cases of shear flow and uniaxial extensional flow, hydrodynamic forces simplify to [32]: 


$$
F_{s h}=2.5 \pi \eta r_{a}^{2} \dot{\gamma}
$$

and

$$
F_{e x t}=5 \pi \eta r_{a}^{2} \dot{\gamma}_{e x t}
$$

where, $\mathrm{r}_{\mathrm{a}}$ is the agglomerate radius and $\dot{\gamma}_{\text {ext }}$ is the elongation rate. From equations (3.9), (3.11) and (3.12), the effect of agglomerate size on floc strength and hydrodynamic stress becomes apparent. Equation (3.9) indicates that the tensile stress is inversely proportional to square of agglomerate size which means that larger agglomerates are much weaker and thus easy to rupture. Equations (3.13)-(3.14) show that the hydrodynamic force increases as the square of agglomerate size and the rupture force is higher in an extensional flow field than in a shear flow field. This also indicates that at higher shear rate only smaller agglomerates will survive.

Sonntag and Russell [33] studied the breakage of colloidal polystyrene latex of $0.14 \mu \mathrm{m}$ diameter perikinetically coagulated with an electrolyte in $55.2 \%$ glycerin solution. Coagulated particles were subject to instantaneous high shear rate spikes to cause the breakage but to minimize the reagglomeration at the same time. The average size of the resulting fragments was measured and plotted against the shear stress. It was found that the mean radius of gyration cubed varied as $(\eta \dot{\gamma})^{-1.06}$.

Oles [34] studied the shear-induced agglomeration of $2.17 \mu \mathrm{m}$ diameter polystyrene latex particles in a laminar shear flow generated by a Coutte-flow device. It was found that increasing shear rate produced agglomerates of decreasing stable size.

Most of the floc breakage studies have been carried out in the turbulent flow region because practically encountered suspensions are usually aqueous and in the laminar flow region stresses generated are not high enough to break the agglomerates. In 
a turbulent flow field, floc breakage behavior depends on the intensity of the turbulence measured in terms of eddy size. If the eddy size is bigger than the agglomerate, it is considered that the agglomerate is completely encompassed in the eddy. Within that eddy, the agglomerate experiences laminar flow and only a viscous shear force. However, as the intensity of turbulence increases and the eddy size decreases, eddies move around the agglomerates. This fluctuating motion of eddies imparts shear, tensile and compressive stresses on the agglomerate and is more effective in causing the breakage. A general relationship for the maximum agglomerate in turbulent flow can be given as [35]:

$d_{F, \text { max }} \approx F_{A}^{l_{1}} d^{-l_{2}} E_{\text {turbulent }}^{-l_{3}}$

where, $\mathrm{E}_{\text {tutbulent }}$ is the average energy dissipation in turbulent flow. Exponents $1_{1}, l_{2}$ and $l_{3}$ depend on the size of agglomerates and eddies, and are determined experimentally, though some theoretical estimates are available [36].

Morphology of agglomerates plays a significant part if one attempts to theoretically determine the maximum agglomerate size and its breakage behavior. By morphology one means how the primary particles are packed together in an agglomerate and what is the shape of the agglomerate. As is evident from equation (3.9), the strength of an agglomerate depends on its porosity. A highly porous agglomerate is much easier to break than a densely packed one. Secondly, agglomerates are not uniform spherical bodies but rather irregular-shaped objects. Agglomerates tend to rupture at the weakest point which occurs wherever the number of contacts (or adhesive strength) between the particles at a cross-section is the least. When an agglomerate ruptures it breaks into several fragments. Glasgow and Hsu [37] studied the breakup of flocs by introducing individual flocs in a turbulent jet and taking the photographs of the breakage. It was 
found that the parent floc splits into several smaller flocs of varying size and shapes. This would suggest that breakup of agglomerates is not a deterministic process but rather a stochastic process. Pandya and Spielman [38] presented a model to simulate the floc breakage in an agitated suspension. In this model, it was assumed that when an aggregate ruptures, the particle size distribution of resulting daughter fragments can be given by a probability function such as a Gaussian distribution or a log-normal distribution. Mass balance for the breakage of an agglomerate in size range i, can be given as [39]:

$$
\frac{d w_{i}}{d t}=-S_{i} w_{i}+\sum_{j=1}^{i-1} B_{i j} S_{j} w_{j}
$$

Here $\mathrm{i}=1$ represents the largest agglomerate size. $\mathrm{S}_{\mathrm{i}}$ is the splitting frequency given by [40]:

$S_{i}=k_{s} w_{i}^{m}$

$\mathrm{k}_{\mathrm{s}}$ and $\mathrm{m}$ are constants. $\mathrm{S}_{\mathrm{i}}=0$, if $\mathrm{w}_{\mathrm{i}}<$ maximum stable size, i.e., only the agglomerates which are larger than the maximum stable floc size undergo breakage. Also, bigger agglomerates break much easily than smaller ones. $\mathrm{B}_{\mathrm{ij}}$ is the fraction of the fragments from agglomerate of size $\mathrm{j}$ which falls into class $\mathrm{i}$ and is determined by probability distribution function.

To model the floc breakage, the particle size distribution of a suspension undergoing shear flow, such as during agitation, is monitored with respect to time and data fitting coupled with mass balance is used to obtain the constants $\mathrm{k}_{\mathrm{s}}$ and $\mathrm{m}$.

At this point it is pertinent to point out that in practice, a real suspension system consists of particles that are polydispersed and the flow field causing the agglomeration and breakage is non-homogeneous such as obtained by stirring of suspensions in flocculation tanks by impellers. Because of these non-idealities, theoretical models 
developed for ideal systems do not adequately describe the real systems. Therefore, the approach that is usually followed is to model the agglomeration process by population balance models. In the simplest form, population balance model is a kinetic equation where the change in the number of particles of a given size is expressed in terms of gains and losses due to agglomeration and breakage. In a batch flocculator the ArgamanKaufman equation can be used [41]:

$$
\frac{N_{1}}{N_{2}}=\frac{K_{b}}{K_{a}} \dot{\gamma}+\left(1-\frac{K_{b}}{K_{a}} \dot{\gamma}\right) e^{-K_{b} \dot{x}}
$$

where, $N_{1}$ is number concentration of particles at time $t, N_{0}$ is initial concentration, $K_{a}$ is aggregation constant, $\mathrm{K}_{\mathrm{b}}$ is breakup constant and $\dot{\gamma}$ is shear rate. Both $\mathrm{K}_{\mathrm{a}}$ and $\mathrm{K}_{\mathrm{b}}$ are determined by data fitting.

In general, a floc population balance can be expressed as [42]:

$\frac{d N}{d t}=B_{a}-D_{a}+B_{b}-D_{b}$

Here, $\mathrm{B}_{\mathrm{a}}$ and $\mathrm{B}_{\mathrm{b}}$ are "birth" rates (gain) of flocs due to aggregation and floc breakage respectively, and $D_{a}$ and $D_{b}$ are "death" rates (loss) due to aggregation and breakage. In expanded form, a population balance model where agglomeration and floc breakage in a shear flow is occurring can be given as [43]:

$$
\frac{d N_{i}}{d t}=\frac{1}{2} \sum_{j+k=i} \alpha_{j k} \beta_{j k} N_{j} N_{k}-N_{i} \sum_{k=1}^{\max } \alpha_{i k} \beta_{i k} N_{k}-S_{i} N_{i}+\sum_{j=i}^{\max } b_{i j} S_{j} N_{j}
$$

In the above equation $\alpha$ is the collision efficiency and $\beta$ is the collision frequency as described in section 2.1. The first two terms on the right represent gain and loss respectively of particles in size class $\mathrm{i}$ due to agglomeration, while last two terms represent gain and loss due to floc breakage. During agglomeration, the particle size 
distribution is monitored with respect to time and differential equations are solved for $\alpha$ and $\beta$. A number of variations of population balance models exist in the literature where different forms of loss and gain terms are used (see for example [43], [44] and [45]).

\subsection{Parameters Governing Collision Efficiency and Agglomerate Size}

\subsubsection{Effect of Solvent}

Interaction between polymer and the solvent has a significant effect on the flocculation process. The Flory-Huggins theory is commonly used to describe the polymer-solvent interaction. This theory considers the change in the Gibb's free energy as the polymer is dissolved in the solvent. The solution is visualized as a two-dimensional lattice of $\mathrm{N}$ sites. Each site can be occupied by either a solvent molecule or a segment of polymer molecule. A long chain polymer molecule consists of $\mathrm{n}$ segments or monomer units. The Gibb's free energy is the combination of entropy of mixing $\left(\Delta S^{\mathrm{M}}\right)$ and the enthalpy of mixing $\left(\Delta \mathrm{H}^{\mathrm{M}}\right)$ :

$\Delta G^{M}=\Delta H^{M}-T \Delta S^{M}$

The entropy of mixing is obtained by considering the number of ways solvent molecules and the polymer segments can be placed in the three dimensional lattice. It is given by [46]:

$\Delta S^{M}=-k_{B}\left(n_{1} \ln v_{1}+n_{2} \ln v_{2}\right)$

where $\mathrm{n}_{1}$ and $\mathrm{n}_{2}$ are the number of molecules of solvent and polymers respectively, and $v_{1}$ and $v_{2}$ are volume fractions of solvent and polymers. The enthalpy of mixing is obtained by calculating the change in energy as a solvent molecule comes in contact with a polymer segment and it is given by [46]: 


$$
\Delta H^{M}=n_{1} v_{2} \chi k T
$$

The Flory-Huggins parameter $\chi$ signifies the interaction between polymer molecules and the solvent and is given by [46]:

$$
\chi=\frac{z \Delta e}{k T}
$$

where $\mathrm{z}$ is the coordination number and $\Delta \mathrm{e}$ is the change in energy.

If for a given polymer-solvent system $\chi$ is less than 0.5 , the solvent is considered a "good solvent", because in this case polymer-solvent interaction is favored over polymer- polymer interaction and polymer molecules remain dispersed in the solvent. On the other hand if $\chi$ is more than 0.5 , the opposite occurs and polymer-polymer interaction is favored and the polymer tends to precipitate out. Consider solid particles covered with a polymer, coming in contact with each other. If they are in a good solvent, polymer segments at the surface repel each other and particles do not flocculate and particles are considered sterically stabilized. However, if solvent quality is not good, polymer molecules associate with each other and flocculation ensues.

In the case of nonionic homopolymers such as polyethylene oxide (PEO), water is a good solvent. Therefore, in an aqueous system consisting only of solid particles and PEO, polymer does not adsorb on the particles and does not cause flocculation. The solvency of the medium can be changed by adding an electrolyte, by changing the temperature or by adding a non-solvent [47]. Cowell and Vincent [48] flocculated PEOstabilized polystyrene lattices by adding $\mathrm{MgSO}_{4}$ and raising the temperature of the suspension. The temperature at which flocculation occurs is known as the critical flocculation temperature. De Witt and van de Ven [49] studied the stability of PEO 
coated polystyrene lattices and found that the flocculation increases with the increasing amount of added $\mathrm{KCl}$. Increasing the temperature also increases the amount of PEO adsorbed by the particles. Therefore, the polymer bridging flocculation is more effective in a medium where the polymer-solvent interaction is not favored.

Quality of the solvent also has an effect on the maximum stable floc size as it affects the strength of the polymer bridges between the particles. Increasing the amount of electrolyte produces stronger flocs [50].

\subsubsection{Effect of Molecular Weight of the Polymer}

As mentioned earlier, when a polymer molecule adsorbs on the surface of the solid particles it acquires a conformation in the form of loops, trains and tails which extend into the medium which determines the thickness of the polymer layer. This effectively increases the hydrodynamic radius of the particles, especially in the case of colloidal particles where the polymer molecules and the particles may be of comparable size. Many studies have been carried out where the thickness of the adsorbed PEO polymer layer has been measured [49, 51-53]. It is found that the thickness increases with increasing molecular weight of the polymer. Also a high molecular weight polymer molecule covers more of the surface area of the particle than a low molecular weight polymer. Therefore, a high molecular weight polymer at low concentration is a more effective flocculant than a low molecular weight polymer [54]. Since the polymer increases the effective radius of the particles, it is also found that the difference between the collision efficiencies of homo- and heterocoagulation decreases [42]. 
The strength of agglomerates and maximum stable floc size are also influenced by the molecular weight of the polymer. Both adhesive strength and maximum floc size increase with increasing molecular weight of the polymer [35]. A bridge formed by a large polymer molecule between two particles has many more points of contact between them, thus more force is required to break them apart resulting in stronger and larger flocs. 


\section{Chapter 4. Theory}

\subsection{Development of Collision Efficiency Model}

As described in the chapter 3 , most of the models available to determine the collision efficiency, whether probabilistic or deterministic in their approach are directed more essentially towards flocculation of colloidal suspensions. A model is therefore required which can address the effects of hydrodynamic conditions and polymer adsorption on the collision efficiency of non-colloidal systems.

One way to approach this problem is to extend the calculation of trajectories to the case where the solid particle surface is not bare but carries adsorbed patches or a layer of polymer flocculant. In this case, a problem arises as the colliding particles come in close proximity to the extent where the flow of the dispersing liquid squeezing out of the space between two particles is hindered by the polymer molecules [55]. It is, therefore, not an easy task to model such a flow. Secondly, one would have to account for the orientation of bare and covered particle surfaces. In other words, it is important to know if the covered surface is approaching the bare surface of other particle or the bare surface is approaching the bare surface or if the covered surface is approaching the covered surface of a particle and how the rotation of particles along their axes affects the phenomenon. This makes the problem even more intractable.

In this work, rather than solving the equations derived from trajectory analysis, dimensional analysis has been used to formulate dimensionless groups that involve the various forces acting on the flocculating particles. This approach is similar to the one taken by Aunins [26] who suggested that collision efficiency could be expressed in terms of dimensionless numbers in the form of a general power law as: 


$$
\alpha=k C_{R}^{v} C_{A}^{x} C_{S}^{y}
$$

where, $C_{R}$ accounts for the contribution of electric double-layer repulsion, $C_{A}$ takes care of van der Waals attraction, and third term $\mathrm{C}_{\mathrm{S}}$ represents the contribution of steric interaction which can be either attractive or repulsive in nature.

Specific forms of $C_{R}$ and $C_{A}$ were obtained from the trajectory analysis of the coagulating colloids. According to van de Ven and Mason [12]:

$$
C_{R}=\frac{2 \varepsilon_{r} \varepsilon_{0} \psi_{0}^{2}}{3 \eta b^{2}}
$$

and,

$$
C_{A}=\frac{A}{36 \pi \eta \dot{\gamma b}^{3}}
$$

Here, electric double-layer repulsion and van der Waals attraction have been normalized with respect to the hydrodynamic force. Zeichner and Schowalter [8] proposed a slightly different, but equivalent, formulation:

$$
\begin{aligned}
& C_{R}=\frac{\varepsilon_{r} \varepsilon_{0} \psi_{0}^{2} b}{A} \\
& \text { and } \\
& C_{A}=\frac{A}{6 \pi \eta \dot{\gamma}^{3}}
\end{aligned}
$$

Here electric double-layer repulsion has been normalized with respect to van der Waals attraction and van der Waals attraction with respect to the hydrodynamic force. Aunins [26] used the Zeichner and Schowlater's form. Note that form of $C_{S}$ is not specified in the literature. In the present work, a form of $\mathrm{C}_{\mathrm{S}}$ has been derived and the 
parameters $C_{R}$ and $C_{A}$ have been modified to account for the presence of a polymer layer on the particle surface.

When collisions take place between two particles that each has a fraction $\theta$ of its surface covered with polymer, the following scenarios can be envisaged:

Case 1: The bare surface of one particle collides with the bare surface of the other particle as shown in Figure (4.1a). The probability of this kind of encounter taking place is $(1-\theta)^{2}$. In this case the total interaction force has contributions from both van der Waals attraction and electric double-layer repulsion. The collision efficiency between bare-bare surfaces is given by:

$\alpha_{B B}=k_{1} C_{A}^{x_{1}} C_{R}^{y_{1}}(1-\theta)^{2}$

Case 2: Figure (4.1b) depicts the situation where the covered surface of one particle collides with the bare surface of other particle. This is usually accompanied by polymer bridging and the probability of occurrence is $2 \theta(1-\theta)$. Here, the attractive force is the van der Waals force but, this must be modified to include the effect of the polymer layer in terms of modified Hamaker constant A'. The repulsion force is again due to the electric double-layer. The collision efficiency is given by:

$\alpha_{B C}=k_{2} C_{A^{\prime}}^{x_{2}} C_{R}^{y_{2}} 2 \theta(1-\theta)$

Case 3: When two surfaces, both of which are covered with polymer approach each other as shown in Figure (4.1c), the polymer layers overlap. This interaction is known as steric interaction and, depending on the nature of polymer-solvent interaction as discussed later, it can contribute either to attraction or repulsion. The probability of this 

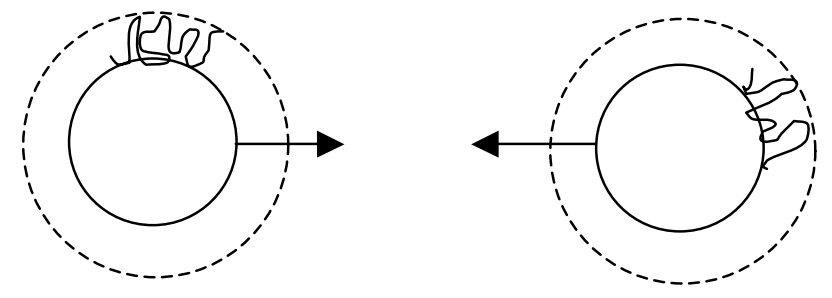

(a) Interaction between bare surfaces
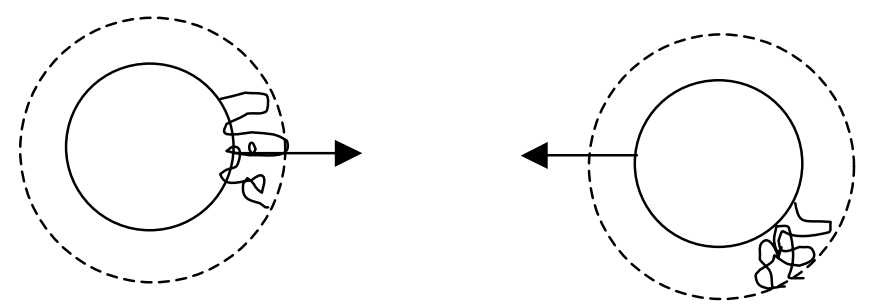

(b) Interaction between a covered and a bare surface


(c) Interaction between two covered surfaces

Figure 4.1. Possible interactions between the surfaces of partially covered solid particles. 
kind of interaction is given by $\theta^{2}$. Other contributing forces are a modified van der Waals force due the presence of polymer layers and the electric double-layer repulsion. Thus, in this case the collision efficiency is given by:

$\alpha_{C C}=k_{3} C_{A^{\prime \prime}}^{x_{3}} C_{R}^{y_{3}} C_{S}^{z_{3}} \theta^{2}$

In a process, where all three of the above-mentioned processes are occurring simultaneously, the total collision efficiency is given by:

$$
\begin{aligned}
& \alpha=\alpha_{B B}+\alpha_{B C}+\alpha_{C C} \\
& =k_{1} C_{A}^{x_{1}} C_{R}^{y_{1}}(1-\theta)^{2}+k_{2} C_{A^{\prime}}^{x_{2}} C_{R}^{y_{2}} 2 \theta(1-\theta)+k_{3} C_{A^{\prime}}^{x_{3}} C_{R}^{y_{3}} C_{S}^{z_{3}} \theta^{2}
\end{aligned}
$$

This is similar to the expression suggested by De Witt and van de Ven [49] for perikinetic agglomeration of particles:

$$
\alpha=\alpha_{B B}^{\prime}(1-\theta)^{2}+\alpha_{B C}^{\prime} 2 \theta(1-\theta)+\alpha_{C C}^{\prime} \theta^{2}
$$

where, $\alpha^{\prime}{ }_{\mathrm{BB}}, \alpha^{\prime}{ }_{\mathrm{BC}}, \alpha^{\prime}{ }_{\mathrm{CC}}$ are perikinetic counterparts of collision efficiency factors.

It should be pointed out that if one expressed the collision efficiency only as a function of fractional surface coverage as suggested by probabilistic models, the total collision efficiency, where all combinations of interactions between covered and bare surfaces result in agglomerate formation, would be equal to unity, i.e.:

$\alpha=(1-\theta)^{2}+2 \theta(1-\theta)+\theta^{2}=1$

This is not the case in practice because the hydrodynamic force tends to reduce the collision efficiency and depending on the shear rate, the collision efficiency will never be unity.

Now consider the following cases: 
(i) No polymer is present in the suspension, i.e., $\theta \rightarrow 0$, now equation (4.9) becomes:

$\alpha=k_{1} C_{A}^{x_{1}} C_{R}^{y_{1}}$

which is same as the collision efficiency when agglomeration takes place because of coagulation only.

(ii) The entire surface of the solid particle is completely covered with polymer, i.e., $\theta \rightarrow 1$. In this case, the collision efficiency becomes:

$\alpha=k_{3} C_{A^{\prime \prime}}^{x_{3}} C_{R}^{y_{3}} C_{S}^{z_{3}}$

(iii) If $0<\theta<1$, In this case, the optimum amount of polymer coverage needed for maximum collision efficiency is obtained by setting $d \alpha / d \theta$ equal to zero:

$$
\frac{d \alpha}{d \theta}=-2 k_{1} C_{A}^{x_{1}} C_{R}^{y_{1}}(1-\theta)+2 k_{2} C_{A^{\prime}}^{x_{2}} C_{R}^{y_{2}}(1-2 \theta)+2 k_{3} C_{A^{\prime \prime}}^{x_{3}} C_{R}^{y_{3}} C_{S}^{z_{3}} \theta=0
$$

on rearranging:

$\theta_{\text {optimum }}=\frac{k_{1} C_{A}^{x_{1}} C_{R}^{y_{1}}-k_{2} C_{A^{\prime}}^{x_{2}} C_{R}^{y_{2}}}{k_{3} C_{A^{\prime \prime}}^{x_{3}} C_{R}^{y_{3}} C_{S}^{z_{3}}+k_{1} C_{A}^{x_{1}} C_{R}^{y_{1}}-2 k_{2} C_{A^{\prime}}^{x_{2}} C_{R}^{y_{2}}}$

In this work, the amount of added polymer is such that it significantly exceeds the amount needed for monolayer coverage of the particle surface. In addition, adsorption experiments suggested that at the concentration level of added polymer, all the polymer had adsorbed on particles and none remained in the solvent. Therefore, it was assumed that the surface of the particles was totally covered with polymer, i.e., $\theta=1$. Hence, the collision efficiency should be given by equation (4.13). The next step is to obtain expressions for the dimensionless parameters $\mathrm{C}_{\mathrm{A}}, \mathrm{C}_{\mathrm{R}}$ and $\mathrm{C}_{\mathrm{S}}$. 


\subsubsection{Derivation of the Expression for $\mathrm{C}_{\mathrm{A}}$ "}

Consider two spherical solid particles approaching each other along the line of their centers as shown in Figure (4.2). The radius of each particle is b, the thickness of the adsorbed polymer layer on each particle is $\delta$ and the closest distance between the surfaces of particles is s. The van der Waals attraction energy for two spherical particles covered with a polymer layer is given by [56]:

$V_{A}=-\frac{1}{12}\left[\left(A_{0}^{1 / 2}-A_{2}^{1 / 2}\right)^{2} \frac{b+\delta}{s-2 \delta}+\left(A_{2}^{1 / 2}-A_{1}^{1 / 2}\right)^{2} \frac{b}{s}+4\left(A_{0}^{1 / 2}-A_{2}^{1 / 2}\right)\left(A_{2}^{1 / 2}-A_{1}^{1 / 2}\right) \frac{b+\delta}{(s-\delta)\left(2+\frac{\delta}{b}\right)}\right]$

where, $\mathrm{A}_{0}, \mathrm{~A}_{1}$ and $\mathrm{A}_{2}$ are the Hamaker constants for solvent, particle and polymer respectively. Here the negative sign indicates that this energy is attractive in nature. The corresponding force is obtained by differentiating this equation with respect to distance 's':

$F=-\frac{d V_{A}}{d s}$

$=-\frac{1}{12}\left[\left(A_{0}^{1 / 2}-A_{2}^{1 / 2}\right)^{2} \frac{b+\delta}{(s-2 \delta)^{2}}+\left(A_{2}^{1 / 2}-A_{1}^{1 / 2}\right)^{2} \frac{b}{s^{2}}+4\left(A_{0}^{1 / 2}-A_{2}^{1 / 2}\right)\left(A_{2}^{1 / 2}-A_{1}^{1 / 2}\right) \frac{b+\delta}{(s-\delta)^{2}\left(2+\frac{\delta}{b}\right)}\right]$

On substituting $\mathrm{s}^{*}=\mathrm{s} / \delta$ and rearranging, one gets: 


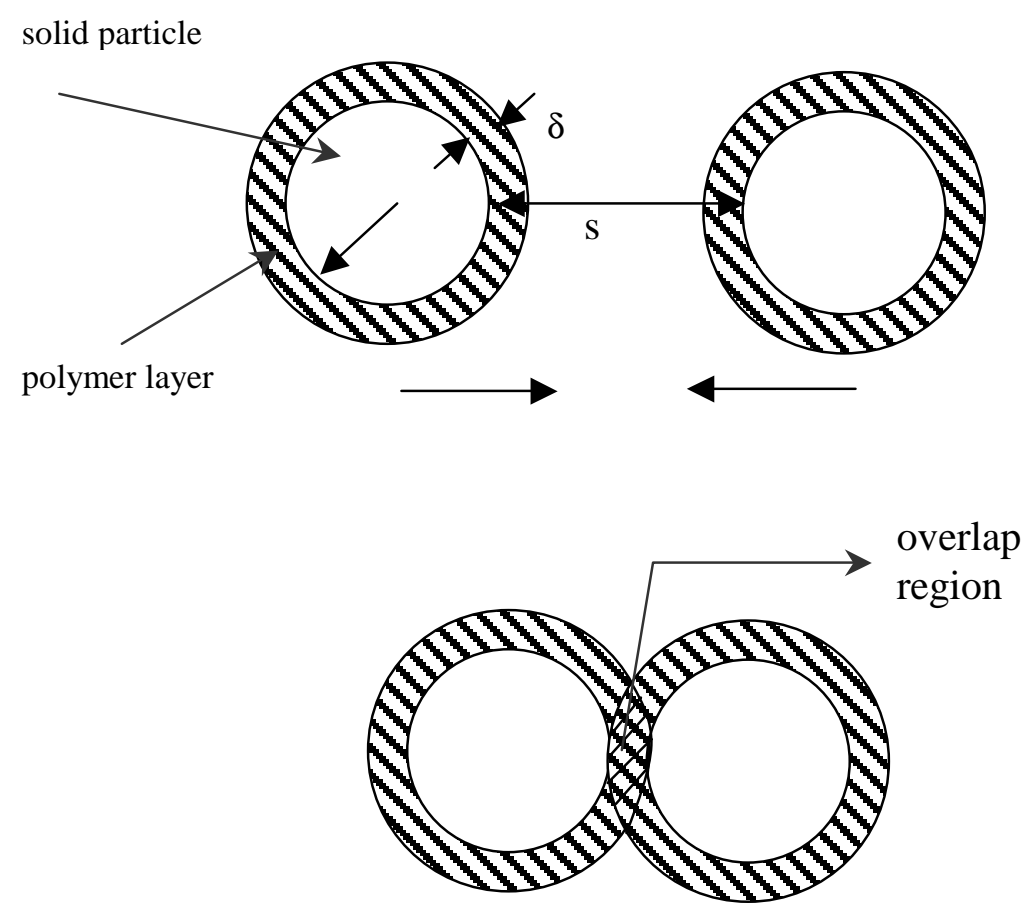

Figure 4.2. Overlapping of adsorbed polymer layer as particles approach each other. 


$$
F_{A}=-\left[\frac{\left(A_{0}^{1 / 2}-A_{2}^{1 / 2}\right)\left(A_{2}^{1 / 2}-A_{1}^{1 / 2}\right)(b+\delta)}{12 \delta^{2}}\right]\left[\frac{\left(A_{0}^{1 / 2}-A_{2}^{1 / 2}\right)}{\left(A_{2}^{1 / 2}-A_{1}^{1 / 2}\right)} \frac{1}{\left(s^{*}-2\right)}+\frac{\left(A_{2}^{1 / 2}-A_{1}^{1 / 2}\right)}{\left(A_{0}^{1 / 2}-A_{2}^{1 / 2}\right)} \frac{b}{(b+\delta) s^{*}}+4 \frac{1}{\left(s^{*}-1\right)\left(2+\frac{\delta}{b}\right)}\right]
$$

The term in the first square brackets gives the magnitude of the van der Waals attraction force. This must be scaled with respect to the hydrodynamic force $3 \pi \eta \dot{j} b^{2}$ which tends to oppose the attractive force. Thus the van der Waals dimensionless parameter is:

$$
C_{A^{n}}=\frac{\left(A_{0}^{1 / 2}-A_{2}^{1 / 2}\right)\left(A_{2}^{1 / 2}-A_{1}^{1 / 2}\right)(b+\delta)}{36 \pi \eta \dot{\gamma b}^{2} \delta^{2}}
$$

\subsubsection{Derivation of the Expression for $C_{R}$}

The electric double-layer repulsion energy is given by [9]:

$$
V_{R}=2 \pi \varepsilon_{r} \varepsilon_{0} b \psi_{0}^{2} \ln \left(1+e^{-\kappa s}\right)
$$

where $\kappa^{-1}$ is the electric double-layer thickness. The force of repulsion is given by:

$$
F_{R}=-\frac{d V_{R}}{d s}=2 \pi \varepsilon_{R} \varepsilon_{0} \psi_{0}^{2}(\kappa b) \frac{e^{-\kappa s}}{1+e^{-\kappa s}}
$$

Again on substituting s* $=\mathrm{s} / \delta$ and rearranging:

$$
F_{R}=\left[2 \pi \varepsilon_{R} \varepsilon_{0} \psi_{0}^{2}(\kappa b)\right]\left[\frac{e^{-(\kappa \delta) s^{*}}}{1+e^{-(\kappa \delta) s^{*}}}\right]
$$

If this is scaled with respect to the van der Waals attractive force $\approx \frac{\left(A_{0}^{1 / 2}-A_{2}^{1 / 2}\right)\left(A_{2}^{1 / 2}-A_{1}^{1 / 2}\right)}{\delta}$ one obtains the dimensionless parameter:

$C_{R}=\left[\frac{2 \pi \varepsilon_{r} \varepsilon_{0} \psi_{0}^{2}(\kappa b) \delta}{\left(A_{0}^{1 / 2}-A_{2}^{1 / 2}\right)\left(A_{2}^{1 / 2}-A_{1}^{1 / 2}\right)}\right]$ 


\subsubsection{Derivation of the Expression for $\mathrm{C}_{S}$}

When two polymer-covered solid particles come in contact with each other, their polymer layers overlap. The concentration of polymer increases in the overlap region. If the polymer segments repel each other, the particles are pushed back and agglomeration does not take place; this is called steric stabilization. However, if polymer segments attract each other; the layers completely overlap. The potential energy for this kind of interaction is given by [57]:

$V_{s}=\frac{4 \pi k_{B} T c^{2}\left(\frac{1}{2}-\chi\right)}{3 V_{1} \rho_{2}^{2}}\left[\left(\delta-\frac{s}{2}\right)^{2}\left(3 b+2 \delta+\frac{s}{2}\right)\right]$

where, $\mathrm{k}_{\mathrm{B}}$ is Boltzmann's constant, $\mathrm{c}$ is the concentration of polymer in the polymer layer, $\mathrm{V}_{1}$ is the molecular volume of solvent and $\rho_{2}$ is the density of polymer. Whether the interaction results in attraction or repulsion depends on the magnitude of the FloryHuggins parameter $\chi$. If $\chi>1 / 2$, layers will attract and if $\chi<1 / 2$, layers will repel each other. Here again the force is obtained by differentiating the potential with respect to s. Substituting $\mathrm{s}^{*}=\mathrm{s} / \delta$ in the resulting expression and rearranging gives:

$F_{s}=\frac{2 \pi k_{B} T c^{2}\left(\frac{1}{2}-\chi\right) \delta^{2}}{V_{1} \rho_{2}^{2}}\left[\frac{s^{* 2}}{4}-\frac{b}{\delta}-1\right]$

When this is scaled with respect to the hydrodynamic force, one obtains the dimensionless steric interaction parameter as:

$C_{s}=\frac{2 k_{B} T c^{2}\left|\left(\frac{1}{2}-\chi\right)\right| \delta^{2}}{3 V_{1} \rho_{2}^{2} \eta \dot{b} b^{2}}$

The collision efficiency in case of total polymer coverage can now be written as: 


$$
\alpha=k_{3}\left[\frac{\left(A_{0}^{1 / 2}-A_{2}^{1 / 2}\right)\left(A_{2}^{1 / 2}-A_{1}^{1 / 2}\right)}{36 \pi \eta \dot{\gamma} b^{2} \delta^{2}}\right]^{x_{3}}\left[\frac{2 \pi \varepsilon_{r} \varepsilon_{0} \psi_{0}^{2}(\kappa b) \delta}{\left(A_{0}^{1 / 2}-A_{2}^{1 / 2}\right)\left(A_{2}^{1 / 2}-A_{1}^{1 / 2}\right)}\right]^{y_{3}}\left[\frac{2 k_{B} T c^{2}\left|\left(\frac{1}{2}-\chi\right)\right| \delta^{2}}{3 V_{1} \rho_{2}^{2} \eta \dot{\gamma} b^{2}}\right]^{z_{3}}
$$

The variables in the brackets depend on the physicochemical characteristics of the suspension and the shear rate. Exponents $\mathrm{x}_{3}, \mathrm{y}_{3}, \mathrm{z}_{3}$ and the constant $\mathrm{k}_{3}$ must be determined by experimentally obtained values of $\alpha$ at different conditions of flocculation using non-linear regression analysis. Once the values of exponents are known it would be possible to see how the flocculation conditions affect the collision efficiency.

For a given suspension, the dispersion medium and the size of the particles are fixed. Thus, the viscosity and electrical permittivity of the medium would be invariant. Once type of polymer flocculant is chosen, Hamaker constant and Flory-Huggins parameter would also be fixed. If the electrolyte concentration and $\mathrm{pH}$ of the suspension are kept constant, the surface potential and electric double-layer thickness would also remain unchanged in case of nonionic flocculant. Therefore, besides the rate of shearing, other important variables affecting the collision efficiency would be the adsorbed layer thickness and the concentration of polymer in the adsorbed layer. A method to estimate their values is described in the next chapter. 


\section{Chapter 5. Experimental Details}

\subsection{Materials Used}

A model suspension was designed keeping in view the objective of this work which was to study the shear-induced agglomeration of a non-colloidal suspension by polymer bridging in a laminar shear flow. The model suspension consisted of neutrally-buoyant spherical glass beads dispersed in an aqueous glycerin solution. High molecular weight polyethylene oxide was used as the flocculating agent.

\subsubsection{Solid Particles}

The glass beads were obtained from Potters Industries, Carlstradt, NJ. These are hollow spheres with density equal to $1.12 \mathrm{~g} / \mathrm{cc}$ unlike the solid glass beads which have a density of about $2.6 \mathrm{~g} / \mathrm{cc}$. Matched density particles are necessary to minimize buoyancy effects. Otherwise as the floc size increases, the flocs tend to settle and do not remain in the flow field. The average diameter of the particles as obtained was $11.2 \mu \mathrm{m}$, and there was a wide distribution of sizes. A sonic sifter was used to remove the fraction which was above $10 \mu \mathrm{m}$. The resulting fraction had an average diameter of $4.9 \mu \mathrm{m}$. The particle size distributions of original and sifted particles are shown in Figure (5.1).

\subsubsection{Dispersion Medium}

The dispersion medium was glycerin (obtained from Aldrich, Milwaukee, WI) diluted with distilled water. Glycerin is a non-solvent for PEO [58] and therefore it promotes adsorption of PEO on glass beads and causes flocculation of the particles. 




Figure 5.1. Particle size distributions for original and sieved hollow spherical glass beads. 
The density of pure glycerin is $1.26 \mathrm{~g} / \mathrm{cc}$. Also, glycerin is a high viscosity medium, which helps in obtaining high shear stress even at low shear rates which are associated with laminar flow. High shear stresses are necessary to cause floc breakage because, as mentioned earlier, flocs formed by polymer bridging are much stronger than those formed by coagulation. Most of the earlier studies on flocculation in the literature have been performed in the turbulent flow region where stresses are high enough to break up the agglomerates.

\subsubsection{Polymer Flocculant}

Polyethylene oxide was used as the flocculant in this study. It is a non-ionic watersoluble polymer. PEO has been studied very widely as a flocculating agent over the years and lot of information is available in the literature about its behavior (see for example [58]). PEO is a homopolymer and its molecules are made up of repeating units of ethylene and oxygen molecules:

$$
\left[-\mathrm{CH}_{2}-\mathrm{CH}_{2}-\mathrm{O}-\right]_{n}
$$

The polymer is soluble in water at room temperature in all proportions. However, it shows an inverse solubility-temperature relationship. As the temperature is increased to near the boiling point, polymer precipitates out [58].

PEO samples of molecular weight ranging from 100,000 to $5 \times 10^{6}$ were procured from Union Carbide and Polysciences Inc and were used as obtained. The polymer as obtained is a flaky white powder. Though, PEO is soluble in cold water, it is not easy to dissolve- the lower molecular weight polymer being most difficult to dissolve. When PEO is added to water, it readily absorbs the water, and particles adhere together to form 
a gel like clump which takes prolonged stirring-time, of the order of several days, to dissolve. In case of lower molecular weight polymer even prolonged stirring failed to give a clear completely dissolved solution. In order to facilitate the preparation of polymer solution, advantage is taken of the fact that PEO is insoluble in boiling water but soluble at lower temperatures. Hence, to make the aqueous solutions, a small amount of distilled water was initially brought to boil, and then the weighed amount of PEO was sifted into the boiling water while stirring. Then the solution was removed from the heat source and the remainder of cold water was added while stirring was maintained. This caused the temperature to cool down and the dissolution to occur. Stirring was carried out for a further about 12 hours to obtain a clear solution.

Even the above-described method failed to give clear solutions of low molecular weight PEO of 100,000-400,000 MW. To prepare these solutions a different method was used. The weighed amount of PEO was first wetted with very small amount of Acetone and then water was added while stirring. This method gave a clear uniform solution. Aqueous solutions of different concentrations were prepared and used within a week of preparation.

\subsection{Suspension Preparation}

To prepare the suspension, a known amount of glass beads was first dispersed in pure glycerin. Then a measured amount of an aqueous solution of PEO was added and quickly dispersed by stirring with a glass rod. The concentration and volume of the aqueous solution of PEO added were determined by the final concentration of polymer and water needed in the suspension. The method of polymer addition has a significant effect on the 
kinetics of agglomeration. Whether polymer solution is added in a single dose or in multiple doses influences the final agglomerate size [59]. Therefore, it was necessary that the method of polymer addition be kept same for all experiments. Here the polymer solution was added in a single dose. Fresh suspension was prepared each time for each experiment and was used immediately upon preparation. The quantities of various components are described as required in later sections.

\subsection{Shearing of the Suspension}

The shear-flow experiments were carried out using a cone and plate device. The cone was $8 \mathrm{~cm}$ in diameter and cone angles of $2^{\circ}$ and $4^{\circ}$ were available. A motor with a speed controller was attached to the cone, and shear rates of $0-380 \mathrm{~s}^{-1}$ could be obtained with this setup. The plate was maintained at a constant temperature by circulating constant temperature water through it using a Fisher-Scientific Isotemp water circulator. To minimize the effect of humidity, a nitrogen gas blanket was provided around the cone. This was done using a perforated plastic tube that was placed around the gap between cone and plate through which high-pressure nitrogen gas was passed. A schematic diagram of the arrangement is shown in Figure (5.2).

To shear the suspension a small amount $(\approx 8 \mathrm{ml})$ of the fresh suspension was placed on the plate and then the rotating cone was lowered on it. After a predetermined time, the cone was lifted slowly and a few drops of sample were withdrawn with the help of a wide-mouth dropper for subsequent image analysis. 


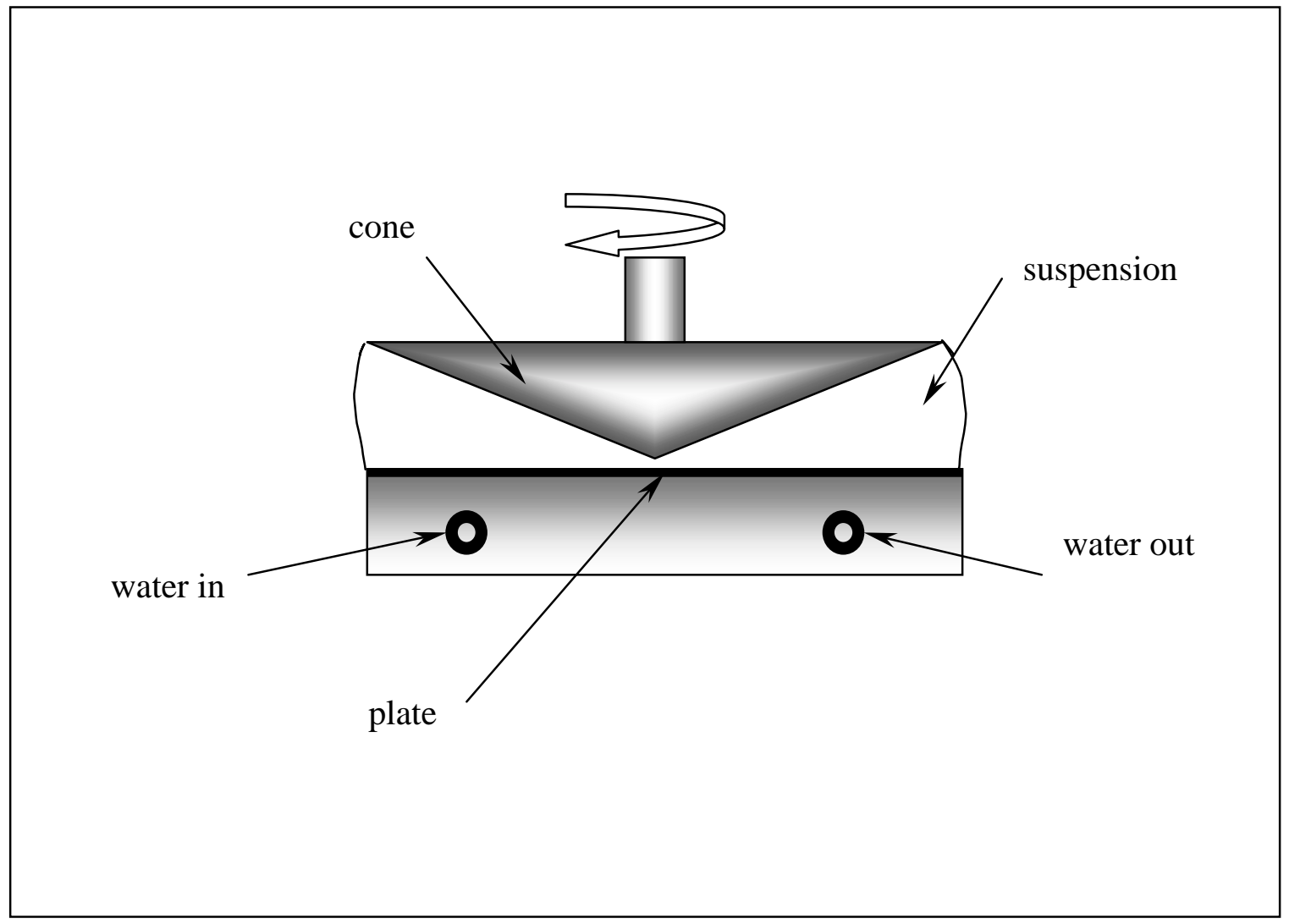

Figure 5.2. Schematic of the cone and plate device used for shearing the suspension. 


\subsection{Image Analysis}

Image analysis was used to count the number of agglomerates and to determine the size of the agglomerates. A small amount of the sheared suspension was placed in a transparent rectangular observation cell so that the agglomerates remained in the freefloating state and did not undergo distortion in shape and size. This also ensured that agglomerates remained randomly distributed through out the suspension.

A Micromaster phase-contrast microscope fitted with a digital camera was used to capture the images of the agglomerates. Initially when agglomerates were of smaller size higher magnification (10X) was used, but when agglomerates grew much bigger and became fewer in number, a lower magnification (4X) was used. "Scion Image" image analysis software was used to count the number of agglomerates and measure their projected area and perimeter. The microscope was focused at one focal plane and all the pictures were taken at that level. Several frames were captured for later analysis. When counting particles only those particles were included which were in focus. Out of focus particles were removed digitally from the images. Since only the relative count of the particles $\left(\mathrm{N} / \mathrm{N}_{0}\right)$ was required, it was not critical to establish the exact depth of focus for each experiment [60]. From the projected area of the agglomerate, its diameter can be calculated. Average agglomerate size was calculated as the number average diameter.

Before carrying out the procedure described above, calibration of the images was required. To do this, an image of a slide which had 300 equi-spaced lines per inch etched on it was captured and then the real distance of the lines was measured in terms of the number of pixels. Calibration was carried out for both magnifications. The calibration 
was further confirmed by measuring the diameter of commercially obtained spherical particles. The particle size comparison is shown in Table (5.1):

Table 5.1. Comparison of Sizes of Standard Spherical Particles

\begin{tabular}{|l|c|c|c|}
\cline { 2 - 4 } \multicolumn{1}{c|}{} & $\begin{array}{c}\text { Reported by } \\
\text { supplier }\end{array}$ & Measured & Measured \\
& at 10X & at 4X \\
\hline Average diameter $(\mu \mathrm{m})$ & 3.7 & 25.4 & 25.3 \\
\hline Standard deviation $(\mu \mathrm{m})$ & 3.9 & 3.18 & 3.92 \\
\hline
\end{tabular}

\subsection{Estimation of Flory-Huggins Parameter and Polymer Molecule Size}

Flory-Huggins parameter and the polymer molecule size are required to establish the interaction of PEO molecules in the mixed solvent of glycerin and water and to estimate the adsorption characteristics of PEO. Intrinsic viscosity measurements were carried out to experimentally determine the Flory-Huggins parameter and the radius of gyration of PEO molecules in the glycerin-water mixed solvent.

As mentioned earlier glycerin is a non-solvent for PEO and water is a good solvent. Thus a solution of $90 \%$ glycerin and $10 \%$ water by volume can be expected to have solvent properties for PEO which would lie somewhere in between. The intrinsic viscosity method was used to determine the thermodynamic properties of polymers in mixed solvents. This was based on similar work described in the literature; for example, Dondos and Benoit [61] measured properties of polystyrene and poly(2-vinylpyridine) in a series of mixed solvents which consisted of one solvent and one non-solvent in different proportions. 
The mean square end-to-end distance $\left\langle\mathrm{r}^{2}\right\rangle$ of a polymer molecule in a solution is given by following relation [62]:

$$
\left\langle r^{2}\right\rangle=\left\langle r^{2}\right\rangle_{0} \xi^{2}
$$

where $\left\langle\mathrm{r}^{2}\right\rangle_{0}$ is the unperturbed mean-square end-to-end distance and $\xi$ is the expansion factor. The unperturbed mean-square end-to-end distance is the mean square of polymer molecule dimensions in the solution at theta conditions and $\xi$ is the expansion factor which signifies the tendency of polymer molecules to shrink or elongate with respect to $\Theta$ conditions. For a very good solvent, $\xi$ is slightly less than 2 and for very poor solvent, slightly less than 1 [63]. It is given by [62]:

$$
\xi^{3}=\frac{[\eta]}{[\eta]_{\Theta}}
$$

where $[\eta]$ is the intrinsic viscosity of PEO in glycerin-water mixture at conditions under study and is determined by viscometric measurements as described later in this section. $[\eta]_{\Theta}$ is the intrinsic viscosity at $\Theta$ conditions which is given by:

$$
[\eta]_{\Theta}=K_{\Theta} M_{w}^{1 / 2}
$$

In order to determine $\mathrm{K}_{\Theta}$, Berry's equation was used [64]:

$$
\left(\frac{[\eta]}{M_{w}^{0.5}}\right)^{0.5}=K_{\Theta}^{0.5}+\Delta \frac{M_{w}}{[\eta]}
$$

where $\Delta$ is a constant. By plotting $\left(\frac{[\eta]}{M_{w}^{0.5}}\right)^{0.5}$ vs. $\frac{M_{w}}{[\eta]}$ a straight line is obtained whose slope is given by $\Delta$ and intercept is given by $K_{\Theta}^{0.5}$. Thus experiments were performed to measure the intrinsic viscosity of different molecular weight polymers. 
Cannon-Fenske capillary viscometers were used to measure the intrinsic viscosity of PEO of molecular weight 100,000 and 200,000 at $25^{\circ} \mathrm{C}$. A series of PEO solutions of decreasing concentration were prepared and their shear viscosity measured. Then for each molecular weight fraction, $\frac{1}{c}\left[\frac{\eta}{\eta_{0}}-1\right]$ was plotted against $\mathrm{c}$, where $\mathrm{c}$ is the PEO concentration in $\mathrm{gm} / \mathrm{dl}, \eta_{0}$ is the viscosity of the solvent and $\eta$ is the viscosity of the solution at concentration c. Intrinsic viscosity was obtained by extrapolating the graph to $\mathrm{c}=0$. This is shown in Figure (5.3) with the intercept. Each point is an average of two repeat experiments and a straight line was fitted to obtain the intercept.

Now using equation (5.4) and intrinsic viscosity measurements $\mathrm{K}_{\Theta}$ was obtained to be $1.34 \times 10^{-4}(\mathrm{dl} / \mathrm{gm})(\mathrm{mol} / \mathrm{gm})^{0.5}$ and using equations (5.2) and (5.3) intrinsic viscosity at $\Theta$ conditions and expansion factors were calculated and are given in Table (5.2).

Table 5.2. Measured Intrinsic Viscosity at $\Theta$ Conditions and the Expansion Factor

\begin{tabular}{|c|c|c|}
\hline Molecular Weight & {$[\eta]_{\Theta}(\mathbf{d} \mathbf{l} / \mathbf{g m})$} & $\xi$ \\
\hline 100,000 & $4.241 \times 10^{-2}$ & 0.958 \\
\hline 200,000 & $5.998 \times 10^{-2}$ & 0.936 \\
\hline
\end{tabular}

Values of expansion factors are less than 1, which signify that polymer molecules shrink in size and as expected the glycerin-water mixture forms a "bad" solvent for PEO. The next step is to estimate the molecular size of the polymer and for that, the following relation is used:

$$
\left\langle r_{0}^{2}\right\rangle^{3 / 2}=\frac{[\eta]_{\Theta} M_{w}}{\phi_{F}}
$$


where $\phi_{\mathrm{F}}$ is Flory's constant and is equal to $2.1 \times 10^{23}$ [62] together with:

$\left\langle r^{2}\right\rangle=\left\langle r_{0}^{2}\right\rangle \xi^{2}$

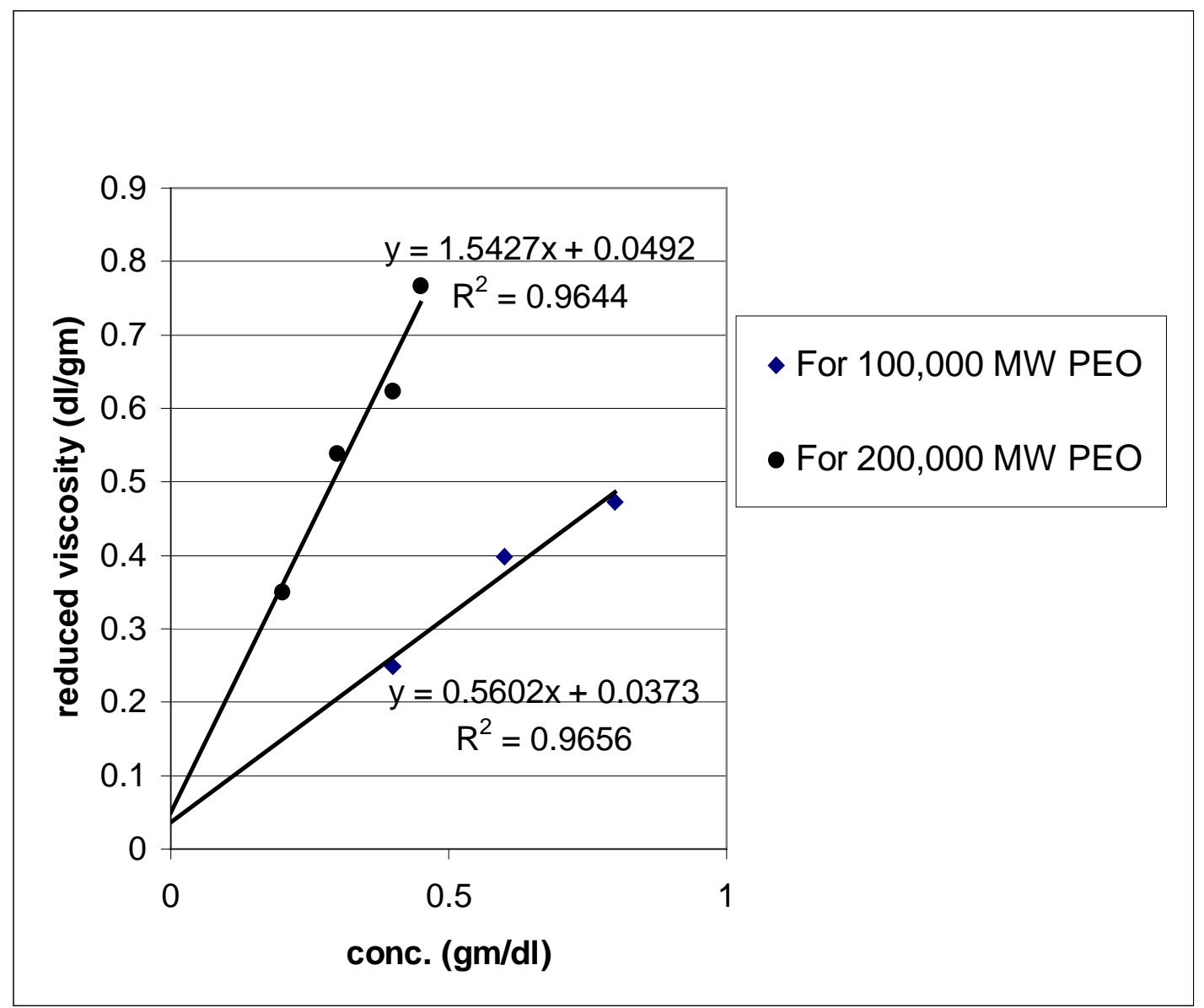

Figure 5.3. Reduced viscosity versus concentration of PEO to determine the intrinsic viscosity. 
give the mean square end-to-end distance for 100,000 and 200,000 MW PEO. To estimate the molecular size of higher molecular weight PEO it was assumed that $\langle\mathrm{r}\rangle \propto$ $\mathrm{M}^{1 / 2}$. From the mean square end-to-end distance, radius of gyration was calculated from [62]:

$$
\left\langle R_{g}\right\rangle^{2}=\frac{\langle r\rangle^{2}}{6}
$$

These values are tabulated below:

Table 5.3. Size of PEO Molecules in the Solvent

\begin{tabular}{|l|l|l|}
\hline Molecular weight & $\langle\mathbf{r}\rangle(\mathbf{n m})$ & $\left\langle\mathbf{R}_{\mathbf{g}}\right\rangle(\mathbf{n m})$ \\
\hline 100,000 & 12.11 & 4.94 (exp.) \\
\hline 200,000 & 16.73 & 6.82 (exp.) \\
\hline $1,000,000$ & 37.95 & 15.49 (est.) \\
\hline $5,000,000$ & 85.63 & 34.95 (est.) \\
\hline
\end{tabular}

In order to estimate the Flory-Huggins interaction parameter $\chi$, the following relation was used [62]:

$$
\xi^{5}-\xi^{3}=2 C_{M}\left(\frac{1}{2}-\chi\right) M_{w}^{1 / 2}
$$

where $C_{M}=\left(\frac{27}{2^{5 / 2} \pi^{3 / 2}}\right)\left(\frac{\bar{v}_{2}^{2}}{N_{A} V_{1}}\right)\left(\frac{M_{w}}{\left\langle r_{0}^{2}\right\rangle}\right)^{3 / 2}$. Here $\mathrm{V}_{1}$ is the molar volume of solvent $\left(=56.08 \mathrm{~cm}^{3} / \mathrm{mole}\right)$ and $\bar{v}_{2}$ is the specific volume of PEO $\left(=0.9 \mathrm{~cm}^{3} / \mathrm{gm}\right)$. Estimates of $\chi$ are given below: 


\section{Table 5.4. Estimation of Flory-Huggins Parameter}

\begin{tabular}{|l|l|l|}
\hline Molecular weight & 100,000 & 200,000 \\
\hline$\chi$ & 0.5011 & 0.50035 \\
\hline
\end{tabular}

That the Flory-Huggins parameter is greater than 0.5 signifies the fact that glycerinwater mixed solvent is a bad solvent for PEO. It was not possible to measure the intrinsic viscosity of PEO of molecular weight more than 200,000. The reason being, at low concentrations there was not any measurable change in the viscosity of solutions whereas at high concentrations, PEO precipitated out. Therefore, for calculation purposes $\chi$ was taken to be 0.50035 . It should be noted that $\chi$ would always be greater than 0.5 as long as expansion factor is less than 1. Furthermore, exact value will also depend on the molar volume of solvent and specific volume of PEO as evidenced by equation (5.8). In literature $\chi$ is usually reported up to three decimal places (see reference 47 for example). Here it has been reported up to four decimal places. Thus, the estimate of value of FloryHuggins parameter seems reasonable for calculation purposes.

\subsection{Adsorption of PEO}

As mentioned earlier, the collision efficiency depends on the fractional coverage $(\theta)$ of the particle surface by the polymer which is given by:

$$
\theta=\Gamma / \Gamma_{\max }
$$

where, $\Gamma$ is the amount of polymer adsorbed $\left(\mathrm{mg} / \mathrm{m}^{2}\right)$ at a given concentration and $\Gamma_{\max }$ is the amount adsorbed $\left(\mathrm{mg} / \mathrm{m}^{2}\right)$ at the saturation coverage of the particle surface. To obtain $\Gamma_{\max }$ it is necessary to obtain the adsorption isotherm of the polymer. The adsorption isotherm is established by determining the amount of the polymer adsorbed by the 


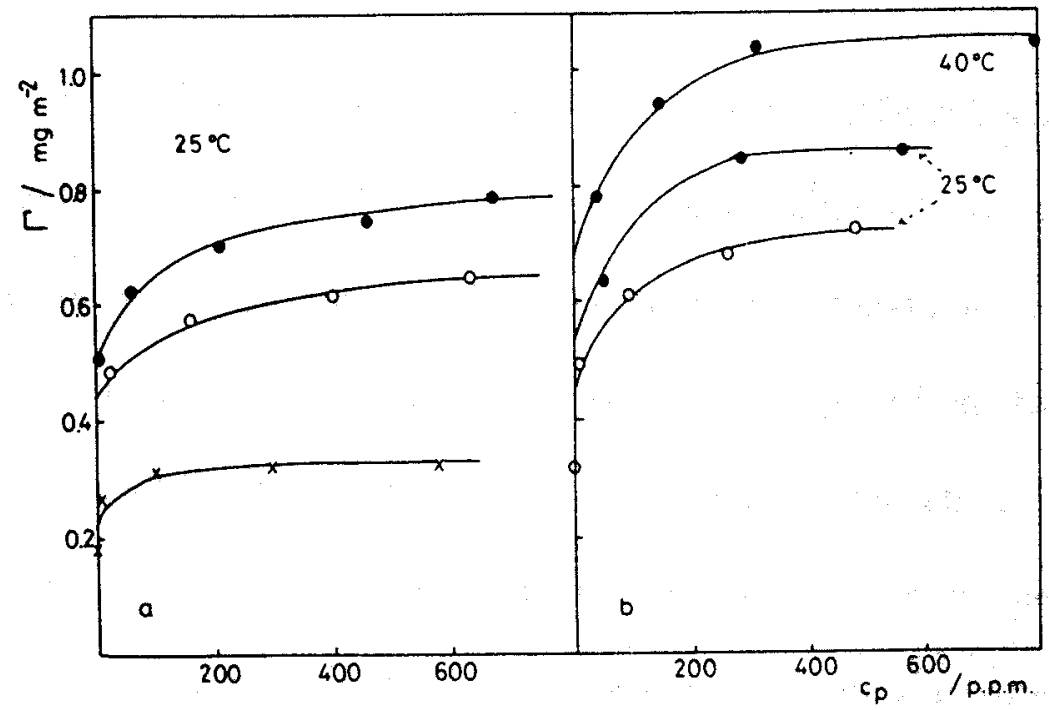

Figure 5.4. Adsorption isotherms for PEO on polystyrene. Absorbed amount $(\Gamma)$ vs. equilibrium polymer concentration $\left(c_{p}\right)$ x PEO 1500, o PEO 20,000, • PEO 300,000; (a) from water at $25^{\circ} \mathrm{C}$; (b) from $0.26 \mathrm{~mol} / 1 \mathrm{Mg} \mathrm{SO}_{4}$ solution at $25^{\circ} \mathrm{C}$ and $40^{\circ} \mathrm{C}$ (from Ref. [48]). 
particles at a given concentration of added polymer. For illustration, an adsorption isotherm for PEO on polystyrene latex suspension is shown in Figure 5.4. As can be seen with increasing amount of added polymer, more and more polymer adsorbs on the particles and then it reaches a plateau and no further adsorption takes place. The amount adsorbed corresponding to the plateau region is $\Gamma_{\max }$. The amount adsorbed $(\Gamma)$ at any other concentration can also be read from the isotherm and thus $\theta$ can be determined.

To determine the adsorption isotherm of PEO on glass beads in aqueous glycerin, the glass beads were first dispersed in the glycerin and then a known amount of the aqueous PEO solution was added and stirred over night with the help of a magnetic stirrer. Then the suspension was centrifuged to separate the particles. The supernatant was separated and analyzed for the remaining concentration of the PEO. Usually, the polymer concentration is in the range of few $\mathrm{mg} / \mathrm{lt}$. A turbidimetric method developed by Attia and Rubio [65] was used to determine the PEO concentration. If PEO solution is added to a solution of tannic acid and $\mathrm{NaCl}$, a complex is formed changing the turbidity of the solution. The turbidity depends on the concentration of PEO. A spectrophotometer was used to measure the transmittance as a function of PEO concentration.

A calibration curve was obtained by preparing turbidimetric samples of PEO solutions of known concentration and measuring their transmittance. For PEO of molecular weight of 400,000, the calibration curve is shown in Figure 5.5. Similarly, the turbidimetric sample of the supernatant was prepared and its transmittance was measured and then from the calibration curve the concentration was determined. By subtracting the amount of PEO present in the supernatant from the amount of polymer initially added, the amount adsorbed by the particles was determined. 


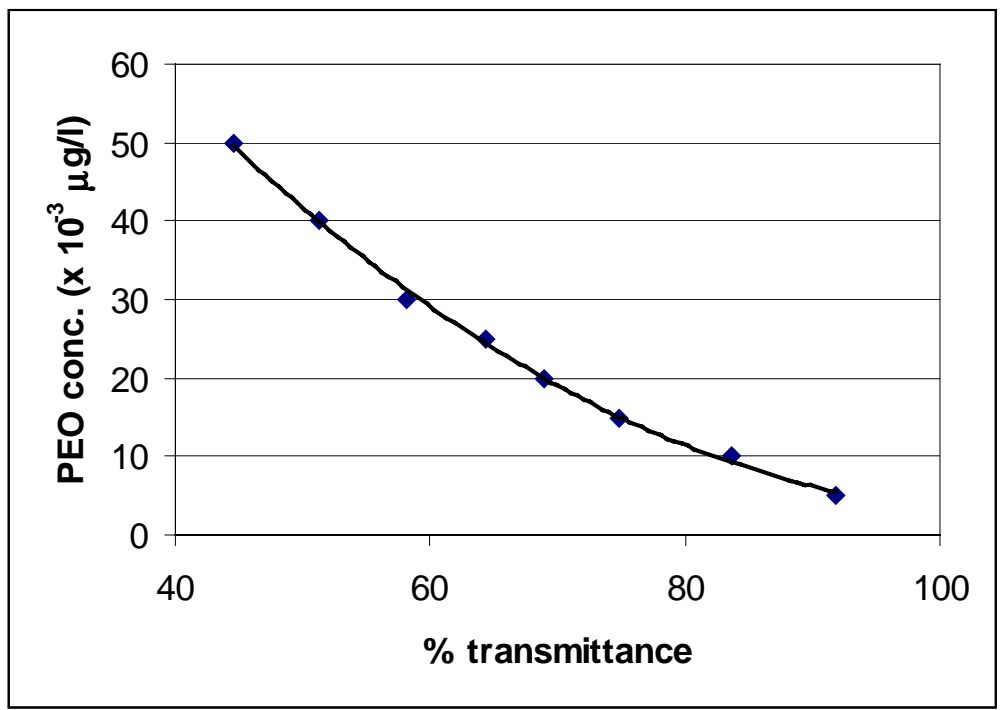

Figure 5.5. Turbidimetric calibration curve for 400,000 MW PEO in glycerin-water solution. 
It was found that as the concentration of the added PEO solution was increased, amount of PEO adsorbed, rather than reaching a plateau value, kept increasing. Beyond a certain concentration (>1400 ppm), PEO started to precipitate out. This behavior is expected because, as mentioned earlier, glycerin-water solution is a bad solvent for PEO. Therefore, it is safe to assume that at low concentration, all the PEO that is added for agglomeration gets adsorbed on the particle surface in the form of multiple layers.

\subsection{Estimation of Thickness of Adsorbed Polymer Layer}

In the absence of an experimental value of the amount of PEO needed for monolayer coverage, an estimation of this quantity is required. If the radius of a polymer molecule is $\mathrm{R}_{\mathrm{g}}$, then the number of molecules required to completely cover a smooth solid spherical particle would be:

$$
n=\frac{4 \pi b^{2}}{\pi R_{g}^{2}}
$$

If the total volume of particles is $\phi_{\mathrm{T}}$,

then the number of particles in the suspension $=\frac{\phi_{T}}{4 / 3 \pi b^{3}}$

Number of molecules required for monolayer coverage $=\frac{4 b^{2}}{R_{g}^{2}} \frac{3 \phi_{T}}{4 \pi b^{3}}=\frac{3 \phi_{T}}{\pi b R_{g}^{3}}$

Mass of polymer required for monolayer coverage $=\frac{3 \phi_{T} M_{w}}{\pi b R_{g}^{2} N_{A}}$

where $\mathrm{N}_{\mathrm{A}}$ is the Avagadro's number. If $\mathrm{P}_{0}$ is the mass of polymer added, then number of

$$
\text { monolayers }=\frac{P_{0}}{\frac{3 \phi_{T} M_{w}}{\pi b R_{g}^{2} N_{A}}}
$$


Furthermore, if it is assumed that the thickness of monolayer $\approx \mathrm{R}_{\mathrm{g}}$, then the total thickness of the polymer layer on a particle:

$$
\delta=\frac{\pi b R_{g}^{2} N_{A} P_{0}}{3 \phi_{T} M_{w}} R_{g}
$$

Volume of the polymer layer $\approx 4 \pi \mathrm{b} \delta[\delta+\mathrm{b}]$

polymer added per particle $=\frac{P_{0}}{3 \phi_{T} / 4 \pi b^{3}}$

Therefore, polymer concentration in the layer is obtained by dividing equation (5.17) by equation (5.16):

$$
c=\frac{b M_{w}}{\pi R_{g}^{3} N_{A}\left[\frac{\pi b R_{g}^{3} N_{A} P_{0}}{3 \phi_{T} M_{w}}+b\right]}
$$

Calculated values of $\mathrm{c}$ and $\delta$ are listed in Table (5.5).

\section{Table 5.5. Calculated values of $\mathrm{c}$ and $\delta$}

$$
\left(\mathrm{b}=2.46 \mu \mathrm{m}, \phi_{\mathrm{T}}=0.04 \mathrm{~cm}^{3}\right)
$$

\begin{tabular}{|c|c|c|c|c|c|c|}
\hline \multirow{2}{*}{$\begin{array}{c}\text { added } \\
\text { polymer }(\mathrm{gm})\end{array}$} & \multicolumn{2}{|c|}{$\mathbf{R}_{\mathrm{w}}=\mathbf{2 \times 1 0 ^ { 5 }}$} & \multicolumn{2}{c|}{$\mathbf{M}_{\mathrm{w}}=1 \times 10^{6}$} & \multicolumn{2}{c|}{$\mathbf{M}_{\mathrm{w}}=\mathbf{5 \times 1 0}$} \\
& $\mathbf{c}\left(\mathrm{gm} / \mathrm{cm}^{3}\right)$ & $\delta(\mathrm{nm})$ & $\mathbf{c}\left(\mathrm{gm} / \mathrm{cm}^{3}\right)$ & $\delta(\mathrm{nm})$ & $\mathbf{c}\left(\mathrm{gm} / \mathrm{cm}^{3}\right)$ & $\delta(\mathrm{nm})$ \\
\hline $4 \times 10^{-4}$ & 0.328 & 24.8 & 0.139 & 57.7 & 0.0587 & 132.6 \\
\hline $2 \times 10^{-3}$ & 0.316 & 123.6 & 0.127 & 288.5 & 0.0487 & 663.0 \\
\hline $4 \times 10^{-3}$ & 0.301 & 247.2 & 0.115 & 577.0 & 0.0402 & 1326 \\
\hline
\end{tabular}




\section{Chapter 6. Results and Discussion}

Agglomeration of particles in the suspension was carried out at different flocculation conditions. The number of agglomerates and their size were measured with respect to time using image analysis.

Figure (6.1) shows the digital image of a freshly prepared fully dispersed

suspension. Agglomerates formed after 4 minutes of shearing at $80 \mathrm{~s}^{-1}$ are shown in Figure (6.2) which shows that an initially well-dispersed particles now have clustered together to form agglomerates and there are fewer numbers of them. Image analysis was used to measure the size of these agglomerates and count their number at different time intervals. If the size of agglomerates is plotted with respect to time, agglomerate growth curves are obtained as given in Figure (6.3) which shows agglomeration at three different shear rates. As can be seen agglomerate size increases rapidly with time at first and then reaches a plateau. The initial growth period gives the collision efficiency and while the plateau region yields equilibrium or maximum floc size. Both these parameters are used to characterize the agglomeration process.

In the following sections, at first the collision efficiencies are determined from the agglomeration experiments and then the unknown terms in the collision efficiency model are obtained with the help of non-linear regression analysis. This is followed by a discussion of the effects of flocculation conditions on the collision efficiency. Then the results regarding the maximum floc size are presented and the effects of flocculation conditions on maximum floc size and agglomerate growth are discussed. 


\subsection{Experimental Determination of Collision Efficiency}

Recall that the rate of agglomeration is given by equation (2.28), which is reproduced here for convenience:

$\ln \frac{N}{N_{0}}=-\frac{4 \alpha \dot{\gamma} \phi}{\pi} t$

where, $\mathrm{N}_{0}$ is the number concentration at time $\mathrm{t}=0$ and $\mathrm{N}$ is the number concentration at any time t. If $\ln \left(\mathrm{N} / \mathrm{N}_{0}\right)$ is plotted with respect to time, equation (6.1) should give a straight line, and, from the slope of this line, $\alpha$ can be determined. However, this is applicable

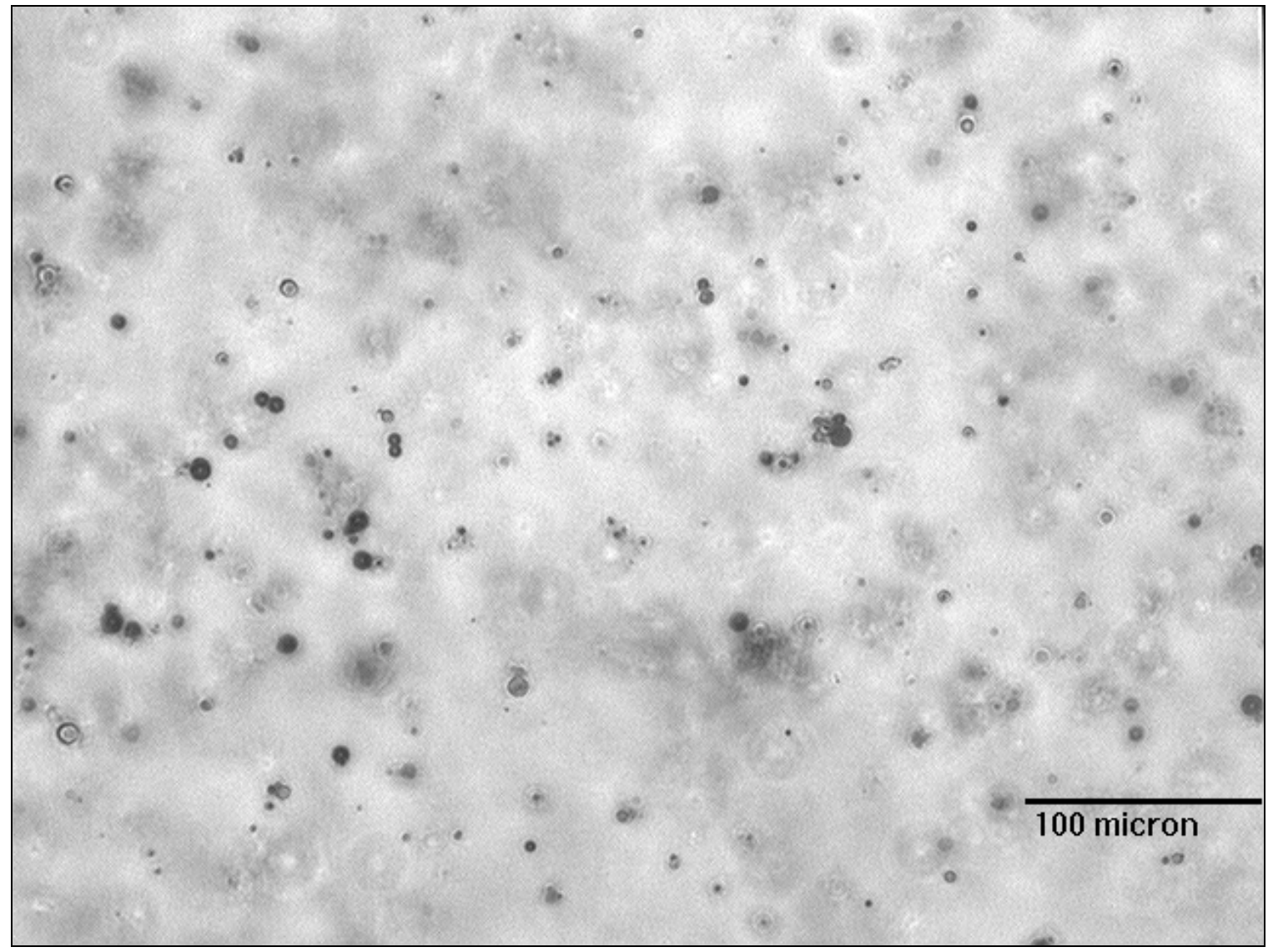

Figure 6.1. Image of particles in a freshly prepared suspension. Solid content $=0.2 \% \mathrm{v} / \mathrm{v}$. 


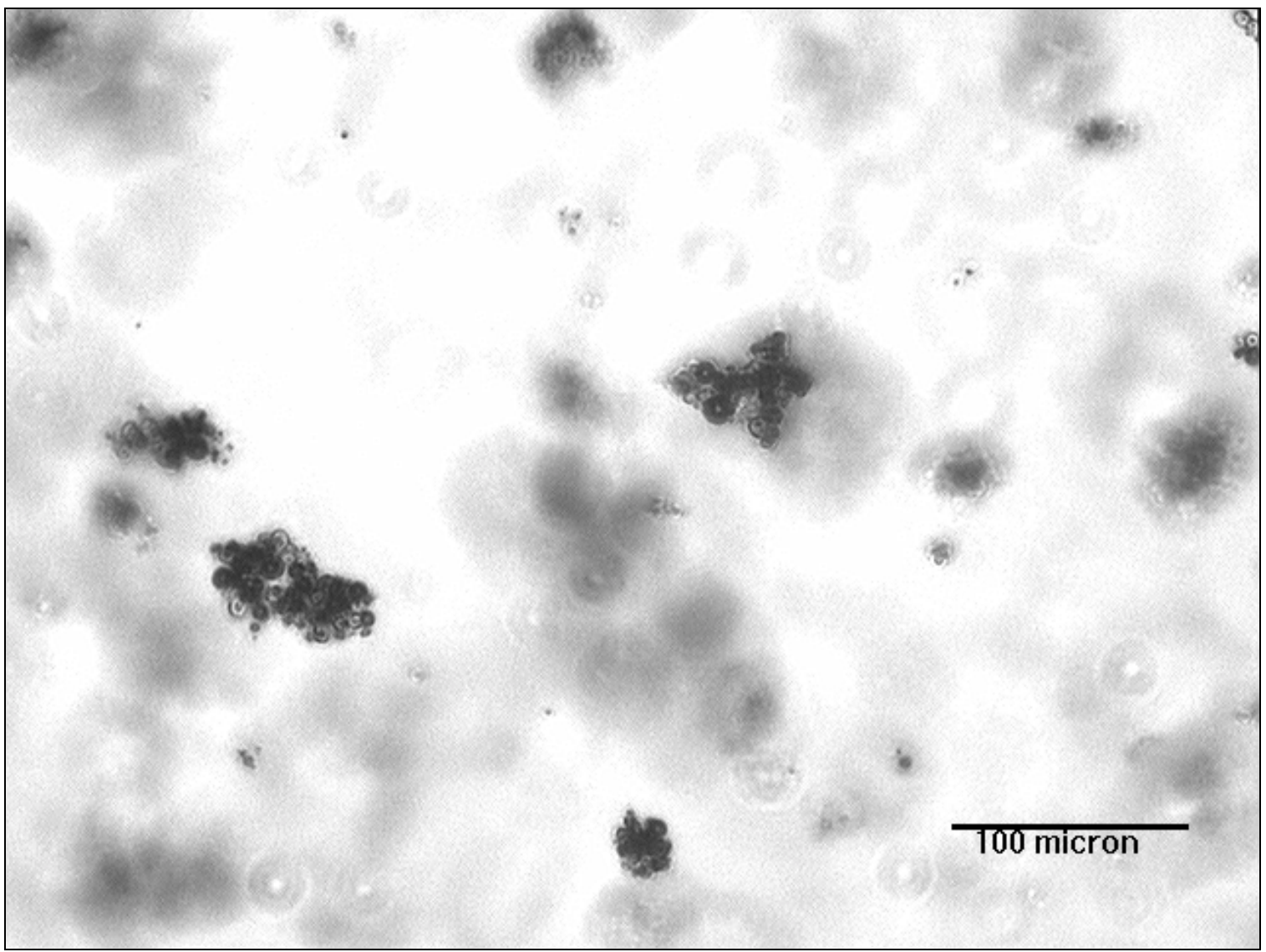

Figure 6.2. Image of agglomerates formed after 4 minutes of shearing at $80 \mathrm{~s}^{-1}$. Solid content $=0.2 \% \mathrm{v} / \mathrm{v}$, PEO conc. $=200 \mathrm{mg} / \mathrm{l}, \mathrm{MW}=1 \times 10^{6}$. 


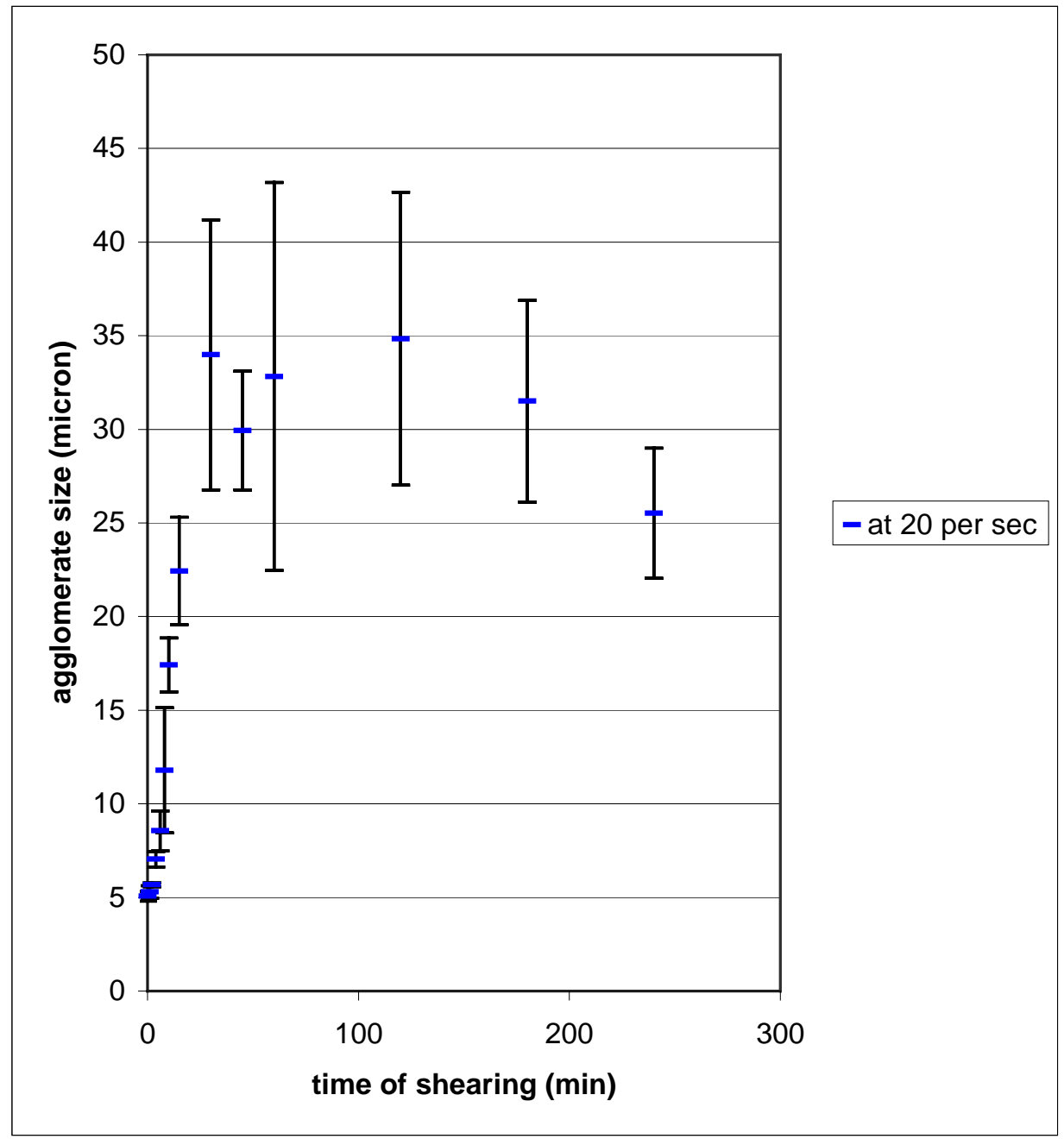

(a) 




(b) 




(c)

Figure 6.3. Agglomerate growth with respect to time at (a) $20 \mathrm{~s}^{-1}$, (b) $80 \mathrm{~s}^{-1}$ and (c) $190 \mathrm{~s}^{-1}$. Solid content $=0.2 \% \mathrm{v} / \mathrm{v}, \mathrm{PEO} \mathrm{MW}=1 \times 10^{6}, \mathrm{PEO}$ concentration $=100 \mathrm{mg} / \mathrm{l}$. 
only during the early stages of agglomeration when collisions are taking place chiefly between the primary particles.

The derivation of Smoluchowski's frequency and calculation of the collision efficiency are both meant for the situation where two primary particles are colliding to form a doublet. Therefore, to experimentally determine the collision efficiency, $\mathrm{N} / \mathrm{N}_{0}$ has to be measured with respect to time till it falls below 0.5 . This point indicates that most of the primary particles have formed a doublet, and, therefore the population of particles has reduced by half. By plotting $\mathrm{N} / \mathrm{N}_{0}$ vs. time, a straight-line can be fitted to the data and from which, time $\mathrm{t}_{1 / 2}$ for $\mathrm{N} / \mathrm{N}_{0}=0.5$ can be determined and the collision efficiency can be calculated from:

$\alpha_{\text {experimental }}=-\frac{\pi}{4 \dot{\gamma} \phi t_{1 / 2}} \ln (0.5)$

The calculation of collision efficiency from agglomeration experiments is illustrated with the help of an experiments performed at a shear rate $\dot{\gamma}$ of $80 \mathrm{~s}^{-1}$. The solid concentration was $\phi=0.2 \% \mathrm{v} / \mathrm{v}$ and PEO $\left(\mathrm{MW}=1 \times 10^{6}\right)$ concentration was $20 \mathrm{mg} / \mathrm{l}$. $\mathrm{N} / \mathrm{N}_{0}$ is plotted against time of shearing and this is shown in Figure (6.4). A straight line is easily fitted to the data. From the equation of the straight line $t_{1 / 2}$ is $470.64 \mathrm{~s}$ for $\mathrm{N} / \mathrm{N}_{0}=$ 0.5. From equation (6.2), the collision efficiency is calculated to be 0.0076 .

Plots of $\mathrm{N} / \mathrm{N}_{0}$ versus time for the early stages of agglomeration are given in Appendix (C) for all other experimental conditions. At each experimental condition, at least three repeat experiments were performed. In each plot, the best straight line and the corresponding regression coefficient $\left(\mathrm{R}^{2}\right)$ are also given. As can be seen from Appendix (C), good straight-line fits are obtained as evidenced by the high values of the regression coefficient $(\approx 0.9$ or better), which confirms the linear nature of the process during the 
early stage of agglomeration. During these initial stages samples were withdrawn at 1minute intervals and then at 2-minute intervals. In some cases a few minutes of shearing was necessary before the relative number concentration dropped by half. However, in other cases, the relative number concentration became less than half after just one minute of shearing, exhibiting relatively fast flocculation. In those cases, only two data points were available for fitting a straight-line. The calculated values of collision efficiency are provided in Table (6.1).

Table 6.1. Experimental values of collision efficiency

(a) $\phi=0.2 \%$, PEO MW $=1 \times 10^{6}$

\begin{tabular}{|c|c|c|c|c|c|c|c|c|c|}
\hline shear & \multicolumn{3}{|c|}{ PEO conc. $=20 \mathrm{mg} / \mathrm{l}$} & \multicolumn{3}{|c|}{$P E O$ conc. $=100 \mathrm{mg} / \mathrm{l}$} & \multicolumn{3}{|c|}{ PEO conc. $=200 \mathrm{mg} / \mathrm{l}$} \\
\hline $20 s^{-1}$ & 0.0257 & 0.0189 & 0.0189 & 0.0627 & 0.061 & 0.062 & 0.165 & 0.191 & 0.165 \\
\hline $80 \mathrm{~s}^{-1}$ & 0.0076 & 0.0076 & 0.0076 & 0.0176 & 0.020 & 0.015 & 0.082 & 0.078 & 0.078 \\
\hline $190 \mathrm{~s}^{-1}$ & 0.0036 & 0.0036 & 0.0036 & 0.013 & 0.011 & 0.0098 & 0.034 & 0.034 & 0.039 \\
\hline
\end{tabular}

(Highlighted values were used for non-linear regression analysis)

(b) ) $\phi=0.2 \%$, shear rate $=80 \mathrm{~s}^{-1}$, PEO conc. $=100 \mathrm{mg} / 1$

\begin{tabular}{|l|l|l|l|}
\hline $\mathbf{M W}=\mathbf{2} \times \mathbf{1 0}^{\mathbf{5}}$ & 0.0157 & 0.016 & 0.0149 \\
\hline $\mathbf{M W}=\mathbf{5} \times \mathbf{1 0}^{\mathbf{6}}$ & 0.0458 & 0.0481 & 0.0486 \\
\hline
\end{tabular}






Figure 6.4. Relative count of particles versus time of shearing at shear rate $=80 \mathrm{~s}^{-1}$. PEO concentration $20 \mathrm{mg} / \mathrm{l}$, solid content $=0.2 \% \mathrm{v} / \mathrm{v}, \mathrm{MW}=1 \times 10^{6}$. 


\subsection{Determination of Exponents Appearing in the Collision Efficiency Model}

The collision efficiency model as given by equation (4.27) contains constant $\mathrm{k}_{3}$ and exponents $\mathrm{x}_{3}, \mathrm{y}_{3}$ and $\mathrm{z}_{3}$ that are yet to be determined. Non-linear regression analysis together with experimentally determined values of the collision efficiency were used to determine these quantities. However, before non-linear regression could be applied to the data, a few modifications were made to the dimensionless groups to account for the fact that not all variables appearing in these groups were known. These modifications and calculations of dimensionless groups are discussed below.

The dimensionless group $\mathrm{C}_{\mathrm{A}}$, is given by equation (4.19). In order to calculate it, Hamaker constants for glass, the suspending medium (consisting of water and glycerol) and the polymer PEO are required. These are:

$$
\begin{aligned}
& A_{\text {water }}=3.7 \times 10^{-20} \mathrm{~J}[66] \\
& A_{\text {glycerin }}=6.6 \times 10^{-20} \mathrm{~J}[67] \\
& A_{\text {solvent }}=A_{0}=0.9 \times 6.6 \times 10^{-20}+0.1 \times 3.7 \times 10^{-20}=6.31 \times 10^{-20} \mathrm{~J} \\
& A_{\text {glass }}=A_{1}=1.343 \times 10^{-20} \mathrm{~J}[66]
\end{aligned}
$$

The Hamaker constant for PEO could not be found in the literature and therefore an approximation was required. The Hamaker constants for other polymers such as polymethycrylate, polyvinyl chloride, polystyrene, polyethylene, polypropylene, polyisobutylene and polytetrafluoroethylene all fall in the range of $4-6.3 \times 10^{-20} \mathrm{~J}[66$, 68]. The Hamaker constant of polyethylene is $6.3 \times 10^{-20} \mathrm{~J}$ [68]. Assuming that the Hamaker constant for PEO would be similar to that of polyethylene, the value was taken to be $\mathrm{A}_{2} \approx 6 \times 10^{-20} \mathrm{~J}$. 
The effective Hamaker constant as appearing in equations (4.19) and (4.23) then becomes:

$\left(\mathrm{A}_{0}{ }^{1 / 2}-\mathrm{A}_{2}{ }^{1 / 2}\right)\left(\mathrm{A}_{2}{ }^{1 / 2}-\mathrm{A}_{1}^{1 / 2}\right) \approx 10^{-22} \mathrm{~J}$.

Since only one average size of particles was used and the suspension medium remains the same, putting $\mathrm{b}=2.47 \times 10^{-6} \mathrm{~m}$ and $\eta=0.19 \mathrm{~Pa}$-s gives the dimensionless group $\mathrm{C}_{\mathrm{A}}$ " as:

$C_{A^{\prime \prime}}=\frac{1.892 \times 10^{-18}\left(2.46 \times 10^{-6}+\delta\right)}{\dot{\gamma} \delta^{2}}$

The above shows that for the given system $\mathrm{C}_{\mathrm{A}}$, depends on the adsorbed polymer layer thickness and the shear rate. For the conditions used in this work, calculated values of $\mathrm{C}_{\mathrm{A}}$, are given in Table (6.2).

Next the dimensionless group, $\mathrm{C}_{\mathrm{R}}$, given by equation (4.23) is calculated. For this, the surface potential of particles $\psi_{0}$ and Debye-Huckle parameter $\kappa$ are required. Both these quantities are not known. They depend on $\mathrm{pH}$ and electrolyte concentration of the suspension. Since $\mathrm{pH}$ and electrolyte concentration remain constant in this work, both $\psi_{0}$ and $\kappa$ can be assumed to be constant for the conditions used in this work. The remaining parameter is given as:

$\mathrm{C}_{\mathrm{R}},=2.759 \times 10^{13} \delta$

where electrical permittivity of glycerin-water solution $\varepsilon_{\mathrm{r}} \varepsilon_{0}=4.391 \times 10^{-10}$ coulomb $^{2} / \mathrm{N}-\mathrm{m}^{2}$ [69]. Calculated values of $C_{R}$, are given in Table (6.3).

For the experimental conditions, the steric interaction dimensionless parameter is given by equation (4.26). Here $\mathrm{k}_{\mathrm{B}}=1.38 \times 10^{-23} \mathrm{~J} / \mathrm{K}, \mathrm{T}=298 \mathrm{~K}, \mathrm{~V}_{1}=9.273 \times 10^{-29}$ $\mathrm{m}^{3} /$ molecule, $\rho_{2}=1.13 \times 10^{3} \mathrm{~kg} / \mathrm{m}^{3}, \eta=0.19$ Pa-s and $\chi=0.50036$, which results in: 
Table 6.2. Calculated values of $\mathrm{C}_{\mathrm{A}}$ "

\begin{tabular}{|c|c|c|c|c|}
\hline \multicolumn{5}{|c|}{ Molecular Weight $=\mathbf{2 0 0 , 0 0 0}$} \\
\hline $\begin{array}{c}\text { Polymer } \\
\text { added (gm) }\end{array}$ & $\delta(\mathbf{m})$ & $\dot{\gamma}=20 \mathrm{~s}^{-1}$ & $\dot{\gamma}=80 \mathrm{~s}^{-1}$ & $\dot{\gamma}=190 \mathrm{~s}^{-1}$ \\
\hline $4 \times 10^{-4}$ & $2.478 \times 10^{-8}$ & $3.828 \times 10^{-10}$ & $9.570 \times 10^{-11}$ & $4.029 \times 10^{-11}$ \\
\hline $2 \times 10^{-3}$ & $1.236 \times 10^{-7}$ & $1.599 \times 10^{-11}$ & $3.999 \times 10^{-12}$ & $1.684 \times 10^{-12}$ \\
\hline $4 \times 10^{-3}$ & $2.472 \times 10^{-7}$ & $4.191 \times 10^{-12}$ & $1.047 \times 10^{-12}$ & $4.411 \times 10^{-13}$ \\
\hline \multicolumn{5}{|c|}{ Molecular Weight $=1,000,000$} \\
\hline $\begin{array}{c}\text { Polymer } \\
\text { added (gm) }\end{array}$ & $\delta(\mathbf{m})$ & $\dot{\gamma}=20 \mathrm{~s}^{-1}$ & $\dot{\gamma}=80 \mathrm{~s}^{-1}$ & $\dot{\gamma}=190 \mathrm{~s}^{-1}$ \\
\hline $4 \times 10^{-4}$ & $5.77 \times 10^{-8}$ & $7.154 \times 10^{-11}$ & $1.788 \times 10^{-11}$ & $7.530 \times 10^{-12}$ \\
\hline $2 \times 10^{-3}$ & $2.885 \times 10^{-7}$ & $3.124 \times 10^{-12}$ & $7.809 \times 10^{-13}$ & $3.288 \times 10^{-13}$ \\
\hline $4 \times 10^{-3}$ & $5.77 \times 10^{-7}$ & $8.629 \times 10^{-13}$ & $2.157 \times 10^{-13}$ & $9.084 \times 10^{-14}$ \\
\hline \multicolumn{5}{|c|}{ Molecular Weight $=5,000,000$} \\
\hline $\begin{array}{c}\text { Polymer } \\
\text { added (gm) }\end{array}$ & $\delta(\mathrm{m})$ & $\dot{\gamma}=20 \mathrm{~s}^{-1}$ & $\dot{\gamma}=80 \mathrm{~s}^{-1}$ & $\dot{\gamma}=190 \mathrm{~s}^{-1}$ \\
\hline $4 \times 10^{-4}$ & $1.326 \times 10^{-7}$ & $1.395 \times 10^{-11}$ & $3.487 \times 10^{-12}$ & $1.468 \times 10^{-12}$ \\
\hline $2 \times 10^{-3}$ & $6.63 \times 10^{-7}$ & $6.721 \times 10^{-13}$ & $1.680 \times 10^{-13}$ & $7.075 \times 10^{-14}$ \\
\hline $4 \times 10^{-3}$ & $1.326 \times 10^{-6}$ & $2.037 \times 10^{-13}$ & $5.092 \times 10^{-14}$ & $2.144 \times 10^{-14}$ \\
\hline
\end{tabular}


Table 6.3. Calculated values of $C_{R}$,

\begin{tabular}{|l|l|l|}
\hline Polymer added (gm) & $\boldsymbol{\delta}(\mathbf{m})$ & Molecular Weight $=\mathbf{2 0 0 , 0 0 0}$ \\
\hline $4 \times 10^{-4}$ & $2.478 \times 10^{-8}$ & $6.837 \times 10^{5}$ \\
\hline $2 \times 10^{-3}$ & $1.236 \times 10^{-7}$ & $3.410 \times 10^{6}$ \\
\hline $4 \times 10^{-3}$ & $2.472 \times 10^{-7}$ & $6.820 \times 10^{6}$ \\
\hline Polymer added (gm) & $\boldsymbol{\delta}(\mathbf{m})$ & Molecular Weight $=\mathbf{1 , 0 0 0 , 0 0 0}$ \\
\hline $4 \times 10^{-4}$ & $5.770 \times 10^{-8}$ & $1.592 \times 10^{6}$ \\
\hline $2 \times 10^{-3}$ & $2.885 \times 10^{-7}$ & $7.959 \times 10^{6}$ \\
\hline $4 \times 10^{-3}$ & $5.770 \times 10^{-7}$ & $1.592 \times 10^{7}$ \\
\hline Polymer added (gm) & $\boldsymbol{\delta}(\mathbf{m})$ & Molecular Weight $=\mathbf{5 , 0 0 0 , 0 0 0}$ \\
\hline $4 \times 10^{-4}$ & $1.326 \times 10^{-7}$ & $3.658 \times 10^{6}$ \\
\hline $2 \times 10^{-3}$ & $6.63 \times 10^{-7}$ & $1.829 \times 10^{7}$ \\
\hline $4 \times 10^{-3}$ & $1.326 \times 10^{-6}$ & $3.658 \times 10^{7}$ \\
\hline
\end{tabular}


$C_{S^{\prime}}=7.249 \times 10^{9} \frac{c^{2} \delta^{2}}{\dot{\gamma}}$

Calculated values of $\mathrm{C}_{\mathrm{S}}$, are given in Table (6.4).

Finally, the collision efficiency according to the proposed model becomes:

$$
\alpha=k^{\prime}\left(\frac{1.892 \times 10^{-18}\left(2.46 \times 10^{-6}+\delta\right)}{\dot{\gamma} \delta^{2}}\right)^{x_{3}}\left(2.759 \times 10^{13} \delta\right)^{y_{3}}\left(7.249 \times 10^{9} \frac{c^{2} \delta^{2}}{\dot{\gamma}}\right)^{z_{3}}
$$

To perform the non-linear regression analysis, Polymath 5.0 numerical analysis software was used. The dependent variable was the experimentally determined value of collision efficiency and the independent variables were the corresponding dimensionless numbers $C_{A}, C_{R}$, and $C_{S}$. Data used for non-linear regression are given in Table (6.5). To carry out the regression analysis, initial guesses of $\mathrm{k}^{\prime}, \mathrm{x}_{3}, \mathrm{y}_{3}$ and $\mathrm{z}_{3}$ are required. Various combinations of initial guesses were used. For some combinations, regression did not converge and no solutions were obtained. However, for some combinations of initial guesses regression converged and values of parameters were obtained. The software also gives the $95 \%$ confidence interval for the parameter values and the coefficient of regression for each successful regression analysis. Therefore, these two criteria were employed to select the best values of the parameters. For the best values of regression analysis, the $95 \%$ confidence interval should be small while the coefficient of regression should be high. Also, the values should be physically realistic. Following the above-laid criteria, the values of $\mathrm{k}^{\prime}, \mathrm{x}_{3}, \mathrm{y}_{3}$ and $\mathrm{z}_{3}$ were obtained and they are given in Table (6.6) together with their 95\% CI. With these values the coefficient of regression was 0.978 , signifying a good fit to the data. For the range of experimental conditions studied in this work, the collision efficiency can be predicted from:

$$
\alpha=1.472 \times 10^{-6}\left(C_{A^{\prime \prime}}\right)^{0.185}\left(C_{R^{\prime}}\right)^{1.000}\left(C_{S}\right)^{0.432}
$$


Table 6.4. Calculated values of $\mathrm{C}_{S}$

\begin{tabular}{|c|c|c|c|c|c|}
\hline \multicolumn{6}{|c|}{ Molecular Weight $=200,000$} \\
\hline $\begin{array}{l}\text { Polymer } \\
\text { added (gm) }\end{array}$ & $\overline{c\left(k g / \mathrm{m}^{3}\right)}$ & $\delta(\mathbf{m})$ & $\dot{\gamma}=20 \mathrm{~s}^{-1}$ & $\dot{\gamma}=80 \mathrm{~s}^{-1}$ & $\dot{\gamma}=190 \mathrm{~s}^{-1}$ \\
\hline $4 \times 10^{-4}$ & $0.328 \times 10^{3}$ & $2.478 \times 10^{-8}$ & $2.394 \times 10^{-2}$ & $5.986 \times 10^{-3}$ & $2.52 \times 10^{-3}$ \\
\hline $2 \times 10^{-3}$ & $0.316 \times 10^{3}$ & $1.236 \times 10^{-7}$ & 0.5529 & 0.1382 & $5.82 \times 10^{-2}$ \\
\hline $4 \times 10^{-3}$ & $0.301 \times 10^{3}$ & $2.472 \times 10^{-7}$ & 2.007 & 0.5017 & 0.2112 \\
\hline \multicolumn{6}{|c|}{ Molecular Weight $=1,000,000$} \\
\hline $\begin{array}{l}\text { Polymer } \\
\text { added (gm) }\end{array}$ & $c\left(\mathrm{~kg} / \mathrm{m}^{3}\right)$ & $\delta(\mathbf{m})$ & $\dot{\gamma}=20 \mathrm{~s}^{-1}$ & $\dot{\gamma}=80 \mathrm{~s}^{-1}$ & $\dot{\gamma}=190 \mathrm{~s}^{-1}$ \\
\hline $4 \times 10^{-4}$ & $0.139 \times 10^{3}$ & $5.770 \times 10^{-8}$ & $2.331 \times 10^{-2}$ & $5.829 \times 10^{-3}$ & $2.45 \times 10^{-3}$ \\
\hline $2 \times 10^{-3}$ & $0.127 \times 10^{3}$ & $2.885 \times 10^{-7}$ & 0.4866 & 0.1216 & $5.13 \times 10^{-2}$ \\
\hline $4 \times 10^{-3}$ & $0.115 \times 10^{3}$ & $5.770 \times 10^{-7}$ & 1.596 & 0.399 & 0.168 \\
\hline \multicolumn{6}{|c|}{ Molecular Weight $=5,000,000$} \\
\hline $\begin{array}{l}\text { Polymer } \\
\text { added (gm) }\end{array}$ & $c\left(\mathrm{~kg} / \mathrm{m}^{3}\right)$ & $\delta(\mathbf{m})$ & $\dot{\gamma}=20 \mathrm{~s}^{-1}$ & $\dot{\gamma}=80 \mathrm{~s}^{-1}$ & $\dot{\gamma}=190 \mathrm{~s}^{-1}$ \\
\hline $4 \times 10^{-4}$ & $0.059 \times 10^{3}$ & $1.326 \times 10^{-7}$ & $2.196 \times 10^{-2}$ & $5.489 \times 10^{-3}$ & $2.311 \times 10^{-3}$ \\
\hline $2 \times 10^{-3}$ & $0.049 \times 10^{3}$ & $6.63 \times 10^{-7}$ & 0.378 & $9.446 \times 10^{-2}$ & $3.978 \times 10^{-2}$ \\
\hline $4 \times 10^{-3}$ & $0.040 \times 10^{3}$ & $1.326 \times 10^{-6}$ & 1.029 & 0.257 & 0.108 \\
\hline
\end{tabular}


Table 6.5. Data used for non-linear regression

\begin{tabular}{|l|l|l|l|l|l|}
\hline \multicolumn{1}{|c|}{$\begin{array}{c}\text { Shear } \\
\text { Rate }\end{array}$} & \multicolumn{1}{|c|}{$\begin{array}{c}\text { Polymer Conc. } \\
\left(\mathbf{s}^{-1}\right)\end{array}$} & $\begin{array}{c}\text { Experimental } \\
\text { Collision } \\
\text { Efficiency }\end{array}$ & \multicolumn{1}{|c|}{$\mathbf{C}_{\mathbf{A}}}$, & $\mathbf{C}_{\mathbf{R}}{ }^{\prime}$ & $\mathbf{C}_{\mathbf{S}}$ \\
\hline 20 & 100 & 0.0627 & $3.124 \times 10^{-12}$ & $7.959 \times 10^{6}$ & 0.4866 \\
\hline 80 & 100 & 0.0176 & $7.809 \times 10^{-13}$ & $7.959 \times 10^{6}$ & 0.1216 \\
\hline 190 & 100 & 0.013 & $3.288 \times 10^{-13}$ & $7.959 \times 10^{6}$ & 0.05128 \\
\hline 20 & 200 & 0.165 & $8.629 \times 10^{-13}$ & $1.592 \times 10^{7}$ & 1.596 \\
\hline 80 & 200 & 0.082 & $2.157 \times 10^{-13}$ & $1.592 \times 10^{7}$ & 0.399 \\
\hline 190 & 200 & 0.034 & $9.084 \times 10^{-14}$ & $1.592 \times 10^{7}$ & 0.168 \\
\hline 20 & 20 & 0.0189 & $7.154 \times 10^{-11}$ & $1.592 \times 10^{6}$ & 0.0233 \\
\hline 80 & 20 & 0.0076 & $1.788 \times 10^{-11}$ & $1.592 \times 10^{6}$ & $5.829 \times 10^{-3}$ \\
\hline 190 & 20 & 0.0036 & $7.530 \times 10^{-12}$ & $1.592 \times 10^{6}$ & $2.454 \times 10^{-3}$ \\
\hline
\end{tabular}


To further validate the model, the collision efficiencies were predicted for conditions which were not used as the model inputs. The comparison is given in Table (6.7) and it can be seen that the predicted values are in agreement with the experimental values, thus this model can be successfully used for prediction. This model should be applicable for the agglomeration conditions for which the dimensionless parameters fall within the range as given in Table(6.5). It should be noted that this model is not applicable for suspensions where no polymer flocculant is present.

From this model, it should be possible to assess the effects of agglomeration conditions on the collision efficiency where surface of the particles is completely covered with polymer. These effects are discussed in the following sections.

Table 6.6. Results of Regression Analysis

\begin{tabular}{|l|l|l|}
\hline Parameter & Value & $95 \%$ CI \\
\hline $\mathrm{k}^{\prime}$ & $1.472 \times 10^{-6}$ & $4.801 \times 10^{-7}$ \\
\hline $\mathrm{x}_{3}$ & 0.185 & 0.012 \\
\hline $\mathrm{y}_{3}$ & 1.000 & 0.019 \\
\hline $\mathrm{z}_{3}$ & 0.432 & 0.153 \\
\hline
\end{tabular}


Table 6.7. Comparison of Predicted and Experimental Values of Collision Efficiency $(\phi=0.2 \% \mathrm{v} / \mathrm{v}$, PEO concentration $=100 \mathrm{mg} / \mathrm{l})$

\begin{tabular}{|c|c|c|c|c|c|c|}
\hline $\begin{array}{c}\text { Shear rate } \\
\left(\mathbf{s}^{-\mathbf{1}}\right)\end{array}$ & $\mathbf{M W}$ & $\mathbf{C}_{\mathbf{A}^{\prime}}$ & $\mathbf{C}_{\mathbf{R}^{\prime}}$ & $\mathbf{C}_{\mathbf{S}}$ & $\boldsymbol{\alpha}_{\text {pred }}$ & $\boldsymbol{\alpha}_{\text {exp }}$ \\
\hline 80 & $2 \times 10^{5}$ & $3.99 \times 10^{-12}$ & $3.41 \times 10^{6}$ & $5.82 \times 10^{-2}$ & 0.0114 & 0.012 \\
\hline 130 & $1 \times 10^{6}$ & $4.81 \times 10^{-13}$ & $7.96 \times 10^{6}$ & $7.48 \times 10^{-2}$ & 0.0201 & 0.021 \\
\hline 80 & $5 \times 10^{6}$ & $1.68 \times 10^{-13}$ & $1.83 \times 10^{7}$ & $9.45 \times 10^{-2}$ & 0.048 & 0.042 \\
\hline
\end{tabular}

\subsection{Effect of Polymer Concentration on Collision Efficiency}

To study the effect of amount of flocculant added to the suspension, experiments were performed at three levels of PEO concentration- 20, 100 and $200 \mathrm{mg} / \mathrm{l}$. The Molecular weight of PEO was $1 \times 10^{6}$ and the glass bead concentration was $0.2 \% \mathrm{v} / \mathrm{v}$. Figure (6.5) shows the effect of PEO concentration on the collision efficiency at three shear rates $-20,80$ and $190 \mathrm{~s}^{-1}$. Each point is an average of three experiments and error bars display the spread of \pm one standard deviation. As can be seen, the collision efficiency increases with increasing concentration of PEO. This occurs because the thickness of the polymer layer increases with PEO concentration as shown by equation (5.15) and corresponding values in Table (5.5). This means that polymer segments protrude out in the suspending medium to a greater length and this increases the effective diameter of the solid particles. If all the terms involving the polymer layer thickness in the collision efficiency of equation (6.7), are combined, it is found that the collision efficiency is proportional to $\delta^{1.494}$. 




Figure 6.5. Effect of polymer concentration on the collision efficiency. The suspension contained $0.2 \% \mathrm{v} / \mathrm{v}$ solid particles and MW of PEO was $1 \times 10^{6}$. 
Due to the presence of polymer segments extending into the solution, the solid particles that approach each other begin to interact with each other at a greater interparticle distance than in the case with bare particles. This is also reflected in the increasing magnitudes of the electric repulsion parameter $C_{R}$, and the steric interaction parameter $C_{S}$ as given in Table (6.3) and Table (6.4) respectively, with increasing polymer concentration. Since in this case the steric interaction energy is attractive, a thicker polymer layer provides stronger binding between the particles, thus, promoting agglomeration. Note that in order for the polymer segments to able to form a bridge between the particles, it is necessary that the polymer segments extend beyond the electric double-layer thickness $\left(\kappa^{-1}\right)$. If they do not, electric double-layer will overlap first and repel the particles before the polymer layer can interact and let steric attraction pulls the particles together as they approach each other.

At this stage it would be relevant to point out that a higher collision efficiency with increasing polymer layer thickness is observed in this work because the steric interaction is attractive due to an unfavorable interaction between PEO and the aqueous glycerin solution as discussed earlier. If conditions were different and the suspension had been prepared in a solution which was a good solvent for PEO, polymer segments would have repelled each other preventing agglomeration and this would be termed steric stabilization. In such a case, exponent of steric interaction term $x_{3}$, would be negative signifying that any change in agglomeration conditions that increases $\mathrm{C}_{\mathrm{S}}$ would reduce the collision efficiency. 


\subsection{Effect of Shear Rate on Collision Efficiency}

Experiments were performed to study the effect of shear rate on the collision efficiency. This was an important aspect of the work because very few studies are available where the effect of shear rate on the collision efficiency had been examined in case of polymer bridging. The effect of shear rate on orthokinetic coagulation is of course, well studied even theoretically as discussed in section (2.5). In the absence of electric double-layer repulsion, the collision efficiency varies as -0.18 power of shear rate (see equation 2.28).

To find out how the collision efficiency varies with shear rate in case of polymer bridging, agglomeration experiments were performed at three levels of shear rate- 20, 80 and $190 \mathrm{~s}^{-1}$ and three levels of PEO (MW = $1 \times 10^{6}$ ) concentration of 20, 100 and 200 $\mathrm{mg} / \mathrm{l}$. Three repeat experiments were carried out at each combination of experimental conditions. Dependence of collision efficiency on shear rate is shown in Figure (6.6). Here the error bars represent a spread of \pm one standard deviation. A power law equation was fitted to the data to obtain the shear rate dependence.

As can be seen, the collision efficiency decreases with increasing shear rate. However, the rate at which it decreases depends on the concentration of PEO. At high PEO concentration, the collision efficiency is less dependent on the shear rate than at low PEO concentration though the difference is very small. This observation is similar to one made by Aunins [29], who also found that with increasing concentration of polymer flocculant, the collision efficiency becomes less dependent on the shear rate. 


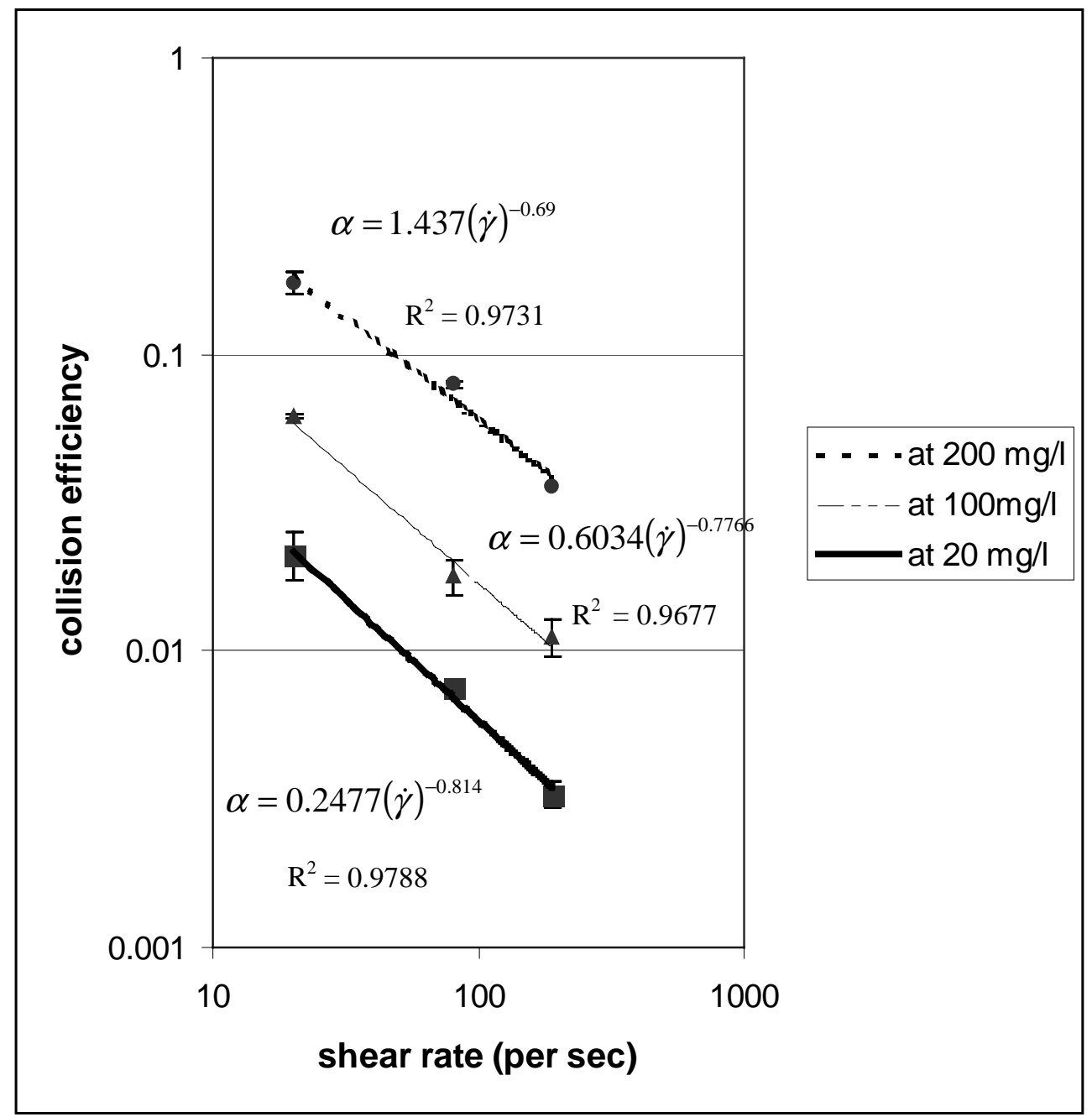

Figure 6.6. Effect of shear rate on the collision efficiency at different PEO concentrations. PEO MW $=1 \times 10^{6}$ and solid content $=0.2 \% \mathrm{v} / \mathrm{v}$. 
As the concentration of PEO is lowered from $200 \mathrm{mg} / \mathrm{l}$ to $20 \mathrm{mg} / \mathrm{l}$ there is still enough polymer to cover the entire surface of the particles. Therefore the change in shear rate dependence from high concentration to low concentration is not very significant. However if polymer were present in such a quantity that only partial surface is covered, there would be more significant change in shear rate dependence. The model presented in equation (4.9) can be used to explain this behavior. As mentioned earlier, if the polymer concentration in suspension is increased progressively, the mechanism of agglomeration changes from coagulation dominated to polymer bridging dominated as $\theta$ goes from 0 to 1. Correspondingly, the collision efficiency will have contributions from equations (4.6), (4.7) and (4.8) and each of which will contribute a power law shear rate dependence as given by $x_{1}, x_{2}$, and $x_{3}+z_{3}$ respectively. If it is assumed that in case of bare-bare surface interaction, the collision efficiency's shear rate dependence would be equal to as was obtained from trajectory analysis, $\mathrm{x}_{1}$ would be -0.18 (equation 2.28 ). On the other hand, in the case of covered-covered surface interaction shear rate dependence can be given by the values obtained in this work. The shear rate dependence for covered-bare surface interaction is not known. But the net shear rate dependence of the collision efficiency would have contributions from all three interactions each with their own shear rate dependence. The relative contribution will depend on the concentration and hence the shear rate dependence of the collision efficiency will change with the concentration of flocculant. 


\subsection{Effect of Molecular Weight of PEO on Collision Efficiency}

PEO of molecular weights of $2 \times 10^{5}, 1 \times 10^{6}$ and $5 \times 10^{6}$ was used to determine the effect of molecular weight on the collision efficiency. The concentration of PEO was kept constant at 100mg/l and agglomeration experiments were performed at a fixed shear rate of $80 \mathrm{~s}^{-1}$. Figure (6.7) shows the effect of molecular weight on the collision efficiency. The collision efficiency increases with increasing molecular weight. This is expected because high MW polymer would have thicker layer of adsorbed polymer owing to the larger size of the polymer molecule, thus making it easier for particles to come in contact with each other and form an agglomerate.

Gregory [21] also observed increased rate of agglomeration with increasing molecular weight of a cationic copolymer of acrylamide and dimethylamino-ethylacrylate which was used to flocculate silica particles (1-3 $\mu \mathrm{m}$ size) in a batch stirrer. Though no collision efficiency determinations were made but an increased rate of agglomeration suggests that high collision efficiency is obtained for high molecular weight polymer.

\subsection{Effect of Shear Rate on Agglomerate Size}

The shear rate at which agglomeration takes place has a significant effect on the growth of agglomerates and their maximum size. Experiments were performed at three different shear rates to observe the agglomerate growth with respect to time. Agglomerate size was measured as the number average agglomerate diameter at any given time. 


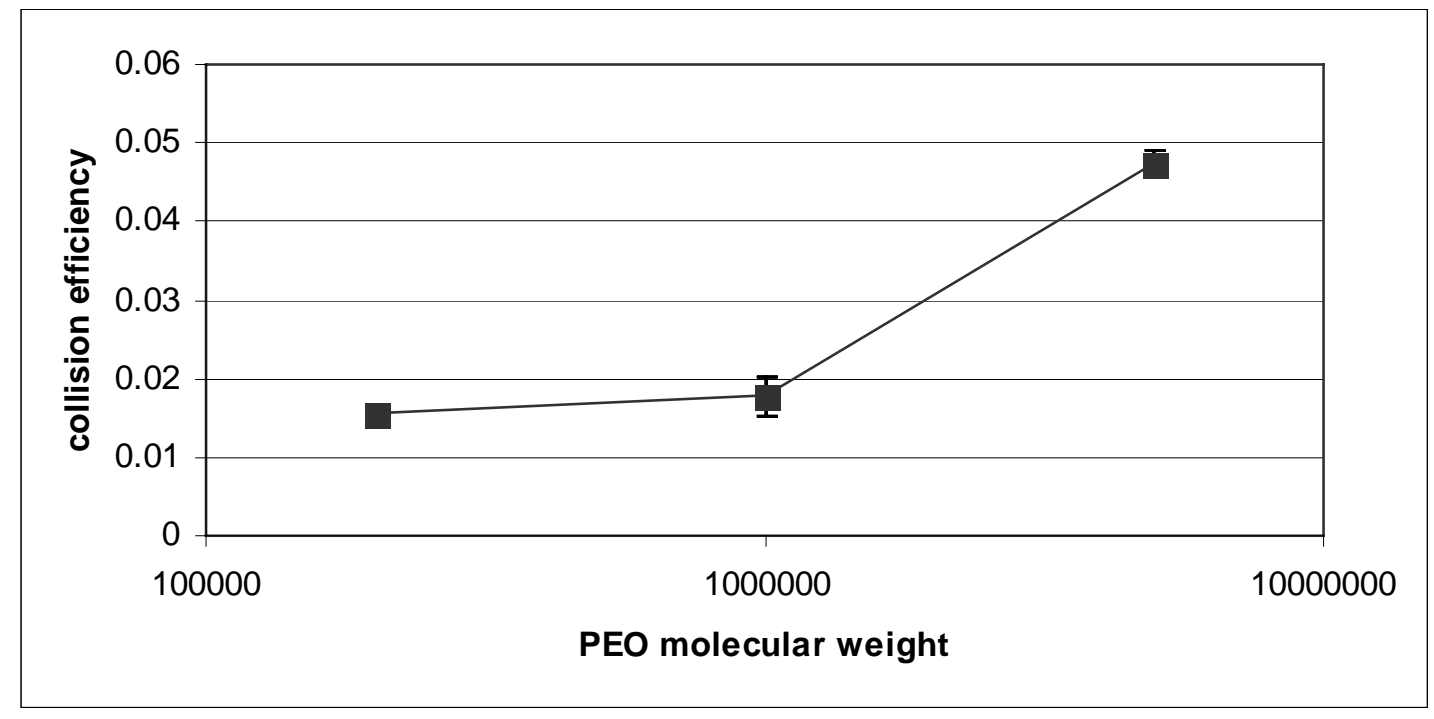

Figure 6.7. Effect of MW of PEO on the collision efficiency (shear rate $=80 \mathrm{~s}^{-1}$, PEO conc. $=100 \mathrm{mg} / \mathrm{l}$, solid content $=0.2 \% \mathrm{v} / \mathrm{v})$. 
Molecular weight and concentration of PEO were kept constant. Figure (6.8) shows the images of agglomerates at equilibrium at three shear rates. It can be seen that agglomerates produced at $20 \mathrm{~s}^{-1}$ are much bigger than that produced at $190 \mathrm{~s}^{-1}$. Maximum agglomerate size as a function of shear rate is plotted in Figure (6.9). Here, maximum agglomerate size $d_{f, 95 \%}$ is defined as the agglomerate size for which $95 \%$ of particles are smaller than it. The power law dependence is given by:

$d_{f, 95 \%}=199.88(\dot{\gamma})^{-0.258}$

Here, $d_{f, 95 \%}$ is in $\mu \mathrm{m}$ and shear rate is in $\mathrm{s}^{-1}$. As can be seen, small agglomerates are produced at high shear rate. This occurs because at high shear rates, an agglomerate experiences a high shear stress and hence only smaller agglomerates survive as discussed in section 2.7. This kind of shear rate dependence has been used widely to express the maximum floc size as a function of shear rate both in laminar and turbulent flow fields. Smith and Kitchener [50] performed floc breakage experiments in laminar flow field using a Couette device with glass beads in the range of 5-40 $\mu \mathrm{m}$ size which were agglomerated with a commercial flocculant. The floc size was found to be proportional to -0.2 power of shear rate between $50-200 \mathrm{~s}^{-1}$. For polystyrene latex particles of $2.17 \mu \mathrm{m}$ coagulated with $\mathrm{NaCl}$ in a Couette device, the equilibrium agglomerate size found to be dependent not only on shear rate but also on the solid volume fraction [34]. For volume fractions of $1 \times 10^{-5}$ and $5 \times 10^{-5}$ shear rate dependence changed from -0.2651 to -0.1863 . Floc breakage studies performed with other systems where particle size, flocculant, dose and mixing devices varied show that the exponent ranges from -0.3 to -1.5 [70], which suggest that it depends on many factors. 


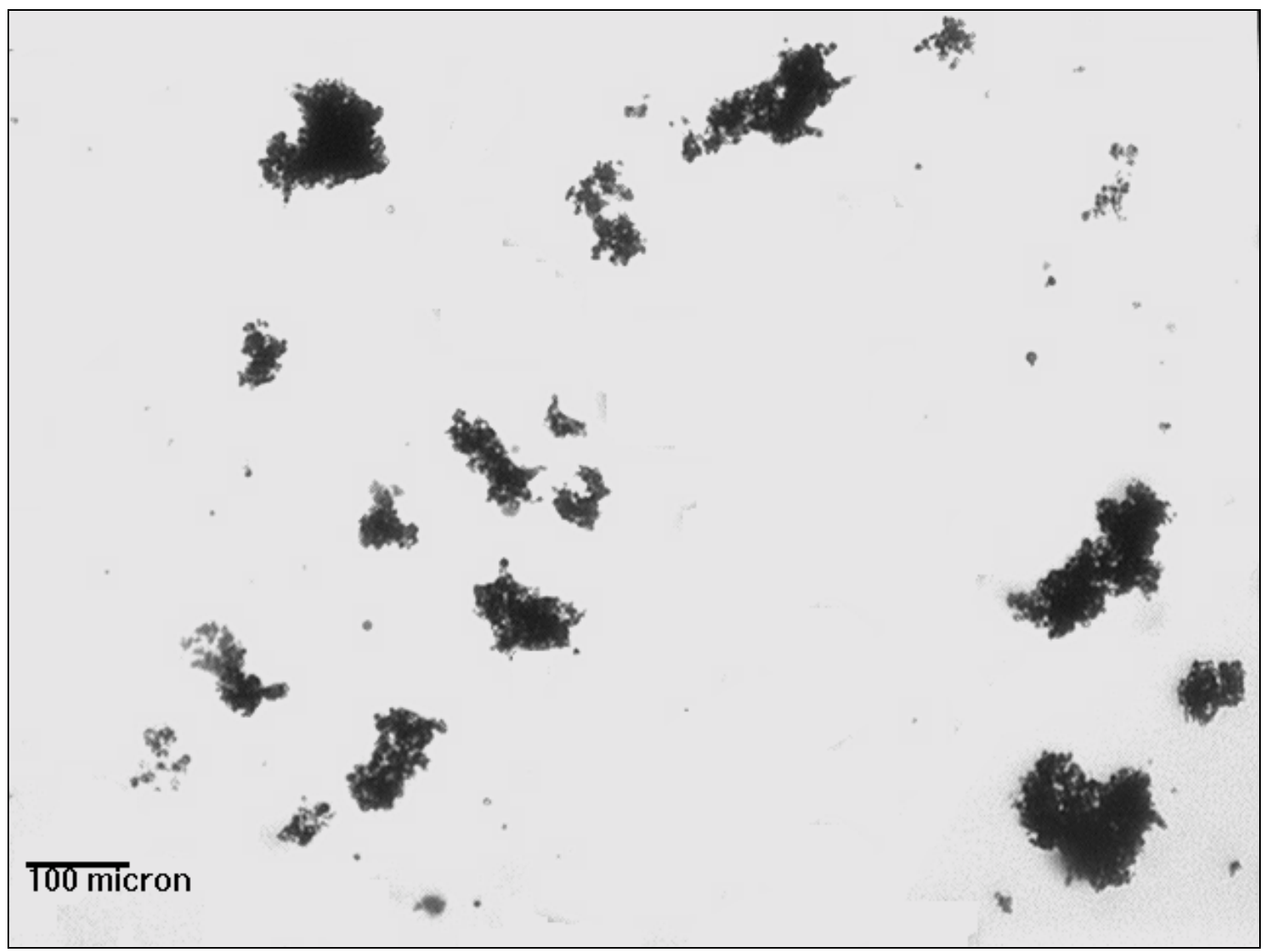

(a) 


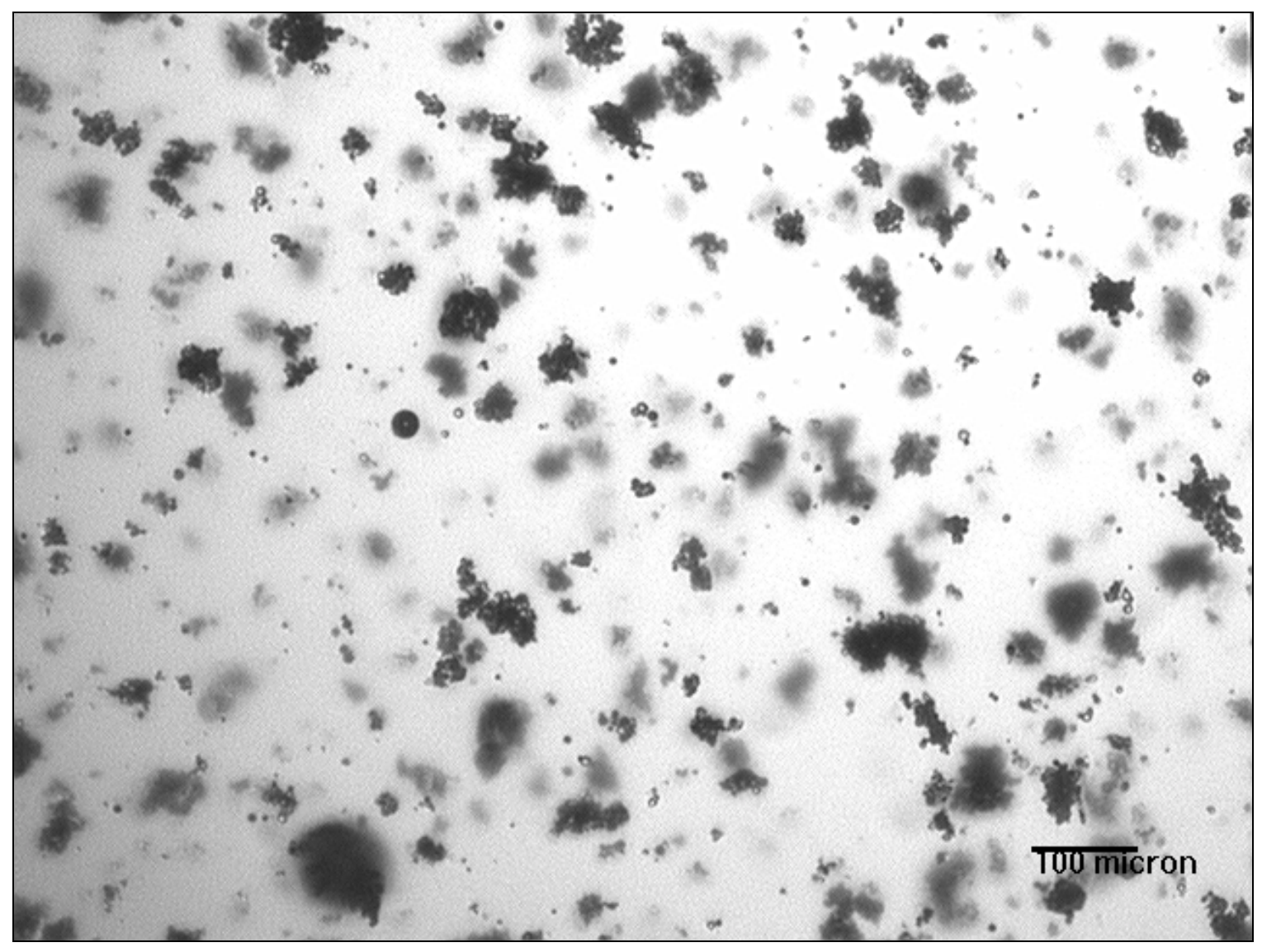

(b) 




(c)

Figure 6.8. Images of agglomerates at equilibrium formed at (a) 20 (b) 80 and (c) $190 \mathrm{~s}^{-1}$. Solid content $=0.2 \% \mathrm{v} / \mathrm{v}$, PEO conc. $=100 \mathrm{mg} / \mathrm{l}, \mathrm{MW}=1 \times 10^{6}$. 


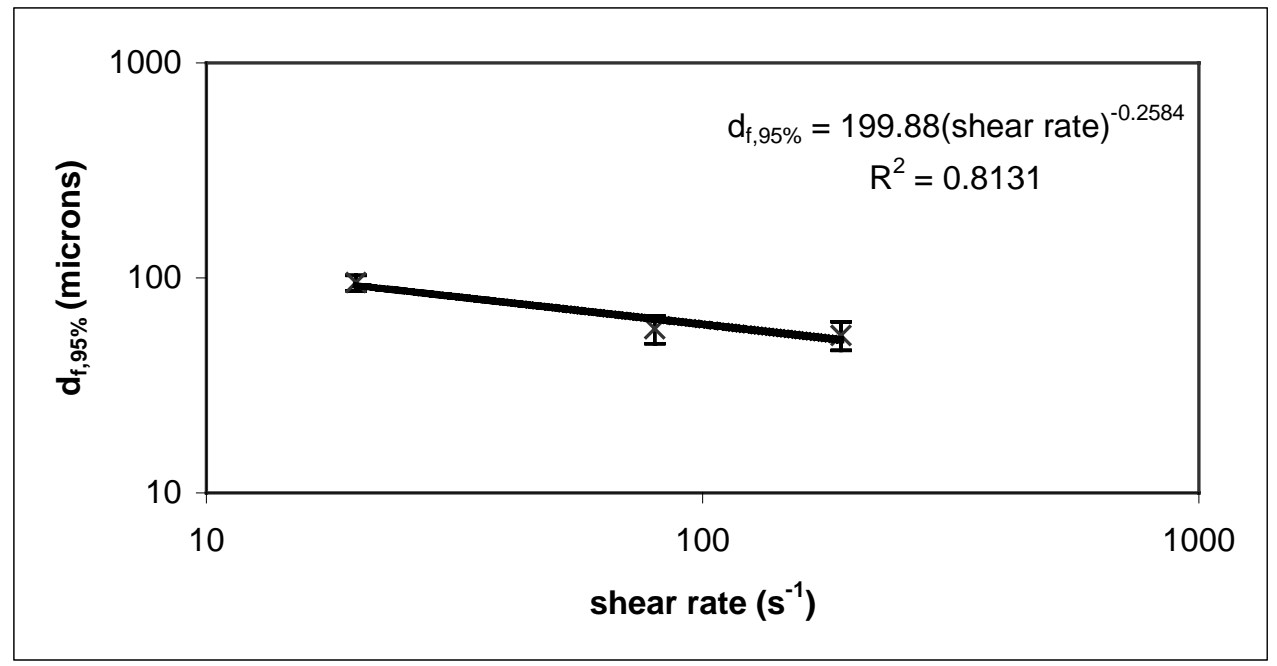

Figure 6.9. Effect of shear rate on maximum agglomerate size. Solid content $=0.2 \% \mathrm{v} / \mathrm{v}$, PEO conc. $=100 \mathrm{mg} / \mathrm{l}, \mathrm{MW}=1 \times 10^{6}$. 


\subsection{Effect of Molecular Weight of PEO on Agglomerate Size}

Molecular weight of the flocculant has very significant effect on agglomerate size. To observe this effect agglomeration experiments were performed with PEO of molecular weights of $2 \times 10^{5}, 1 \times 10^{6}$ and $5 \times 10^{6}$. Figure (6.10) shows the agglomerate growth as a function of MW of PEO at a constant rate of shearing and a constant polymer concentration.

Agglomerates grow quite large when very high molecular weight, $5 \times 10^{6}$, PEO is used. In fact, agglomerates grow so large and become so few in numbers that it is difficult to count enough of them to get a satisfactory average size, hence a large spread in average values is obtained. Agglomerates produced using lower molecular weight PEO are much smaller. This is more clearly demonstrated in Figure (6.11) where maximum agglomerate size has been plotted against the molecular weight of flocculant. Agglomerates produced by high molecular weight polymer are much stronger than those produced by lower molecular weight polymer, and hence they can withstand higher shear stresses that tend to break them up. Larger polymer molecules provide more points of contact between the bridging particles resulting in a stronger bond. Also, high molecular weight polymer molecules have a stronger segment-solid surface interaction because larger molecules tend to separate out first from a bad solvent. Muhle [54] found that adhesive strength of flocs increases significantly as the molecular weight exceeds $10^{6}$ when using hydrolyzed polyacrylamide (HPAM) as flocculant with glass beads and so does the corresponding maximum floc size. There is not much increase in floc strength when HPAM molecular weight changed from about $2 \times 10^{5}$ to $10^{6}$, but there is a steep increase in floc strength when molecular weight is increased further. This implies that 




Figure 6.10. Agglomerate growth when using different molecular weight PEO. Shear rate $=80 \mathrm{~s}^{-1}$, solid content $=0.2 \% \mathrm{v} / \mathrm{v}$, PEO concentration $=100 \mathrm{mg} / 1$. 


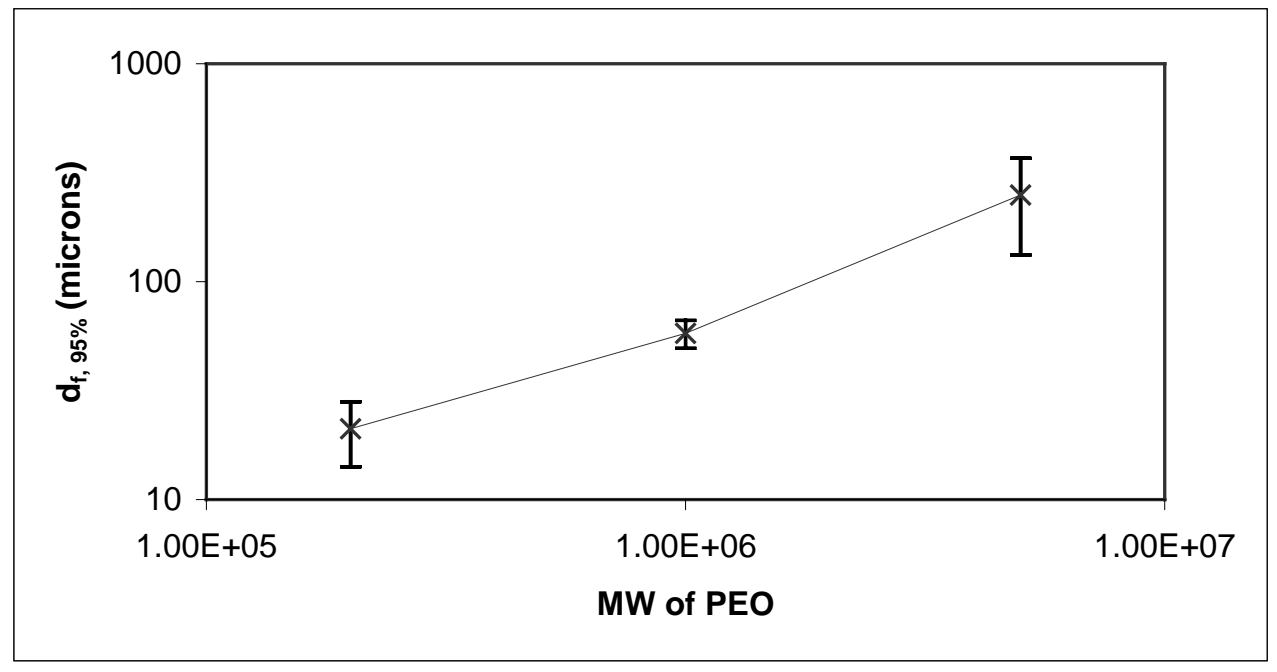

Figure 6.11. Effect of MW of PEO on maximum agglomerate size.

high molecular weight polymers are more effective flocculants than low molecular weight polymers and their effectiveness increases rapidly with the molecular weight. That's why commercial flocculant often have molecular weight in the neighborhood of $10^{7}$

\subsection{Agglomerate Breakage}

As was shown earlier, large agglomerates are produced at low shear rates and smaller agglomerates at high shear rates. Larger agglomerates are easier to break and therefore they can survive only in low shear rate flows which produce low stresses. If a suspension containing large agglomerates is subject to high shear rates, agglomerate breakage will occur. Agglomerate breakage can occur either by erosion or floc rupture as discussed in section (3.5).

Here large agglomerates were produced at $20 \mathrm{~s}^{-1}$ and $80 \mathrm{~s}^{-1}$ and hence were easiest to undergo breakage. Once agglomerates reached their equilibrium size, the shear rate 


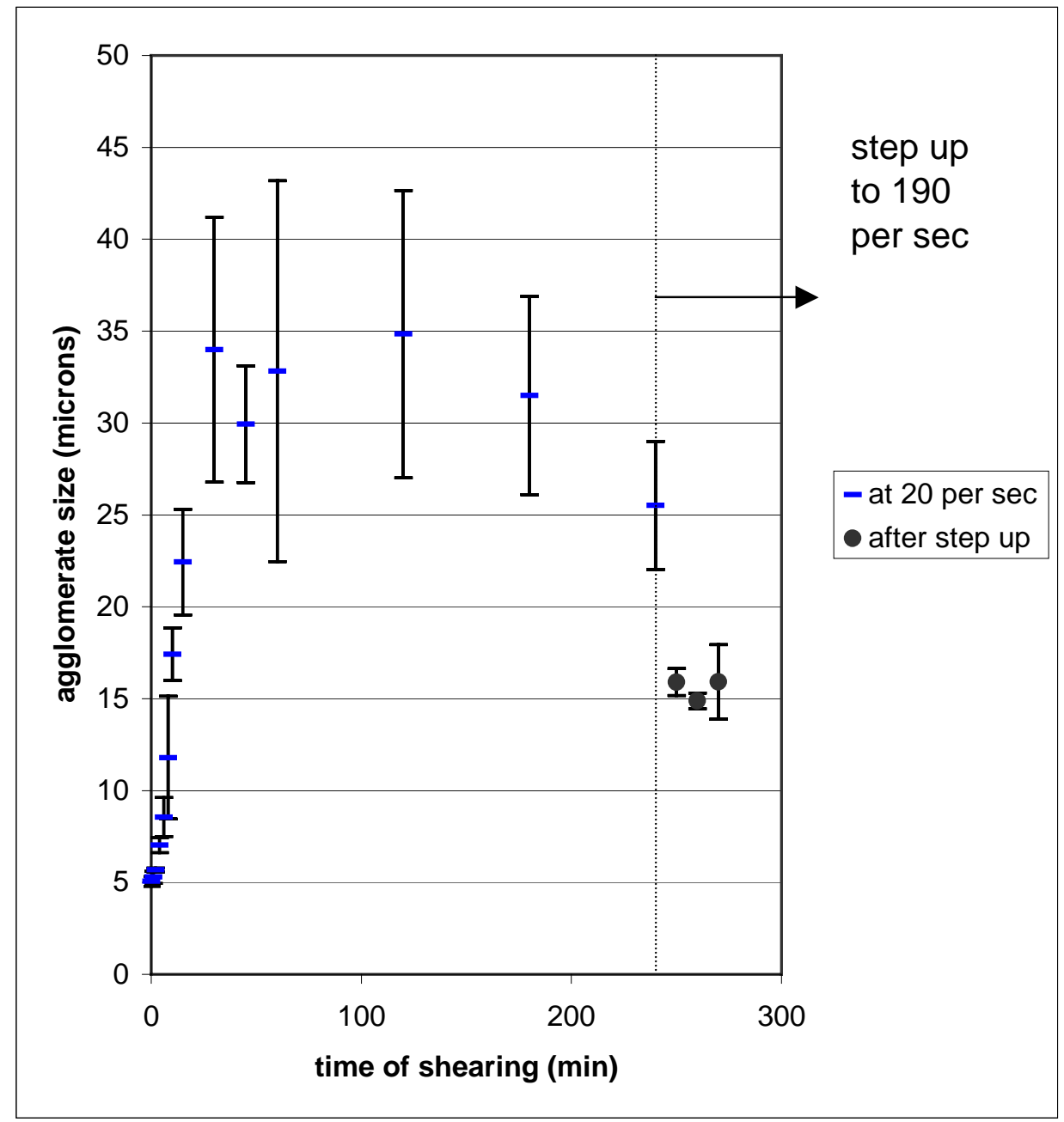

Figure 6.12. Reduction in the size of agglomerates formed at $20 \mathrm{~s}^{-1}$ after step up in shear rate. 


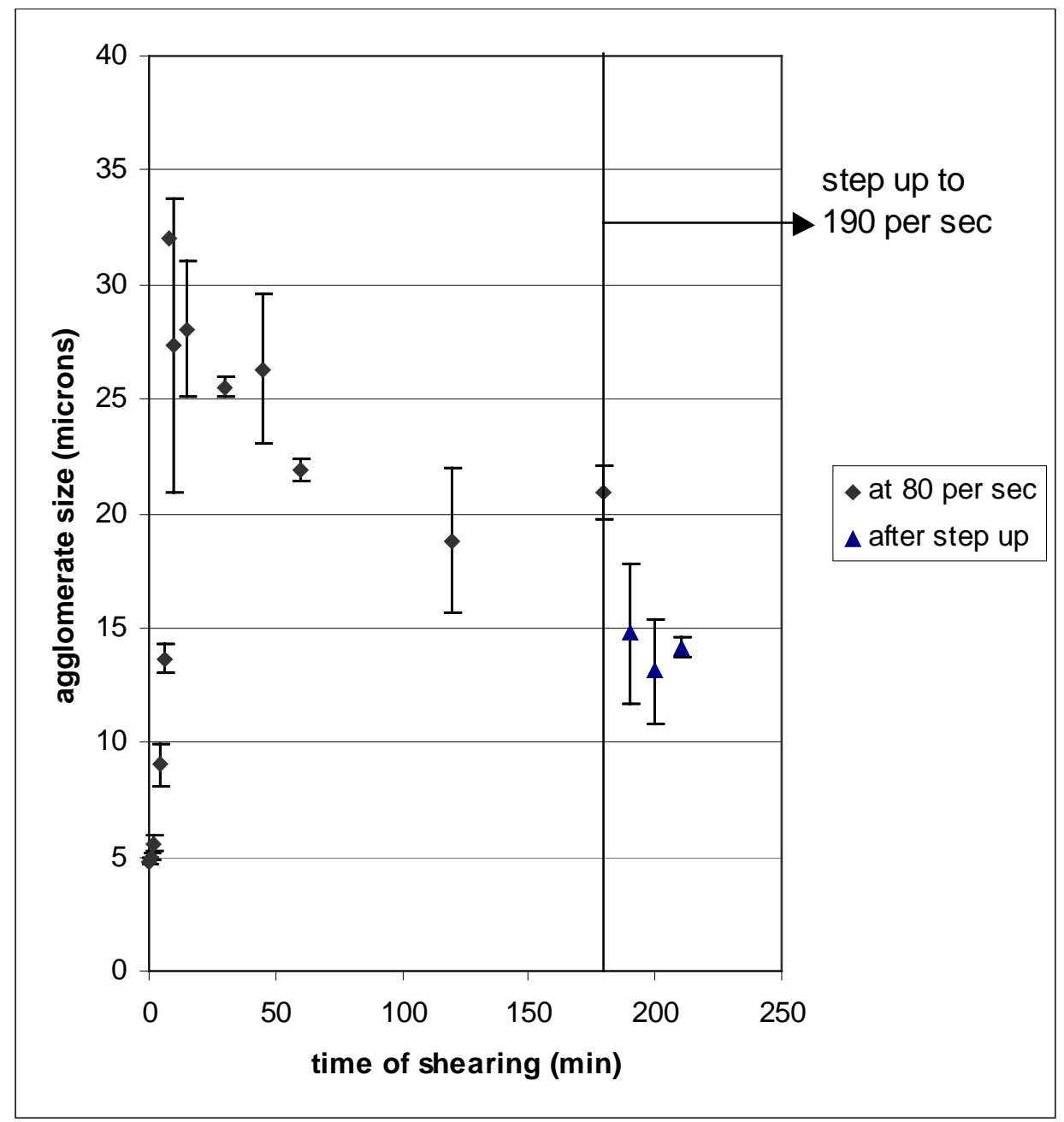

Figure 6.13. Reduction in the size of agglomerates formed at $80 \mathrm{~s}^{-1}$ after step up in shear rate. 
was suddenly stepped up to $190 \mathrm{~s}^{-1}$ and resulting agglomerate size was measured at 10 minute intervals. Agglomerate growth at $20 \mathrm{~s}^{-1}$ and $80 \mathrm{~s}^{-1}$ and then size reduction at $190 \mathrm{~s}^{-1}$ are shown in Figures (6.12) and (6.13), respectively.

As can be seen, floc breakage results in the agglomerates of smaller size which is close to that expected to exist at $190 \mathrm{~s}^{-1}$. To ascertain the floc breakage mechanism, size distribution of large agglomerates was compared to the size distribution of broken agglomerates after stepping up of shear rate. Figure (6.14) shows the size distribution of agglomerates at $20 \mathrm{~s}^{-1}$ and that after the breakage. It can be seen that the fraction of large agglomerates which are larger than $64 \mu \mathrm{m}$, decreases and the fraction of agglomerates in the intermediate range increases. This would suggest that the floc breakage is occurring via floc rupture mechanism rather than floc-erosion mechanism. Because if floc-erosion were taking place, fraction of small diameter particles close to primary particles would have increased. But that does not seem to have occurred. Inspection of digital microscope images of the pre-breakage agglomerates and post-breakage agglomerates also confirmed this because no increase in number of primary particles was observed. 


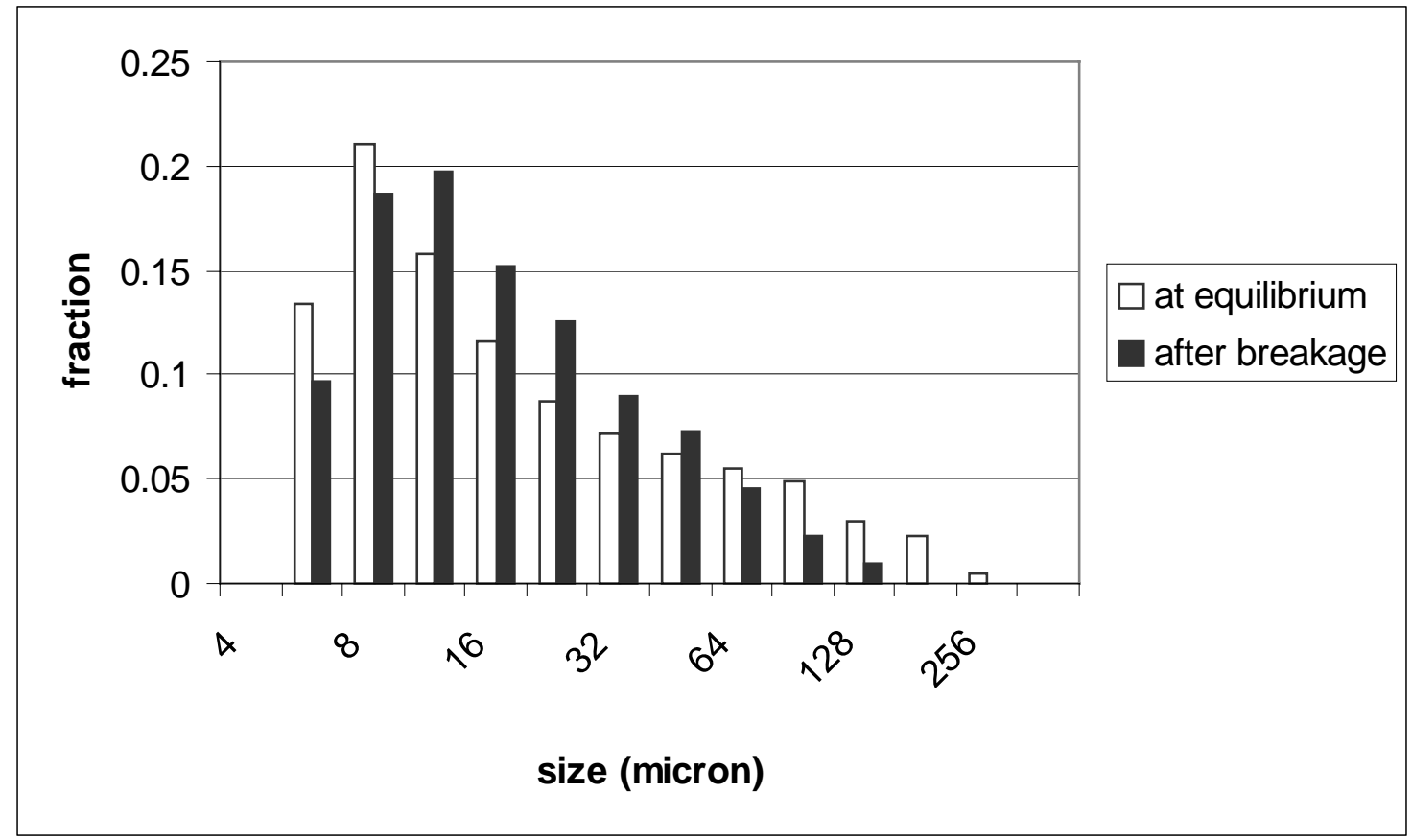

Figure 6.14. Agglomerate size distribution for the agglomerates produced at $20 \mathrm{~s}^{-1}$ and then after breakage at $190 \mathrm{~s}^{-1}$. 


\section{Chapter 7. Conclusions}

\subsection{Collision Efficiency}

In this work, a model was developed to determine the collision efficiency for the orthokinetic agglomeration process where non-colloidal particles bind together due to polymer bridging. This new model addresses some of the problems that were associated with the current methods of calculating collision efficiency in case of polymer bridging. Most of the models to calculate polymer bridging efficiency are probabilistic in nature based on the fractional surface coverage of solid particles by the flocculant. These models were developed mostly for colloidal size particles. Additionally, these models do not take into account the hydrodynamic force to include the effect of shear rate which is a very important parameter in shear-induced agglomeration.

A generalized model to calculate the collision efficiency in case of polymer bridging is proposed as given by equation (4.9). This model incorporates three possible types of interactions that occur between the agglomerating particles whose surfaces are partially covered with polymer. With the help of this model it is possible to show how the contributions of various forces to collision efficiency would change with the changing surface coverage of the particles. A formula to calculate the optimum fractional surface coverage at which the maximum collision efficiency would be obtained, was also derived from the general model and is given by equation (4.15).

For a special case where the surface of the particle is completely covered with a polymer layer, a more specific form of the collision efficiency model was derived by dimensional analysis by combining some aspects of trajectory analysis with probabilistic considerations. This model given by equation (4.27) is expressed as a function of 
dimensionless parameters which represent relative magnitudes of various forces that influence the agglomeration process, namely the van der Waals attractive force, electric double-layer repulsion, hydrodynamic force and steric force which may be attractive or repulsive. Thus, this proposed model offers improvement over other existing models as it incorporates hydrodynamic and steric effects.

Experiments were performed using a cone and plate device to determine the effects of shear rate, PEO concentration and molecular weight on the collision efficiency in the laminar flow region. Using experimental values of collision efficiency, non-linear regression analysis was employed to obtain the unknown parameters in equation (4.27). Resulting expression is equation (6.7) which can be used to evaluate the collision efficiency for the range of conditions studied in this work.

Scarce data are available in literature where effect of shear rate has been studied in conjunction with the flocculation concentration. In this work effect of shear rate on collision efficiency was studied for different level of flocculant concentration. From the experimental results, it can be concluded that the collision efficiency decreases with increasing shear rate and it can be expressed as a power-law dependence. Increasing the concentration of flocculant also results in the increase in collision efficiency. This result is specific to this study because steric interaction is attractive in nature. If steric interaction were to cause repulsion, increasing concentration would lead to diminishing collision efficiency and ultimately to steric stabilization. Results from the study of the effect of molecular weight of polymer show that high molecular weight polymers are more effective in causing the agglomeration. This can be ascribed to fact that high molecular weight polymer molecules are larger in size and hence form a thicker layer 
around the solid particles which makes it easier for the particles to come together and form a bridge between them.

\subsection{Agglomerate Size}

Growth of agglomerates was followed at different agglomeration conditions to observe the effect of variables on the equilibrium agglomerate size. In general, as the agglomeration progresses, particles combine to form large aggregates which grow quickly initially and then reach a plateau corresponding to an equilibrium size. At high shear rate equilibrium is reached more quickly than at low shear rate. As expected from earlier studies, maximum size attained by the agglomerates depends on the shear rate. Smaller agglomerates are produced at high shear rate. Maximum agglomerate size can be related to the shear rate by a power-law equation. In this study the maximum agglomerate size was found to be proportional to $\dot{\gamma}^{-0.258}$. This relationship is very similar to the ones reported by other workers in the literature. Size of the agglomerates is also determined by the strength of bonding between the particles. This was illustrated by monitoring the agglomerate size for different molecular weights of PEO which showed that high molecular weight PEO produces much larger size agglomerates. Large molecules of PEO bind particles together much strongly because they provide many more points of bridging between the particle surfaces. Therefore, agglomerates can sustain higher stresses and thus can grow bigger in size.

Agglomerate breakage studies were also performed to determine the mechanism of agglomerate breakage. Large agglomerates produced at low shear rates were subject to stepped up shear rate to cause the breakage. Comparison of size distributions of unbroken 
and broken agglomerates showed that the fraction of large agglomerates decreased but there was increase in the fraction of fine particles. From which it was concluded that in this case mechanism of agglomerate breakage was floc-rupture rather than surfaceerosion of primary particles. 


\section{Chapter 8. Recommendations for Future Work}

Like any other research endeavor, the end of this work is also left with some unanswered questions and avenues for new research. Based on the results discussed in previous chapter few suggestions are discussed below.

The collision model developed in this work incorporates all interactions between two particles as their surface coverage changes from $\theta=0$ to $\theta=1$. But it also has unknown exponents. These exponents were determined for the case where $\theta=1$. It is suggested that the collision efficiencies should be determined at PEO concentration levels for which $0<\theta<1$. In this case the particles will adhere to each other when a covered surface approaches either a bare surface or another covered surface. For the latter situation exponents are already known. Using these new collision efficiencies, exponents of the $\theta(1-\theta)$ term can be determined. The term representing bare-bare surface interaction can still be neglected as it does not result in agglomeration.

For the experimental conditions studied, the surface potential of the solid particles was constant. However, it has a significant effect on the collision efficiency as it influences the electric double-layer repulsion- higher the surface potential, larger the repulsion. The surface potential can be changed by changing the $\mathrm{pH}$ of the suspension. The surface potential can be determined by electrophoretic mobility experiments.

The steric interaction between the polymer layers depends on their thickness. The thickness of the adsorbed polymer layer was estimated from the size of polymer molecules. It is suggested that the layer thickness should be experimentally determined by techniques such as photon correlation spectroscopy. However, this would require that 
the solid particles be monodisperse. Use of monodisperse polyethylene oxide would also be advisable.

It would be interesting to study the effect of quality of solvent on the agglomeration process. As was shown in this work, $90 \%$ glycerin $+10 \%$ water solution is a "bad" solvent for PEO. Though the solvency of PEO in glycerin solution can be improved by increasing the water content, it would also considerably decrease the viscosity of the solvent and it would be difficult to decouple the effects of quality of solvent and viscosity. However, quality of the solvent can be further worsened by adding an electrolyte such as $\mathrm{NaCl}$ or $\mathrm{MgSO}_{4}$, without affecting the viscosity. Lowering the quality of solvent will be manifested as shrinkage in polymer molecule size. Consequently, thinner polymer layers will form around the solid particles, but at the same time the steric interaction will be much stronger when the polymer layers do overlap. These are two opposing effects- thinner polymer layer diminishes the collision efficiency where as low solvency of polymer promotes it. One can also expect the agglomerates formed under these conditions to be much stronger and hence bigger in size.

Another aspect that needs further exploration is the morphology of the agglomerates. Fractal geometry can be used to quantify the irregular shape of agglomerates. A regular solid object such as a sphere has fractal dimension of 2 in 2-dimensional space. Using a microscope, fractal dimension is measured in 2 dimensional space because only a 2 dimensional projected area of a 3-dimensional object is visible. An irregular shape object such as agglomerate has fractal dimension of less than 2. More open or "frilly" the agglomerate lesser would be the value of fractal dimension. The fractal dimension of the agglomerates can be related to the porosity as well. Knowledge of the fractal dimension 
and the porosity would also help in relating interparticle bridging strength with the agglomerate size. Lesser the fractal dimension, more open its structure and it becomes easy for the agglomerate to break under stress. Fractal geometry of agglomerates formed by Brownian motion is well studied. In this case agglomerates of different morphology can be obtained by altering the agglomeration conditions such as the surface potential and the temperature. It would be interesting to observe the fractal geometry of agglomerates formed by polymer bridging as not much information is available in literature. It is suggested that the molecular weight of the flocculant and the polymer solvency may have effect on the fractal geometry of the agglomerates. 


\section{Chapter 9. References}

1. Geisbers, M., J. M. Kleijn, G. J. Fleer, M. A. Cohen Stuart, "Forces between Polymer-Covered Surfaces: A Colloidal Probe Study," Colloids and Surfaces A: Physicochemical and Engineering Aspects, 142, 343-353 (1998).

2. Hiemenz, P. C., " Priciples of Colloid and Surface Chemistry," Marcel Dekker, Inc., New York (1986).

3. Swift, D. L. and S. K. Friedlander, "The Coagulation of hydrosols by Brownian Motion and Laminar Shear Flow," J. Coll. Sci., 19, 621-647 (1964).

4. Heimenz, P. C.," Principles of Colloid and Surface Chemistry," Chapter $4,2^{\text {nd }}$ ed., Marcell Dekker, Inc., New York, New York (1986).

5. Ives, K. J., "Rate Theories," in " The Scientific Basis of Flocculation" edited by K. J. Ives, Sijthoff and Noordhoff International Publishers B. V., Alphen aan Den, Rijn, 37-61(1978).

6. Kramer, T. A. and Mark M. Clark, "Influence of Strain-Rate on Coagulation Kinetics," J. Env. Engg., May, 444-452 (1997).

7. Han, M. and D. F. Lawler, "The Relative Insignificance of G in Flocculation," J. Amer. Wat. Works Assoc., Oct., 79-91 (1992).

8. Zeichner, G. R. and W. R. Schowalter, "Use of Trajectory Analysis to Study Stability of Colloidal Dispersion in Flow Fields," AIChE J., 23, 3, 243-254 (1977).

9. Hunter, R. J., "Foundations of Colloid Science, vol. 1," Chapter 4, $2^{\text {nd }}$ ed., Clarendon Press, Oxford (1989). 
10. Hunter, R. J., "Foundations of Colloid Science, vol. 1," Chapter 7, $2^{\text {nd }}$ ed., Clarendon Press, Oxford (1989).

11. Batchelor, G. K. and J. T. Green, "The Hydrodynamic Interaction of Two Small Freely Moving Spheres in a Linear Flow Field," J. Fluid Mech., 56, 2, 375-400 (1972).

12. Van de Ven, T. G. M. and S. G. Mason, "The Microrheology of Colloidal Dispersions IV. Pairs of Interacting Spheres in Shear Flow," J. Coll. Int. Sci., 57, 3, 505-516 (1976).

13. Van de Ven, T. G. M. and S. G. Mason, "The Microrheology of Colloid Dispersions VII. Orthokinetic Doublet Formation of Spheres," Colloid and Polymer Sci., 255, 468-479 (1977).

14. Gregory, J., "Polymer Flocculation in Flowing Suspension," in "The Effect of Polymers on Dispersion Properties" edited by Th. F. Tadros, Academic Press, London, 301-321(1982).

15. Greene, M. R., D. A. Hammer and W. L. Olbricht, "The Effect of Hydrodynamic Flow Field on Colloidal Stability," J. Coll. Int. Sci., 167, 232-246 (1994).

16. Adler, P. M., "Interaction of Unequal Spheres 1. Hydrodynamic InteractionsColloid Forces," J. Coll. Int. Sci., 84, 2, 461-474 (1981).

17. De Boer, G. B. J. and C De Weerd, D. Thoenes, "Coagulation in Turbulent Flow Part II," Chem. Engg. Res. Des., 67, 308-315 (1989).

18. Brakalov, L. B., "A Connection Between the Orthokinetic Coagulation Capture Efficiency of Aggregates and their Maximum Size," Chem. Engg. Sci., 42, 10, 2373-2383 (1987). 
19. Potanin, A. A., "On the Mechanism of Aggregation in the Shear Flow of Suspensions," J. Coll. Int. Sci., 145, 1, 140-157 (1991).

20. Gregory, J., "Effects of Polymers on Colloid Stability" in " The Scientific Basis of Flocculation" edited by K. J. Ives, Sijthoff and Noordhoff International Publishers B. V., Alphen aan Den, Rijn, 101-130 (1978).

21. Gregory, J., "Polymer Adsorption and Flocculation in Sheared Suspensions," Coll. and Surf., 31, 231-253 (1988).

22. Smellie, R. H., Jr. and V. K. La Mer,"Flocculation, Subsidence and Filtration of Phophate Slimes VI. A Quantitative Theory of Filtration of Flocculated Suspensions," J. Coll. Sci., 23, 589-599 (1958).

23. Hogg, R., "Collision Efficiency Factors for Polymer Flocculation," J. Coll. Int. Sci., 102, 1, 232-236 (1984).

24. Moudgil, B. M., B. D. Shah and H. S. Soto, "Collision Efficiency Factors in Polymer Flocculation of Fine Particles," J. Coll. Int. Sci., 119, 2, 466-473 (1987).

25. Dickinson, E. and L. Eriksson, "Particle Flocculation by Adsorbing Polymers," Adv. Coll. Int. Sci., 34, 1-29 (1991).

26. Aunins, J. G., "Induced Flocculation of Animal Cells in Suspension Culture," Ph. D. Thesis, Chem. Engg. Dept., Massachusetts Institute of Technology (1989).

27. Van de Ven, T. G. M., "Effect of Polymer Bridging on Selective Shear Flocculation," J. Coll. Int. Sci., 81, 1, 290-291 (1981).

28. Deason, D. M., "Statistical Model for Bridging Efficiency in Polymeric Flocculation," in "Flocculation in Biotechnology and Separation Systems" edited by Y. A. Attia, Elsevier Science Publishers B. V., Amsterdam, 21-30 (1987). 
29. Aunins, J. G. and D. I. C. Wang, "Experimental Collision Efficiencies of Polymer-Flocculated Animal Cells," Biotechnol. Prog., 6, 54-61 (1990).

30. Rumpf, H.," The Strength of Granules and Agglomerates," in Agglomeration edited by W. A. Knepper, Interscience Publishers, New York, 379-418 (1962).

31. Manas-Zloczower, I. and D. L. Feke, "Analysis of Agglomerate Rupture in Linear Flow Fields," Int. Pol. Proc. IV, 1, 3-8 (1989).

32. Boller, M. and S. Blaser, "Particles under Stress," Wat. Sci. Tech, 37, 10, 9-29 (1998).

33. Sonntag, R. C. and W. B. Russel, "Structure and Breakup of Flocs Subjected to Fluid Stresses I. Shear Experiments," J. Coll. Int. Sci., 113, 2, 399-413 (1986).

34. Oles, V., "Shear-Induced Aggregation and Breakup of Polystyrene Latex Particles," J. Coll. Int. Sci., 154, 2, 351-358 (1992).

35. Muhle, K. and K. Domasch, "Stability of Particle Aggregates in Flocculation with Polymers," Chem. Engg. Proc., 29, 1-8 (1991).

36. Shamlou, P. A. and N. Titchener-Hooker, "Turbulent Aggregation and Breakup of Particles in Liquids in Stirred Vessels," in "Processing of Solid-Liquid Suspensions" edited by P. A. Shamlou, Butterworth-Heinemann Ltd, Oxford, 125 (1993).

37. Glasgow, L. A. and J. P. Hsu, "An Experimental Study of Floc Strength," AIChE J., 28, 5, 779-785 (1982).

38. Pandya J. D. and L. A. Spielman, " Floc Breakage in Agitated Suspensions: Theory and Data Processing Strategy," J. Coll. Int. Sci., 90, 2, 517-531 (1982). 
39. Ray, D. T. and R. Hogg, "Agglomerate Breakage in Polymer-Flocculated Suspensions," J. Coll. Int. Sci., 116, 1, 256-268 (1987).

40. Lu, C. F. and L. A. Spielman, "Kinetics of Floc Breakage and Aggregation in Agitated Liquid Suspensions, " J. Coll. Int. Sci., 103, 1, 95-105 (1985).

41. Haarhoff, J. and Hannes Joubert, "Determination of Aggregation and Breahup Constants During Flocculation," Wat. Sci. Tech., 36, 4, 33-40 (1997).

42. Peng, S. J. and R. A. Williams, "Direct Measurement of Floc Breakage in Flowing Suspensions,' J. Coll. Int. Sci, 166, 321-332 (1994).

43. Spicer, P. T., S. E. Pratsinis, M. D. Trennepohl, G. H. M. Meesters, "Coagulation and Fragmentation: The Variation of Shear Rate and Time Lag for Attainment of Steadystate," Ind. Eng. Chem. Res., 35, 3074-3080 (1996).

44. Koh, P. T. L., J. R. G. Andrews and P. H. T. Uhlherr, "Modelling ShearFlocculation by Population Balances," Chem. Engg. Sci., 42, 2, 353-362 (1987).

45. Tambo, N. and Y. Watanabe, "Physical Aspects of Flocculation Process- I. Fundamental Treatise," Wat. Res., 13, 429-439 (1979).

46. Flory, P. J., " Principles of Polymer Chemistry," Chapter 12, Cornell University Press, Ithaca, New York (1953).

47. Tadros, Th. F., "Polymer Adsorption and Dispersion Stability" in "The Effect of Polymers on Dispersion Properties" edited by Th. F. Tadros, Academic Press, London, 1-38 (1982).

48. Cowell, C. and B. Vincent, "The Stability of Polystyerene Lattices in the Presence of Poly ethylene oxide" in "The Effect of Polymers on Dispersion Properties" edited by Th. F. Tadros, Academic Press, London, 263-284 (1982). 
49. De Witt, J. A. and T. G. H. Van De Ven, "The Effect of Neutral Polymers and Electrolytes on the Stability of Aqueous Polystyrene Latex," Adv. Coll. Int. Sci., 42, 41-64 (1992).

50. Smith, D. K. W. and J. A. Kitchener, "The Strength of Aggregates Formed in Flocculation," Chem. Engg. Sci., 33, 1631-1636 (1978).

51. Chang, S. H. and I. J. Chung, "Effect of Shear Flow on Polymer Desorption and Latex Dispersion stability in the Presence of Adsorbed Polymer," Macromolecules, 24, 567-571(1991).

52. Lapcik, L. B. A. and T. G. M. Van de Ven, "Effect of Polyethylene oxide on the Stability and Flocculation of Clay Dispersion," J. of Pulp and Paper Sci., 21, 1, J19-J24 (1995).

53. Polverari, M. and T. G. M. Van de Ven, "Dynamic Light Scattering of Suspensions of PEO-coated Latex Particles," Colloids and Surfaces A. Physicochemical and Engg. Aspects, 86, 209-228 (1994).

54. Muhle, K., "Floc Stability in Laminar and Turbulent Flow" in "Coagulation and Flocculation, Theory and Applications" edited by B. Dobias, Marcel Dekker, Inc., New York, 355-389 (1993).

55. Takamura, K., H. L. Goldsmith and S. G. Mason, "The Microrheology of Colloidal Dispersions XIII. Trajectories of Orthokinetic Pair Collisions of Latex Spheres in a Cationic Polyelectrolyte," J. Coll. Int. Sci., 82, 1, 190-202 (1981).

56. Strenge, K., "Structure Formation in Disperse Systems," in"Coagulation and Flocculation, Theory and Applications", edited by B. Dobias, Marcel Dekker, Inc., New York, 265-320 (1993). 
57. Ottewill, R. H. and T. Walker, "The Influence of Non-Ionic Surface Active Agents on the Stability of Polystyrene Latex Dispersions," Kolloid-Zeischrift und Zeitschrift fur Polymere, Band 227, Heft 1-2, 108-116 (1967).

58. Bailey Jr., F. E. and J. V. Koleske, "Poly (ethylene oxide)," Chapter 4, Academic Press, New York (1976).

59. Williams, R. A., S. J. Peng and A. Naylor, "In Situ Measurement of Particle Aggregation and Breakage Kinetics in a Concentrated Suspension," Powder Technology, 73, 75-83 (1992).

60. Jefferey, G. C. and R. H. Ottewill, "Reversible Aggregation Part 2. Kinetics of Reversible Aggregation," Coll. Poly. Sci., 268, 179-189 (1990).

61. Dondos, A. and H. Benoit, "The Relationship between the Unperturbed Dimensions of Polymers in Mixed Solvents and the Thermodynamic properties of the Solvent Mixture," Macromolecules, 6, 2, 242-245 (1973).

62. Flory, P. J. and T. G. Fox, Jr., "Treatment of Intrinsic Viscosities," J. Am. Chem. Soc., 73, 5, 1904-1908 (1951).

63. Vold, R. D. and M. J. Vold, "Colloid and Interface Chemistry," Addison-Wesley Publishing Company, Inc., Massachusetts (1983).

64. Elias, H., "Macromolecules.1-Structure and Properties," Plenum Press, New York (1977).

65. Attia, Y. A. and J. Rubio, "Determination of Very Low Concentration of Polyacrylamide and Polyethylene oxide Flocculants by Nephelometry," Br. Polym. J., 7, 135-138 (1975). 
66. Lyklema, J., "Fundamentals of Interface and Colloid Science, vol.1," Appendix 9, Academic Press, Inc., San Diego, CA (1991).

67. http://www.foresight.org/Nanomedicine/Ch03 3.html

68. Vial, J. and A. Carre, "Calculation of Hamaker Constant and Surface Energy of Polymers by a Simple Group Contribution Method," Int. J. Adhesion and Adhesives, 11, 3, 140-143 (1991).

69. Newman, A. A., "Glycerol," Chapter 2, C. R. C. Press, Cleveland, Ohio (1968).

70. Boller, M. and S. Blaser, "Particles under Stress," Wat. Sci. Tech, 37, 10, 9-29 (1998). 


\section{APPENDIX A}

Viscosity measurements of PEO solutions in ( $90 \%$ glycerin $+10 \%$ water)

to determine the intrinsic viscosity 
Table A1. PEO MW $=1 \times 10^{5}$, Concentration $=0.4 \mathrm{gm} / \mathrm{dl}$

\begin{tabular}{|c|c|c|}
\hline time (sec) & kinematic viscosity (cSt) & shear viscosity (poise) \\
\hline 151.03 & 175.49 & 2.16 \\
\hline 151.08 & 175.56 & 2.17 \\
\hline 151.15 & 175.64 & 2.17 \\
\hline \multicolumn{3}{|c|}{ Repeat } \\
\hline 155.58 & 180.78 & 2.23 \\
\hline 155.32 & 180.48 & 2.23 \\
\hline 155.37 & 180.54 & 2.23 \\
\hline
\end{tabular}

Table A2. PEO MW $=1 \times 10^{5}$, Concentration $=0.6 \mathrm{gm} / \mathrm{dl}$

\begin{tabular}{|c|c|c|}
\hline time (sec) & kinematic viscosity (cSt) & shear viscosity (poise) \\
\hline 172.89 & 200.89 & 2.48 \\
\hline 171.77 & 199.59 & 2.46 \\
\hline 171.17 & 198.89 & 2.45 \\
\hline \multicolumn{3}{|c|}{ Repeat } \\
\hline 173.99 & 202.18 & 2.49 \\
\hline 173.4 & 201.49 & 2.49 \\
\hline 172.88 & 200.89 & 2.48 \\
\hline
\end{tabular}

Table A3. PEO MW $=1 \times 10^{5}$, Concentration $=0.8 \mathrm{gm} / \mathrm{dl}$

\begin{tabular}{|c|c|c|}
\hline time (sec) & kinematic viscosity (cSt) & shear viscosity (poise) \\
\hline 192.36 & 223.80 & 2.76 \\
\hline 190.57 & 221.44 & 2.73 \\
\hline 188.29 & 218.79 & 2.699 \\
\hline \multicolumn{3}{|c|}{ Repeat } \\
\hline 195.53 & 227.21 & 2.80 \\
\hline 193.49 & 224.83 & 2.77 \\
\hline 192.29 & 223.44 & 2.76 \\
\hline
\end{tabular}


Table A4. PEO MW $=2 \times 10^{5}$, Concentration $=0.2 \mathrm{gm} / \mathrm{dl}$

\begin{tabular}{|c|c|c|}
\hline time (sec) & kinematic viscosity (cSt) & shear viscosity (poise) \\
\hline 148.17 & 172.17 & 2.12 \\
\hline 148.66 & 172.74 & 2.13 \\
\hline 148.85 & 172.96 & 2.13 \\
\hline \multicolumn{3}{|c|}{ Repeat } \\
\hline 149.88 & 174.16 & 2.15 \\
\hline 149.72 & 173.97 & 2.15 \\
\hline 149.45 & 173.66 & 2.14 \\
\hline
\end{tabular}

Table A5. PEO MW $=2 \times 10^{5}$, Concentration $=0.3 \mathrm{gm} / \mathrm{dl}$

\begin{tabular}{|c|c|c|}
\hline time (sec) & kinematic viscosity (cSt) & shear viscosity (poise) \\
\hline 165.58 & 192.40 & 2.37 \\
\hline 165.43 & 192.23 & 2.37 \\
\hline 165.55 & 192.37 & 2.37 \\
\hline \multicolumn{3}{|c|}{ Repeat } \\
\hline 158.21 & 183.84 & 2.27 \\
\hline 158.25 & 183.89 & 2.27 \\
\hline 158.38 & 184.04 & 2.27 \\
\hline
\end{tabular}

Table A6. PEO MW $=2 \times 10^{5}$, Concentration $=0.4 \mathrm{gm} / \mathrm{dl}$

\begin{tabular}{|c|c|c|}
\hline time (sec) & kinematic viscosity (cSt) & shear viscosity (poise) \\
\hline 172.81 & 200.8 & 2.48 \\
\hline 172.17 & 200.06 & 2.47 \\
\hline 171.68 & 199.49 & 2.46 \\
\hline \multicolumn{3}{|c|}{ Repeat } \\
\hline 176.67 & 205.29 & 2.53 \\
\hline 175.67 & 204.13 & 2.52 \\
\hline 175.58 & 203.93 & 2.52 \\
\hline
\end{tabular}

Table A7. PEO MW $=2 \times 10^{5}$, Concentration $=0.45 \mathrm{gm} / \mathrm{dl}$

\begin{tabular}{|c|c|c|}
\hline time (sec) & kinematic viscosity (cSt) & shear viscosity (poise) \\
\hline 187.06 & 217.36 & 2.68 \\
\hline 187.66 & 218.06 & 2.69 \\
\hline 187.7 & 218.11 & 2.69 \\
\hline
\end{tabular}


Table A8. Reduced Viscosity of PEO MW $=1 \times 10^{5}$

\begin{tabular}{|c|c|c|}
\hline $\mathbf{C}(\mathbf{g m} / \mathbf{d l})$ & $\boldsymbol{\eta}$ (poise) & $\frac{1}{C}\left[\frac{\eta}{\eta_{0}}-1\right](\mathbf{d l} / \mathbf{g m})$ \\
\hline 0.8 & 2.75 & 0.47 \\
\hline 0.6 & 2.47 & 0.39 \\
\hline 0.4 & 2.19 & 0.25 \\
\hline
\end{tabular}

Table A9. Reduced Viscosity of PEO MW $=2 \times 10^{5}$

\begin{tabular}{|c|c|c|}
\hline $\mathbf{C}$ (gm/dl) & $\boldsymbol{\eta}$ (poise) & $\frac{1}{C}\left[\frac{\eta}{\eta_{0}}-1\right]$ (dl/gm) \\
\hline 0.45 & 2.69 & 0.77 \\
\hline 0.4 & 2.49 & 0.62 \\
\hline 0.3 & 2.32 & 0.54 \\
\hline 0.2 & 2.14 & 0.35 \\
\hline
\end{tabular}




\section{APPENDIX B}

Agglomeration data 


\section{Table B1}

- Solid content $=0.2 \% \mathrm{v} / \mathrm{v}$

- $\mathrm{PEO}$ concentration $=100 \mathrm{mg} / \mathrm{l}$

- $\quad$ PEO MW $=5 \times 10^{6}$

- Shear rate $=80 \mathrm{~s}^{-1}$

\begin{tabular}{|c|c|c|c|c|c|c|}
\hline \multirow{2}{*}{ time (min) } & \multicolumn{2}{|c|}{ experiment \# 1} & \multicolumn{2}{|c|}{ experiment \#2 } & \multicolumn{2}{|c|}{ experiment \#3 } \\
\hline & $\mathbf{N}$ & $\operatorname{dia}(\mu \mathrm{m})$ & $\mathbf{N}$ & $\operatorname{dia}(\mu \mathrm{m})$ & $\mathbf{N}$ & dia $(\mu \mathrm{m})$ \\
\hline 0 & 817 & 4.98 & 818 & 4.84 & 871 & 4.95 \\
\hline 2 & 157 & 8.91 & 124 & 8.13 & 124 & 11.3 \\
\hline 5 & & 17.41 & & 18.33 & & 16.00 \\
\hline 15 & & 32.63 & & 32.78 & & 41.09 \\
\hline 30 & & 31.66 & & 37.85 & & 49.06 \\
\hline 45 & & 38.03 & & 33.84 & & 45.43 \\
\hline 60 & & 37.49 & & 38.82 & & 48.14 \\
\hline 120 & & 48.77 & & 47.52 & & 94.81 \\
\hline 180 & & 44.69 & & 41.88 & & 59.02 \\
\hline 240 & & 50.08 & & 51.93 & & 62.84 \\
\hline
\end{tabular}




\section{Table B2}

- Solid content $=0.2 \% \mathrm{v} / \mathrm{v}$

- $\quad$ PEO concentration $=100 \mathrm{mg} / \mathrm{l}$

- $\quad$ PEO MW $=2 \times 10^{5}$

- Shear rate $=80 \mathrm{~s}^{-1}$

\begin{tabular}{|c|c|c|c|c|c|c|}
\hline \multirow{2}{*}{ time (min) } & \multicolumn{2}{|c|}{ experiment \# 4} & \multicolumn{2}{|c|}{ experiment \#5 } & \multicolumn{2}{|c|}{ experiment \#6 } \\
\hline & $\mathbf{N}$ & $\operatorname{dia}(\mu \mathrm{m})$ & $\mathbf{N}$ & $\operatorname{dia}(\mu \mathrm{m})$ & $\mathbf{N}$ & dia $(\mu \mathrm{m})$ \\
\hline 0 & 962 & 4.93 & 910 & 4.95 & 860 & 4.93 \\
\hline 2 & 692 & 5.69 & 624 & 5.76 & 595 & 5.56 \\
\hline 5 & 293 & 7.96 & 282 & 7.56 & 319 & 7.94 \\
\hline 15 & & 14.67 & & 13.48 & & 13.2 \\
\hline 30 & & 12.27 & & 9.41 & & 10.46 \\
\hline 45 & & 12.17 & & 8.65 & & 11.15 \\
\hline 60 & & 9.65 & & 10.06 & & 10.36 \\
\hline 90 & & 9.51 & & 9.8 & & 9.56 \\
\hline 120 & & 8.49 & & 13.78 & & 8.67 \\
\hline 180 & & 9.06 & & 12.94 & & 8.40 \\
\hline 240 & & 8.63 & & 13.41 & & 7.82 \\
\hline
\end{tabular}




\section{Table B3}

- Solid content $=0.2 \% \mathrm{v} / \mathrm{v}$

- $\mathrm{PEO}$ concentration $=100 \mathrm{mg} / \mathrm{l}$

- $\mathrm{PEO} M W=1 \times 10^{6}$

- Shear rate $=20 \mathrm{~s}^{-1}$ then step up to $190 \mathrm{~s}^{-1}$

\begin{tabular}{|l|l|l|l|l|l|l|}
\hline \multirow{2}{*}{ time (min) } & \multicolumn{2}{|l|}{ experiment \# } & \multicolumn{2}{l|}{ experiment \#8 } & \multicolumn{2}{l|}{ experiment \#9 } \\
\cline { 2 - 7 } & $\mathbf{N}$ & $\mathbf{d i a}(\boldsymbol{\mu m})$ & $\mathbf{N}$ & $\mathbf{d i a}(\boldsymbol{\mu m})$ & $\mathbf{N}$ & dia $(\boldsymbol{\mu m})$ \\
\hline 0 & 767 & 5.32 & 827 & 5.04 & 886 & 4.81 \\
\hline 1 & 741 & 5.11 & 659 & 5.67 & 734 & 5.08 \\
\hline 2 & 604 & 5.77 & 583 & 5.70 & 681 & 5.56 \\
\hline 4 & 305 & 6.60 & 334 & 7.41 & 376 & 7.11 \\
\hline 6 & 168 & 9.03 & 198 & 9.31 & 205 & 7.34 \\
\hline 8 & 181 & 8.95 & & 15.48 & 146 & 10.95 \\
\hline 10 & & 15.97 & & 17.45 & & 18.84 \\
\hline 15 & & ------- & & 20.40 & & 24.46 \\
\hline 30 & & 33.40 & & 27.08 & & 41.46 \\
\hline 45 & & 32.4 & & 26.36 & & 31.05 \\
\hline 60 & & 35.41 & & 21.42 & & 41.65 \\
\hline 120 & & 35.15 & & 26.87 & & 42.50 \\
\hline 180 & & 31.66 & & 26.03 & & 36.80 \\
\hline 240 & & 27.80 & & 21.505 & & 27.24 \\
\hline up10 & & 15.07 & & 16.19 & & 16.44 \\
\hline up20 & & 14.97 & & 15.26 & & 14.41 \\
\hline up30 & & 18.22 & & 14.42 & & 15.11 \\
\hline
\end{tabular}




\section{Table B4}

- Solid content $=0.2 \% \mathrm{v} / \mathrm{v}$

- $\quad$ PEO concentration $=100 \mathrm{mg} / \mathrm{l}$

- $\quad$ PEO MW $=1 \times 10^{6}$

- Shear rate $=190 \mathrm{~s}^{-1}$

\begin{tabular}{|l|l|l|l|l|l|l|}
\hline \multirow{2}{*}{ time (min) } & \multicolumn{2}{|l|}{ experiment \# 10 } & \multicolumn{2}{l|}{ experiment \#11 } & \multicolumn{2}{l|}{ experiment \#12 } \\
\cline { 2 - 7 } & N & dia $(\mu \mathrm{m})$ & $\mathbf{N}$ & dia $(\mu \mathrm{m})$ & $\mathbf{N}$ & dia $(\mu \mathrm{m})$ \\
\hline 0 & 855 & 4.78 & 873 & 4.80 & 805 & 4.83 \\
\hline 1 & 607 & 5.83 & 738 & 5.24 & 777 & 5.28 \\
\hline 2 & 216 & 8.53 & 411 & 6.74 & 443 & 6.50 \\
\hline 4 & 78 & 14.33 & 115 & 12.86 & 109 & 13.26 \\
\hline 6 & 83 & 10.512 & 50 & 19.65 & 54 & 21.69 \\
\hline 8 & & 19.23 & & 26.10 & & 24.39 \\
\hline 10 & & 19.22 & & 24.35 & & 20.18 \\
\hline 15 & & 15.38 & & 24.91 & & 24.92 \\
\hline 30 & & 19.31 & & 20.99 & & 20.28 \\
\hline
\end{tabular}




\section{Table B5}

- Solid content $=0.2 \% \mathrm{v} / \mathrm{v}$

- $\mathrm{PEO}$ concentration $=100 \mathrm{mg} / \mathrm{l}$

- $\quad$ PEO MW $=1 \times 10^{6}$

- Shear rate $=80 \mathrm{~s}^{-1}$ then step up to $190 \mathrm{~s}^{-1}$

\begin{tabular}{|l|l|l|l|l|}
\hline \multirow{2}{*}{ time (min) } & \multicolumn{3}{|l|}{ experiment \# 13 } & \multicolumn{2}{l|}{ experiment \#14 } \\
\cline { 2 - 5 } & $\mathbf{N}$ & dia $(\boldsymbol{\mu m})$ & $\mathbf{N}$ & dia $(\boldsymbol{\mu m})$ \\
\hline 0 & 872 & 4.90 & 916 & 4.71 \\
\hline 1 & 766 & 5.08 & 880 & 4.88 \\
\hline 2 & 568 & 5.797 & 711 & 5.32 \\
\hline 4 & 220 & 9.68 & 214 & 8.38 \\
\hline 6 & 93 & 14.14 & 85 & 13.19 \\
\hline 8 & & 46.92 & & 17.13 \\
\hline 10 & & 31.92 & & 22.82 \\
\hline 15 & & 25.97 & & 30.14 \\
\hline 30 & & 25.23 & & 25.82 \\
\hline 45 & & 23.99 & & 28.64 \\
\hline 60 & & 22.27 & & 21.52 \\
\hline 120 & & 16.56 & & 21.05 \\
\hline 180 & & 20.06 & & 21.75 \\
\hline up10 & & 16.95 & & 12.61 \\
\hline up20 & & 14.75 & & 11.51 \\
\hline up30 & & 14.46 & & 13.86 \\
\hline
\end{tabular}




\section{Table B6}

- Solid content $=0.2 \% \mathrm{v} / \mathrm{v}$

- $\mathrm{PEO}$ concentration $=20 \mathrm{mg} / \mathrm{l}$

- $\quad$ PEO MW $=1 \times 10^{6}$

- Shear rate $=20 \mathrm{~s}^{-1}$

\begin{tabular}{|l|l|l|l|}
\hline time (min) & $\begin{array}{l}\text { experiment \#15 } \\
\text { N }\end{array}$ & $\begin{array}{l}\text { experiment \#16 } \\
\text { N }\end{array}$ & $\begin{array}{l}\text { experiment \#17 } \\
\text { N }\end{array}$ \\
\hline 0 & 937 & 906 & 849 \\
\hline 2 & 921 & 867 & 745 \\
\hline 4 & 793 & 885 & 714 \\
\hline 6 & 584 & 720 & 775 \\
\hline 8 & 446 & 588 & 620 \\
\hline 10 & 455 & 496 & 486 \\
\hline 12 & ---- & 429 & 374 \\
\hline
\end{tabular}

- Shear rate $=80 \mathrm{~s}^{-1}$

\begin{tabular}{|l|l|l|l|}
\hline time (min) & $\begin{array}{l}\text { experiment \#18 } \\
\mathbf{N}\end{array}$ & $\begin{array}{l}\text { experiment \#19 } \\
\mathbf{N}\end{array}$ & $\begin{array}{l}\text { experiment \#20 } \\
\mathbf{N}\end{array}$ \\
\hline 0 & 934 & 910 & 936 \\
\hline 2 & 866 & 872 & 834 \\
\hline 5 & 614 & 610 & 646 \\
\hline 10 & 316 & 272 & 297 \\
\hline
\end{tabular}

- Shear rate $=190 \mathrm{~s}^{-1}$

\begin{tabular}{|l|l|l|l|}
\hline time (min) & $\begin{array}{l}\text { experiment \#21 } \\
\text { N }\end{array}$ & $\begin{array}{l}\text { experiment \#22 } \\
\mathbf{N}\end{array}$ & $\begin{array}{l}\text { experiment \#23 } \\
\text { N }\end{array}$ \\
\hline 0 & 859 & 810 & 787 \\
\hline 2 & 878 & 840 & 823 \\
\hline 4 & 644 & 675 & 607 \\
\hline 6 & 501 & 602 & 478 \\
\hline 8 & 298 & 347 & 304 \\
\hline
\end{tabular}




\section{Table B7}

- Solid content $=0.2 \% \mathrm{v} / \mathrm{v}$

- $\quad$ PEO concentration $=200 \mathrm{mg} / \mathrm{l}$

- $\quad$ PEO MW $=1 \times 10^{6}$

- Shear rate $=20 \mathrm{~s}^{-1}$

\begin{tabular}{|l|l|l|l|}
\hline time (min) & $\begin{array}{l}\text { experiment \#24 } \\
\text { N }\end{array}$ & $\begin{array}{l}\text { experiment \#25 } \\
\text { N }\end{array}$ & $\begin{array}{l}\text { experiment \#26 } \\
\text { N }\end{array}$ \\
\hline 0 & 791 & 799 & 843 \\
\hline 1 & 464 & 354 & 503 \\
\hline 2 & 241 & 219 & 271 \\
\hline
\end{tabular}

- Shear rate $=80 \mathrm{~s}^{-1}$

\begin{tabular}{|l|l|l|l|}
\hline time (min) & $\begin{array}{l}\text { experiment \#27 } \\
\mathbf{N}\end{array}$ & $\begin{array}{l}\text { experiment \#28 } \\
\mathbf{N}\end{array}$ & $\begin{array}{l}\text { experiment \#29 } \\
\mathbf{N}\end{array}$ \\
\hline 0 & 766 & 741 & 723 \\
\hline 1 & 217 & 234 & 227 \\
\hline 2 & 327 & 102 & 129 \\
\hline
\end{tabular}

- Shear rate $=190 \mathrm{~s}^{-1}$

\begin{tabular}{|l|l|l|l|}
\hline time (min) & $\begin{array}{l}\text { experiment \#30 } \\
\text { N }\end{array}$ & $\begin{array}{l}\text { experiment \#31 } \\
\text { N }\end{array}$ & $\begin{array}{l}\text { experiment \#32 } \\
\text { N }\end{array}$ \\
\hline 0 & 859 & 810 & 787 \\
\hline 2 & 878 & 840 & 823 \\
\hline 4 & 644 & 675 & 607 \\
\hline 6 & 501 & 602 & 478 \\
\hline 8 & 298 & 347 & 304 \\
\hline
\end{tabular}




\section{APPENDIX C}

Plots of $\mathrm{N} / \mathrm{N}_{0}$ vs. $\mathrm{t}$ to determine $\mathrm{t}_{1 / 2}$ at different flocculation conditions 
(a)

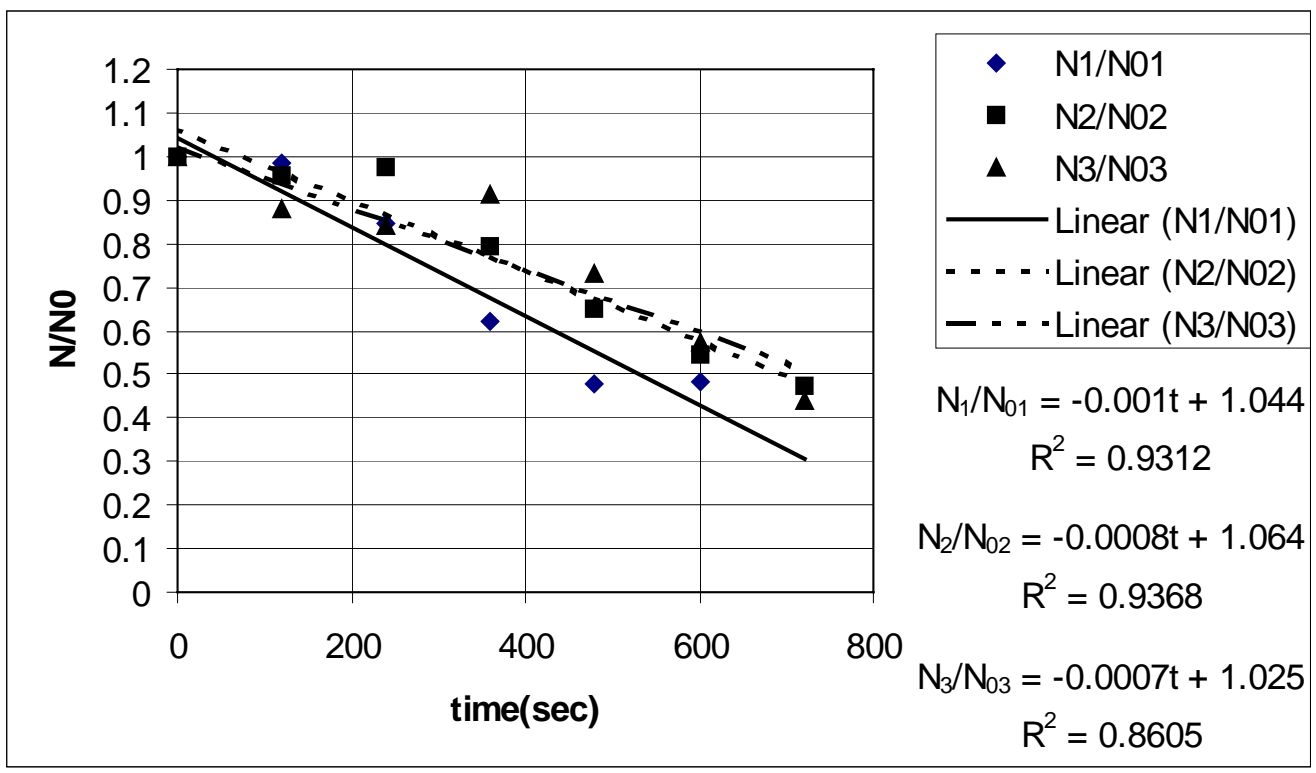

(b)




(c)

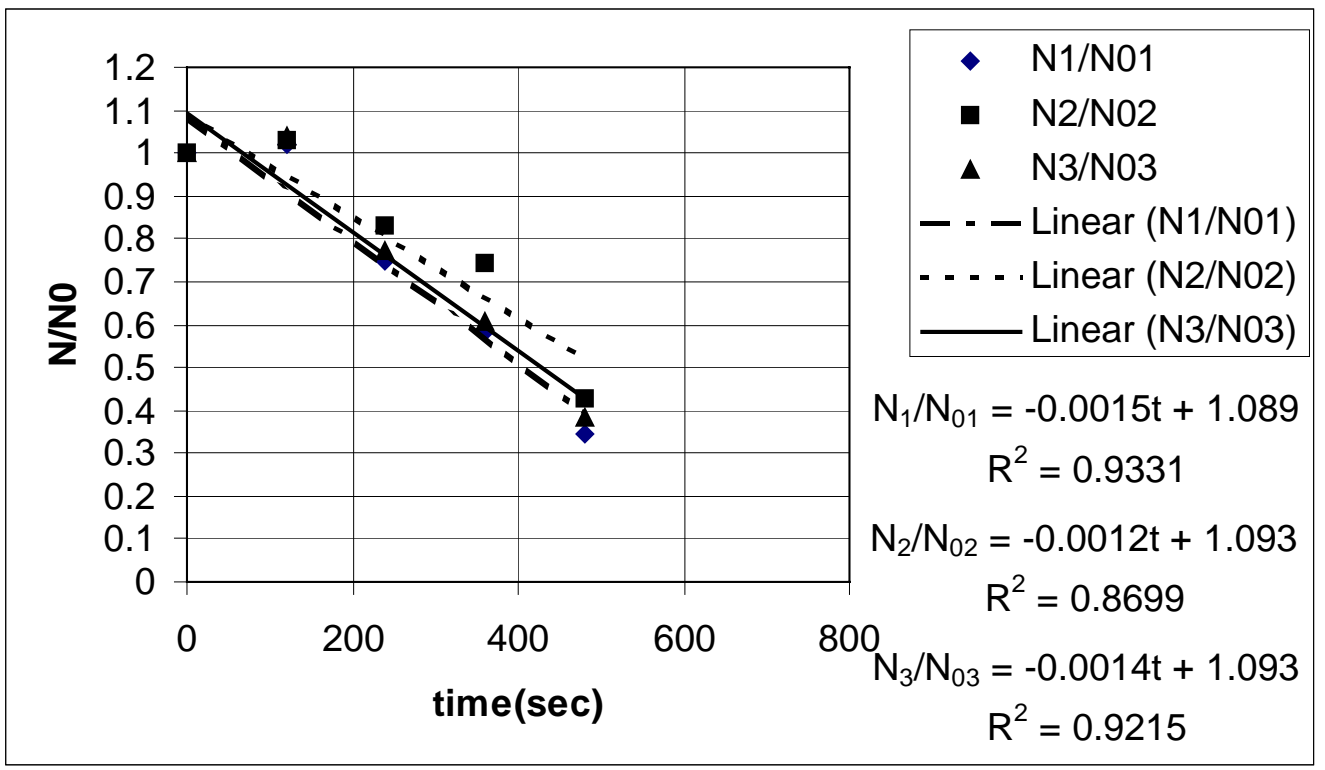

Figure C1. For PEO concentration $=20 \mathrm{mg} / \mathrm{l}$, relative particle count versus time of shearing at (a) $20 \mathrm{~s}^{-1}$ (b) $80 \mathrm{~s}^{-1}$ and (c) $190 \mathrm{~s}^{-1}$. Solid content $=0.2 \% \mathrm{v} / \mathrm{v}$, PEO MW $=1 \times$ $10^{6}$ 
(a)



(b)




(c)

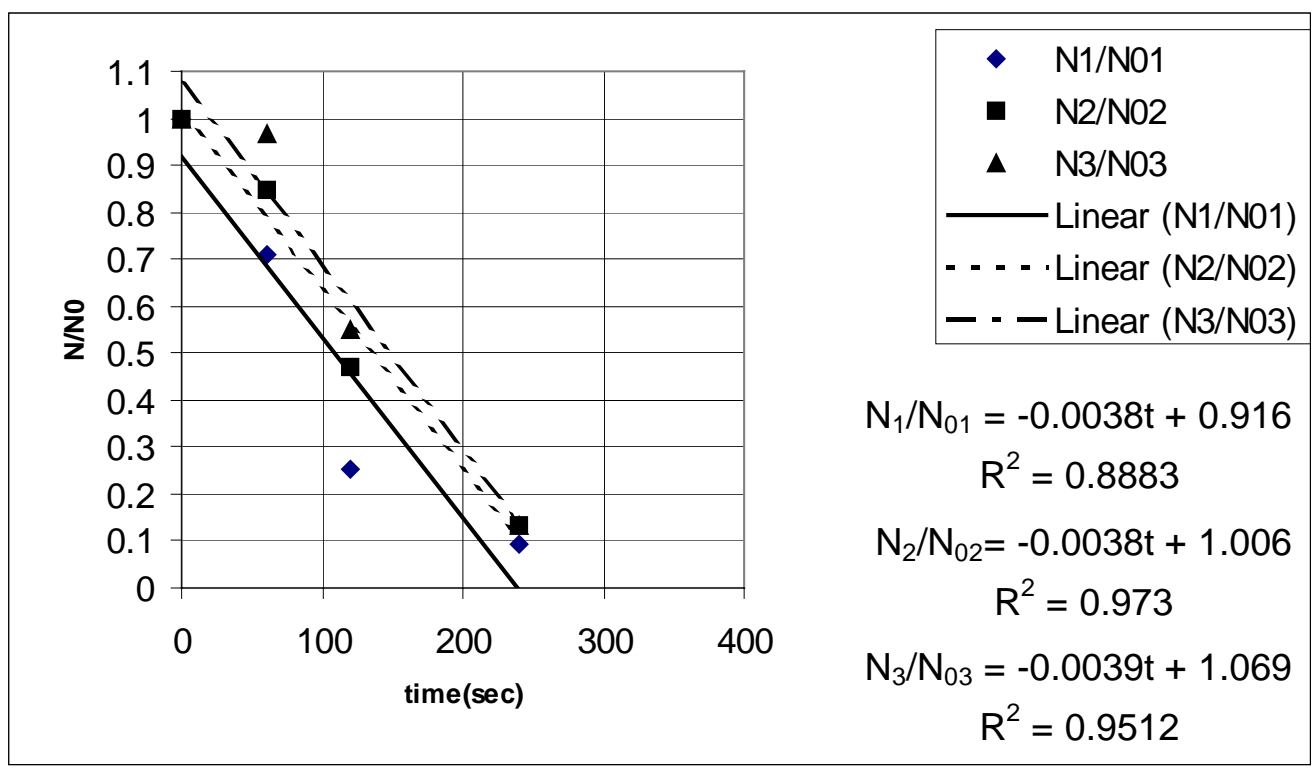

Figure C2. For PEO concentration $=100 \mathrm{mg} / \mathrm{l}$, relative particle count versus time of shearing at (a) $20 \mathrm{~s}^{-1}$ (b) $80 \mathrm{~s}^{-1}$ and (c) $190 \mathrm{~s}^{-1}$. Solid content $=0.2 \% \mathrm{v} / \mathrm{v}$, PEO MW $=1 \times$ $10^{6}$ 
(a)

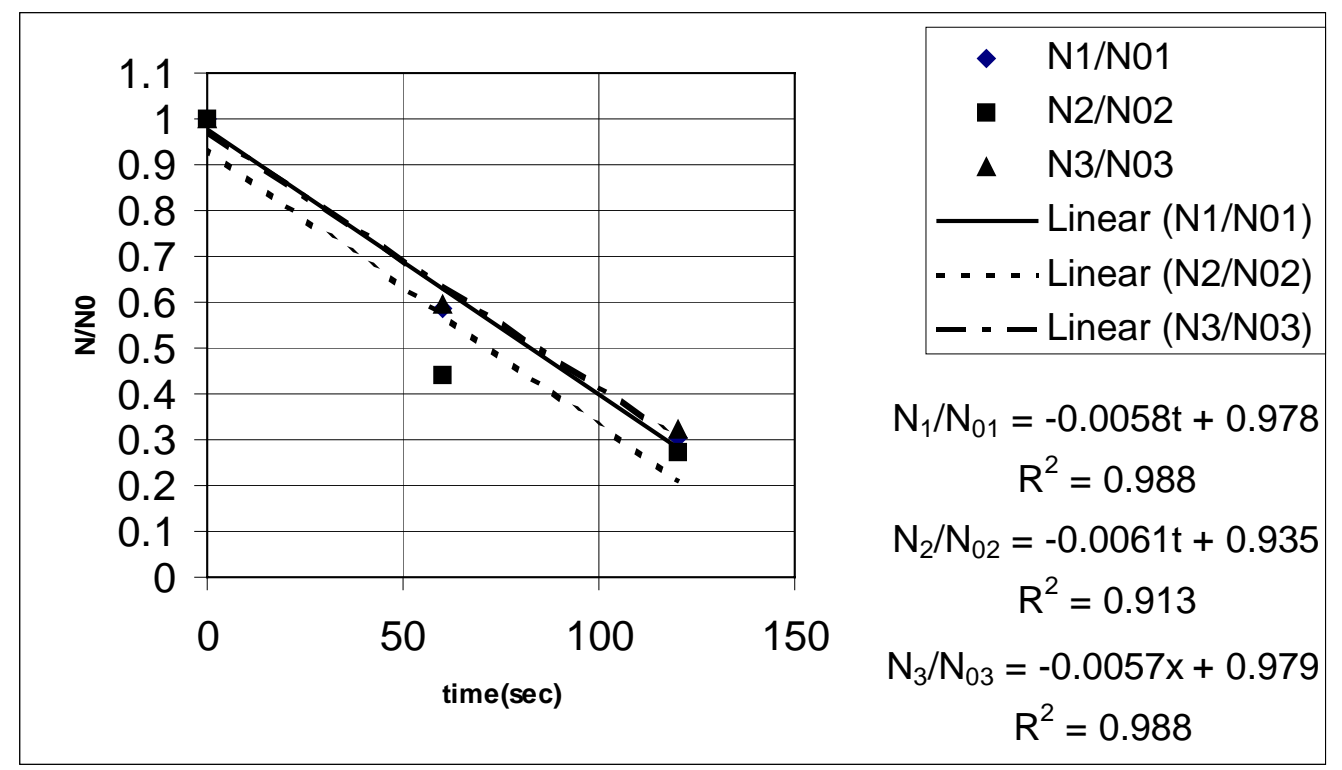

(b)

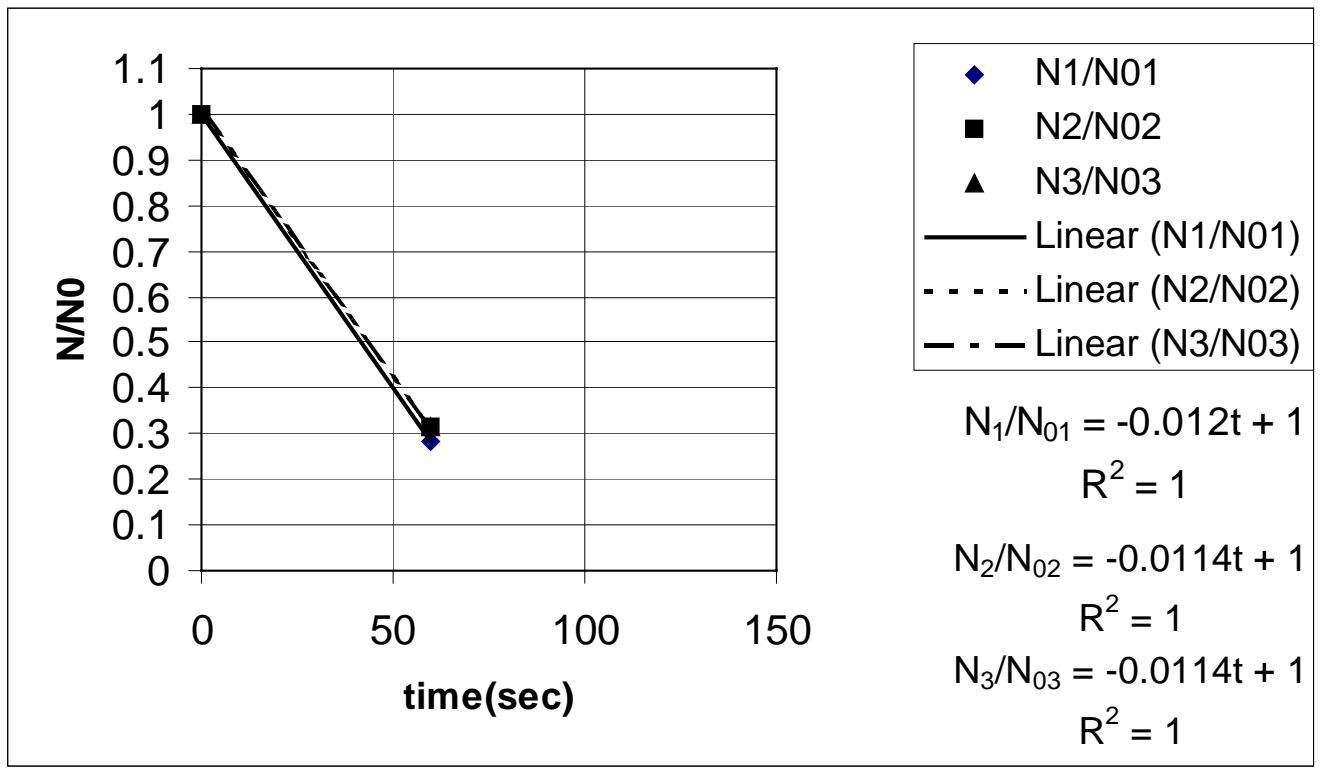


(c)

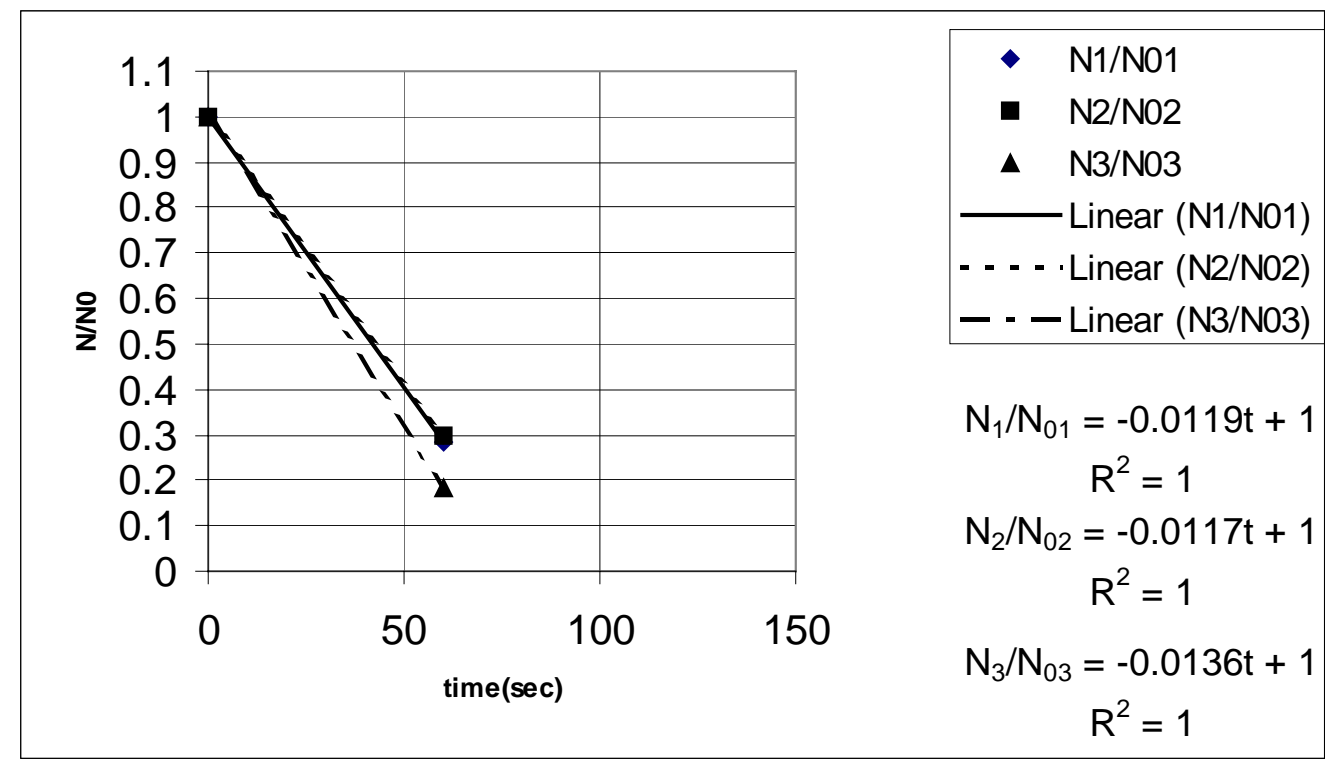

Figure C3. For PEO concentration $=200 \mathrm{mg} / \mathrm{l}$, relative particle count versus time of shearing at (a) $20 \mathrm{~s}^{-1}$ (b) $80 \mathrm{~s}^{-1}$ and (c) $190 \mathrm{~s}^{-1}$. Solid content $=0.2 \% \mathrm{v} / \mathrm{v}$, PEO MW $=1 \times$ $10^{6}$ 




(a)



(b)

Figure C4. Relative particle count versus time for PEO MW (a) $5 \times 10^{6}$, (b) $2 \times 10^{5}$. Solid content $=0.2 \%$, Shear rate $=80 \mathrm{~s}^{-1}$, PEO conc. $-100 \mathrm{mg} / \mathrm{l}$. 